\title{
Stellar population gradients in galaxy discs from the CALIFA survey ${ }^{\star}$
}

\section{The influence of bars}

P. Sánchez-Blázquez ${ }^{1}$, F. F. Rosales-Ortega ${ }^{1,2}$, J. Méndez-Abreu ${ }^{3}$, I. Pérez ${ }^{4}$, S. F. Sánchez ${ }^{5,6,7}$, S. Zibetti ${ }^{8}$, J. A. L. Aguerri ${ }^{9}$, J. Bland-Hawthorn ${ }^{10}$, C. Catalán-Torrecilla ${ }^{11}$, R. Cid Fernandes ${ }^{12}$, A. de Amorim ${ }^{7}$, A. de Lorenzo-Caceres ${ }^{3}$, J. Falcón-Barroso ${ }^{9}$, A. Galazzi ${ }^{8}$, R. García Benito ${ }^{7}$, A. Gil de Paz ${ }^{11}$, R. González Delgado ${ }^{7}$, B. Husemann ${ }^{7}$, Jorge Iglesias-Páramo ${ }^{5}$, B. Jungwiert ${ }^{13}$, R. A. Marino ${ }^{11}$, I. Márquez ${ }^{7}$, D. Mast ${ }^{14}$, M. A. Mendoza ${ }^{7}$, M. Mollá ${ }^{15}$, P. Papaderos ${ }^{16}$, T. Ruiz-Lara ${ }^{4}$, G. van de Ven ${ }^{17}$, C. J. Walcher ${ }^{18}$, and L. Wisotzki ${ }^{18}$

1 Departamento de Física Teórica, Universidad Autónoma de Madrid, 28049 Cantoblanco, Spain e-mail: p.sanchezblazquez@uam.es

2 Instituto Nacional de Astrofísica, Óptica y Electrónica, 72840 Tonantzintla, Puebla, México

3 School of Physics \& Astronomy, University of St Andrews, UK

${ }^{4}$ Departamento de Física y del Cosmos, Universidad de Granada, 18071 Granada, Spain

5 Centro Astronómico Hispano Alemán de Calar Alto (CSIC-MPIA), 4004 Almería, Spain

6 Instituto de Astronomía,Universidad Nacional Autonóma de Mexico, A.P. 70-264, 04510 Mexico DF, Mexico

7 Istituto de Astrofísica de Andalucía (IAA-CSIC), 18080 Granada, Spain

8 INAF-Osservatorio Astrofisico di Arcetri - Largo Enrico Fermi 5, 50125 Firenze, Italy

9 Instituto de Astrofísica de Canarias, 38205 La Laguna, Spain

10 Institute of Astronomy, School of Physics, University of Sydney, Sydney, NSW 2006, Australia

11 Departamento de Astrofísica y Ciencias de la Atmósfera, Universidad Complutense de Madrid, 28040 Madrid, Spain

12 Departamento de Física, Universidade Federal de Santa Catarina, PO Box 476, 88040-900, Florianópolis, SC, Brazil

13 Astronomical Institute, Academy of Science of the Czech Republic, Bocní 1401/1a, 14100 Prague, Czech Republic

14 Instituto de Cosmologia, Relatividade e Astrofísica ICRA, Centro Brasileiro de Pesquisas Físicas, Rua Dr.Xavier Sigaud 150, CEP 22290-180 Rio de Janeiro, RJ, Brazil

15 Departamento de Investigación Básica, CIEMAT, Avd Complutense 40, 28040 Madrid, Spain

16 Centro de Astrofísica da Universidade do Porto, Rua das Estrelas, 4150-762 Porto, Portugal

17 Max-Planck-Institut für Astronomie, 69117 Heidelberg, Germany

18 Leibniz-Institut für Astrophysik Potsdam (AIP), An der Sternwarte 16, 14482 Postdam, Germany

Received 13 February 2014 / Accepted 25 June 2014

\section{ABSTRACT}

While studies of gas-phase metallicity gradients in disc galaxies are common, very little has been done towards the acquisition of stellar abundance gradients in the same regions. We present here a comparative study of the stellar metallicity and age distributions in a sample of 62 nearly face-on, spiral galaxies with and without bars, using data from the CALIFA survey. We measure the slopes of the gradients and study their relation with other properties of the galaxies. We find that the mean stellar age and metallicity gradients in the disc are shallow and negative. Furthermore, when normalized to the effective radius of the disc, the slope of the stellar population gradients does not correlate with the mass or with the morphological type of the galaxies. In contrast to this, the values of both age and metallicity at $\sim 2.5$ scale lengths correlate with the central velocity dispersion in a similar manner to the central values of the bulges, although bulges show, on average, older ages and higher metallicities than the discs. One of the goals of the present paper is to test the theoretical prediction that non-linear coupling between the bar and the spiral arms is an efficient mechanism for producing radial migrations across significant distances within discs. The process of radial migration should flatten the stellar metallicity gradient with time and, therefore, we would expect flatter stellar metallicity gradients in barred galaxies. However, we do not find any difference in the metallicity or age gradients between galaxies with and without bars. We discuss possible scenarios that can lead to this lack of difference.

Key words. galaxies: abundances - galaxies: evolution - galaxies: formation - galaxies: spiral - galaxies: stellar content

\section{Introduction}

Spatially resolved stellar population studies in the disc region of spiral galaxies are sparse, with the exception of our own galaxy (see Freeman \& Bland-Hawthorn 2002, for a review;

* Tables 1-3 and Appendices are available in electronic form at http: //WWW . aanda.org
Friel et al. 2002; Yong et al. 2006; Carraro et al. 2007) and for some nearby galaxies, such as M33 (Monteverde et al. 1997; Barker et al. 2007; Williams et al. 2009a,b; Cioni 2009), M100 (Beauchamp \& Hardy 1997), M81 (Hughes et al. 1994; Davidge 2006a), NGC 2403 (Davidge 2007), NGC 300 (Vlajić et al. 2009; Kudritzki et al. 2008; Urbaneja et al. 2005; Gogarten et al. 2010) or M31 (Worthey et al. 2005). For disc galaxies outside the Local Group, stellar population gradients have mainly 
been investigated using colours (e.g., de Jong 1996; Peletier \& Balcells 1996; Jansen et al. 2000; Bell \& de Jong 2000; MacArthur et al. 2004; Taylor et al. 2005; Muñoz-Mateos et al. 2007, 2009; Prochaska et al. 2010). These studies find that disc galaxies tend to be bluer in the external parts. This trend has been interpreted as the consequence of a population that is older and more metal rich in the centre than in the external parts. However, there are large discrepancies in the magnitude of the stellar population gradients derived by different authors. This is because it is extremely difficult to disentangle the effects of age, metallicity and dust extinction (on average $\sim 1$ mag in the central regions, Ganda et al. 2009) using only colours. Spectroscopic studies may help alleviate the associated degeneracies, but the low surface brightness of the disc region and the nebular emission lines filling some of the most important age-diagnostic absorption lines make the analysis very difficult. This is why studies of stellar populations in disc galaxies using spectroscopy are scarce, and we are still lacking large enough samples to make statistical statements about the variation in age and metallicity as a function of other parameters. Some pioneering works tried to overcome the difficulty of measuring low-surface brightness absorption lines in the disc using narrow-band imaging or performing Fabry-Pérot interferometry with tunable filters (Beauchamp \& Hardy 1997; Molla et al. 1999; Ryder et al. 2005). Unfortunately, the low spectral resolution and poor signal-to-noise ratio $(\mathrm{S} / \mathrm{N})$ of the data compromised the results in each case. Furthermore, the works were restricted to the study of just a few indices (Mgb, Fe5207, Fe5335), which limited their ability to break the agemetallicity degeneracy. More recently, MacArthur et al. (2009) and Sánchez-Blázquez et al. (2011) have measured star formation histories as a function of radius using long-slit spectroscopy. However, long-slit data limit the number of spectra to add on the external parts and, therefore, the $\mathrm{S} / \mathrm{N}$. As a consequence, these analysis concentrated in the study of the bulges and inner discs. Both studies find very mild gradients in both age and metallicity in the disc region.

In the past years, a series of papers have been published using 2D data from the VENGA (Blanc et al. 2010), the PINGS (Rosales-Ortega et al. 2010) and the CALIFA (Sánchez et al. 2012a,b) surveys where the star formation history of a sample of nearby galaxies of all morphological types has been studied in detail (Yoachim et al. 2010, 2012; Sánchez-Blázquez et al. 2014; Pérez et al. 2013; González Delgado et al. 2014; Cid Fernandes et al. 2013, 2014). These papers show the potential of these data to overcome previous difficulties in the derivation of stellar population properties. In the present paper, we want to concentrate on the spatially resolved properties of the disc in spiral galaxies. In particular, we want to test the predictions that bars are very efficient agents in producing radial movements of stars across the disc without heating the disc (Minchev \& Famaey 2010).

The idea that stars in galactic discs may migrate radially across significant distances has received a lot of attention in the literature over the past few years (Sellwood \& Binney 2002; Roškar et al. 2008a,b; Berentzen et al. 2007; Minchev \& Famaey 2010; Minchev et al. 2011, 2012a,b, 2014a,b; Grand et al. 2012a,b; Comparetta \& Quillen 2012; Di Matteo et al. 2013; Brunetti et al. 2011; Shevchenko 2011; Kubryk et al. 2013). The reason is the possible important consequences that this process may have in a number of studies as the solar neighbourhood agemetallicity relation, the metallicity distribution function, the evolution of the metallicity gradient, the composition of the thick disc, and the reconstruction of star formation histories from current observations of stellar populations (Roškar et al. 2008b; Schönrich \& Binney 2009). These observations are used to constrain, among others, the amount of inflow and the role of feedback in chemical evolution models. Therefore, if we aim to understand the physics of disc formation, it is essential to understand the importance of stellar radial migrations.

The idea of radial migration was already suggested by Wielen (1977), to explain the metallicity of the Sun with respect to other stars of the same age in our solar neighbourhood. Because of a general increase in the non-circular motion with age, older stars oscillate more than young ones. However, for observationally constrained velocity dispersions, variations in galactocentric radius of at most few $\mathrm{kpc}$ are expected. A new mechanism was proposed by Sellwood \& Binney (2002) that can induce strong radial movements without significantly heating the disc due to the exchange of angular momentum at the corotation resonance (CR) of spiral arms. This mechanism can produce radial movements on the stars of several kpc in a few hundred Myr whilst keeping them in circular orbits. Because this mechanism involves CR, the spiral arms have to be transient or, otherwise, stars would get trapped in horseshoe orbits (Sellwood \& Binney 2002).

On the other hand Minchev \& Famaey (2010; see also Minchev et al. 2011, 2012a,b) proposed that spiral structure interacting with a central bar could be an extremely efficient mechanism for radial migration in galactic discs. Although angular momentum changes are more important at the vicinity of the corotation radius of each individual perturber, the non-linear coupling between the bar and spiral waves (e.g., Tagger et al. 1987; Sygnet et al. 1988) can make this mechanism effective over the entire galactic disc. Furthermore, being non-linear, this way of mixing can be significantly more efficient at increasing the angular momentum than transient spirals alone and works with both short- and long-lived spirals. In fact, Comparetta \& Quillen (2012) showed that, even if patterns are long-lived, radial migration can result from short-lived density peaks arising from interference among density waves overlapping in radius.

Bars are very common structures; the most recent optical studies indicate that approximately half of all massive and nearby discs galaxies contain bars (Barazza et al. 2008; Aguerri et al. 2009). This fraction increases in dust-penetrating nearinfrared wavelengths (Eskridge et al. 2000). The mass of the galaxy seem to be the main physical parameter regulating the bar fraction (e.g., Méndez-Abreu et al. 2010, 2012; Nair \& Abraham 2010). Given the fact that bars are very common and that our own Galaxy hosts a bar, it is extremely important to understand the influence of this structure in shaping the disc.

One of the predicted observational consequences of stellar radial migrations in the disc is a flattening of the chemical abundance profiles of the stellar component (e.g., Friedli et al. 1994; Friedli 1998; Roškar et al. 2008b; Loebman et al. 2011; Brunetti et al. 2011; Di Matteo et al. 2013), especially for those of the old stars (although this depend of the velocity dispersion of the stars when they were formed as stellar migration is less efficient for dynamically heated populations - see Minchev et al. 2014a,b for a discussion). Therefore, a way to test the importance of the mechanism proposed by Minchev \& Famaey (2010) is to compare the metallicity profiles of galaxies with and without bars.

To do this, in this paper, we make a comparison of the age and stellar metallicity gradients in a sample of low-inclination spiral galaxies with and without bars from the CALIFA survey (Sánchez et al. 2012a,b). Section 2 presents the sample, Sect. 3 the derivation of the star formation histories, mean ages and metallicities and Sect. 4 our results. In Sect. 5 we analyse the metallicity gradients for stellar populations of different ages and Sect. 6 shows briefly the relation between the stellar populations 
in the disc and the bulge. In Sect. 7 we discuss the possible scenarios that can explain our results whilst Sect. 8 presents our conclusions.

\section{Sample and data reduction}

The data for the present study are taken from the Calar Alto Legacy Integral Field Area survey (CALIFA; Sánchez et al. $2012 \mathrm{a}, \mathrm{b})$. The survey is observing a statistically well defined sample of $\sim 600$ galaxies in the local Universe using 250 observing nights with PMAS/PPAK integral field spectrophotometer mounted at the Calar Alto $3.5 \mathrm{~m}$ telescope. The targets are randomly selected from the mother sample that comprises 939 galaxies from the SDSS DR7 (Abazajian et al. 2009). The main selection criteria are the angular isophotal size $\left(45^{\prime \prime}<\right.$ $D_{25}<80^{\prime \prime}$, where $D_{25}$ is the isophotal diameter in the SDSS $r$-band) and the proximity of the galaxies $(0.005<z<0.03)$. PPAK offers a combination of extremely wide field of view $\left(>1 \operatorname{arcmin}^{2}\right)$ with a high filling factor in one single pointing $(65 \%)$. A dithering scheme with three pointing has been adopted in CALIFA in order to cover the complete field-of-view of the central bundle and to increase the spatial resolution of the data. The spectra cover the range 3400-7300 $\AA$ in two overlapping setups, one in the red (3745-7300 $\AA$ ) at a spectral resolution of $R=850$ (V500 setup) and one in the blue (3400-4750 $\AA$ ) at $R \sim 1650$ (V1200 setup). In the present paper we have combined the two setups by degrading the spectral resolution of the blue part of the spectra to match the resolution of the red using a wavelength-dependent Gaussian smoothing kernel. The exposure time is fixed for all the observed objects. For the V500 setup a single exposure of $900 \mathrm{~s}$ per pointing of the dithering scheme is taken whilst for the V1200 setup 3 or 2 exposures of $600 \mathrm{~s}$ or $900 \mathrm{~s}$, respectively, are obtained per pointing. The data reduction is explained in detail in Sánchez et al. (2012a) and Husemann et al. (2013). The basic reduction tasks include cosmic ray rejection, optimal extraction, flexure correction, wavelength and flux calibration and sky subtraction. Finally, all three pointings are combined using a flux conserving inverse distance weighting scheme (see the original paper for details), to reconstruct the final data cube, with a one arcsec spatial sampling.

The selection criteria to build the sample (diameter and redshift) are such that the selected objects represent a wide range of galactic properties such as morphological types, luminosities, stellar masses and colours. Further details on the selection criteria effects and a detailed characterization on the CALIFA mother sample are explained in Sánchez et al. (2012a,b).

For this study, we selected galaxies that are morphologically classified as discs, without signs of recent interactions and with inclination lower than 60 degrees. The morphological classification will be presented in Walcher et al. (in prep.), and is based on an eyeball classification by 5 members of the collaboration using $r$ and $i$ band SDSS images. The constrain in inclination is given by the difficulty in detecting the bar presence in highly inclined galaxies. This restrictions lead us with a sample of 62 galaxies, 28 of which are unbarred, 25 strongly barred (B) and 9 weakly barred (AB). Although the presence of the bar is determined visually we also performed an ellipse analysis to calculate its size and strength (Méndez-Abreu et al., in prep.). The strength of the bar, which measure the contribution of the bar to the total galaxy potential, is derived using:

$f_{\mathrm{bar}}=\frac{2}{\pi}\left(\arctan \left(1-\epsilon_{\mathrm{bar}}\right)^{-1 / 2}-\arctan \left(1-\epsilon_{\mathrm{bar}}\right)^{+1 / 2}\right)$ where $\epsilon_{\mathrm{bar}}$ is the intrinsic mean ellipticity in the bar region. This parameter was defined by Abraham \& Merrifield (2000) and it is correlated with the most widely used parameter $Q_{g}$, defined by Buta \& Block (2001; Laurikainen et al. 2007). The size of the bar has been calculated as the mean value of those determined with three different methods: (i) the maximum of the ellipticity; (ii) the minimum of the ellipticity and the (iii) change in the position angle of the ellipses in more than 5 degrees (see Méndez-Abreu et al. 2014, for details).

In the present study we also use the stellar mass determined by fitting SED models to galaxy photometry with Kcorrect (Bekeiraite et al., in prep.). These masses agree extremely well with the stellar masses derived with full spectral fitting using STARLIGHT (González Delgado et al. 2014). We will also use the effective radius of the disc (radius enclosing half of the light of the disc component, i.e., excluding the bulge, $r_{\text {eff }}$ hereafter). This radius is calculated using an analysis of the azimuthal surface brightness profile derived through an isophotal analysis of the SDSS imaging survey g-band images (York et al. 2000). In the region dominated by the disc, the profile is fitted with a pure exponential profile, using the classical formulae:

$I=I_{0} \exp \left[-\left(r / r_{\mathrm{d}}\right)\right]$.

The effective radius of the disc is related to the scale-length through $r_{\mathrm{eff}}=1.67835 r_{\mathrm{d}}$ and is very well correlated with the total effective radius obtained with the curve of growth (see Appendix A of Sánchez et al. 2013b, for more details about how the disc radius is determined). Table 5 show the main properties of our final sample.

\section{Analysis}

We spatially bin by means of the centroidal Voronoi tessellation algorithm of Cappellari \& Copin (2003) to ensure a minimum $\mathrm{S} / \mathrm{N}$ of 40 (per $\AA$ ) at $5800 \AA$, necessary for a reliable determination of the stellar population properties ${ }^{1}$. Pre-processing steps include spatial masking of foreground/background sources, very low $\mathrm{S} / \mathrm{N}$ spaxels and bad pixels.

\subsection{Emission line cleaning, and determination of radial velocity and line broadening}

On the binned spectra, we run the code GANDALF (Sarzi et al. 2006). GANDALF fits, simultaneously, the absorption and emission lines, treating the latter as additional Gaussians. In a first step, emission lines are masked and the absorption line spectrum is fitted with the penalised pixel-fitting pPXF (Cappellari \& Emsellem 2004), using as templates the stellar population models of Vazdekis et al. (2010, V10 hereafter) based on the MILES stellar library (Sánchez-Blázquez et al. 2006; Cenarro et al. 2007; Falcón-Barroso et al. 2011) ${ }^{2}$. In this step, radial velocities and absorption line broadening ${ }^{3}$ ( $\sigma$, hereafter) for the stellar component are derived. The best values of velocity and $\sigma$ and the best template mix are then used as initial values for the calculation of emission lines using GANDALF. Emission lines equivalent

\footnotetext{
1 The same analysis has been repeated for azimuthally binned spectra and the results remain unaltered.

2 The stellar population models and the stellar library are publicly available at http://miles.iac.es.

3 The velocity dispersions for these galaxies are calculated using the data from the V1200 dataset (Falcón-Barroso et al., in prep.). What we derive here is the total broadening of the lines, including the instrumental and the doppler broadening.
} 

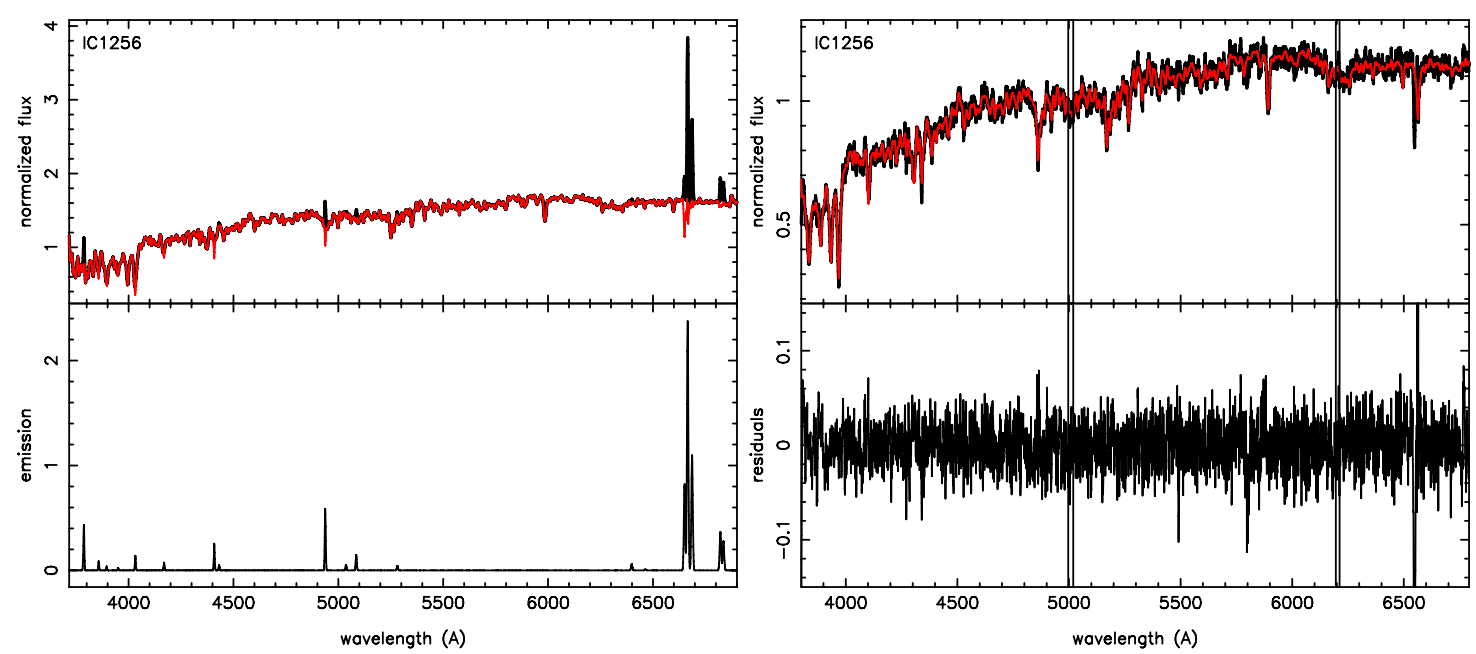

Fig. 1. Left panel - top panel: original observed central spectrum (central spaxel) of IC 1256 (black line) and the one corrected from emission using GANDALF (red line). Bottom panel: emission line spectrum. Right panel - top panel: fit to the central spectra of the galaxies from the sample (red line). Bottom panel: residuals from the fit. Masked areas are indicated with vertical lines.

widths, radial velocities and $\sigma$ for the gaseous component are derived in this second step. The fit allows for low-order Legendre polynomial in order to account for small differences in the continuum shape between the pixel spectra and the templates. The best fitting template mix is determined by a $\chi^{2}$ minimization in pixel space. Emission lines were subtracted from the observed spectra. Figure 1 shows, as an example, the spectrum of the central spaxel for the galaxy IC 1256, before and after subtracting the emission lines.

\subsection{Star formation histories}

Disc galaxies certainly show complex star formation histories. In the last decade, numerical techniques have been developed to derive the whole evolution of the star formation history with time using as much information as possible from the spectra (Heavens et al. 2000; Panter et al. 2003; Cid Fernandes et al. 2005; Ocvirk et al. 2006a,b; Tojeiro et al. 2007; Koleva et al. 2009 - see, e.g., Walcher et al. 2011; Sánchez et al. 2012a,b; Rosales-Ortega et al. 2012).

To derive star formation histories we used the code STECKMAP (STEllar Content and Kinematics via Maximum A Posteriori likelihood, Ocvirk et al. 2006a,b) on the emission linecleaned spectra (see previous section). STECKMAP projects the observed spectrum onto a temporal sequence of models of single stellar populations to determine the linear combination that fits the observed spectrum best. The weights of the various components of this linear combination indicate the stellar content of the population. As templates, we use the stellar population models by V10 based on the MILES library (Sánchez-Blázquez et al. 2006). We chose those models with a Kroupa Universal IMF (Kroupa 2001) ${ }^{4}$ and a range of ages and metallicities from $63 \mathrm{Myr}$ to $17.8 \mathrm{Gyr}$ and $-2.32<[\mathrm{Z} / \mathrm{H}]<+0.2$ respectively.

STECKMAP is a Bayesian method that simultaneously recovers the kinematic and stellar population properties via a maximum a posteriori algorithm. It has been extensively tested

\footnotetext{
4 This IMF is a multi-part power-law IMF, which is similar to the Salpeter (1955) IMF for stars of masses above $0.5 M_{\odot}$, but with a decreasing contribution of lower masses by means of two flatter segments.
}

and used in a variety of applications ${ }^{5}$. The method is not parametric and does not make any a priori assumption regarding the shape of the star formation history. The only condition that STECKMAP imposes is that the different unknowns, namely the stellar age distribution, the age-metallicity relation and the lineof-sight velocity distributions or the broadening function have to be smooth in order to avoid extreme oscillating solutions that are not robust and most likely unphysical. The function to minimise is defined as:

$$
Q_{\mu}=\chi^{2}(s(x, Z, g))+P_{\mu}(x, Z, g),
$$

which is a penalised $\chi^{2}$, where $s$ is the modelled spectrum resulting from the age distribution $x$, the age-metallicity relation $(Z)$ and the broadening function $(g)^{6}$. The penalization $P_{\mu}$ can be written as: $P_{\mu}(x, Z, g)=\mu_{x} P(x)+\mu_{Z} P(Z)+\mu_{v} P(g)$, where the function $P$ gives high values for solutions with strong oscillations (i.e., a rapid variation of the metallicity with age or a noisy broadening function) and small values for smoothly varying solutions. Adding the penalization $P$ to the function $Q$ is exactly equivalent to inject a priori information into the problem. In practice, this is like imposing an a priori probability density to the solution as $f_{\text {prior }}(x)=\exp \left(-\mu_{x} P(x)\right)$. For this work we define $P$ as a quadratic function of the unknown $x$, involving a kernel $L$. We use a Laplacian smoothing kernel of the age distribution and a gradient kernel for the age-metallicity relation, as in Ocvirk (2010; see Ocvirk et al. 2006a, for details). Choosing the right values of the smoothing parameters $\mu_{x, Z, v}$ is not a trivial problem. In principle, one could choose the values giving the smaller $\chi^{2}$ in the fit, but this usually yields a wide range of smoothing parameters, spanning typically $3-4$ decades, in which the fit is acceptable. In any case, although the detailed shape of the derived star formation history can be affected by this choice, the range of smoothing parameters we are using neither change the overall interpretation of the star formation history nor the mean age and metallicity values, which is what are are going to use across the paper. We are using in this work, $\mu_{x}=1$ and $\mu_{z}=1$.

\footnotetext{
5 It is a public tool and can be obtained at http://astro. u-strasbg.fr/ ocvirk/

6 Note that STECKMAP fits different broadening function for different ages as different populations are expected to have different velocity dispersions (e.g., House et al. 2011).
} 
NGC2253

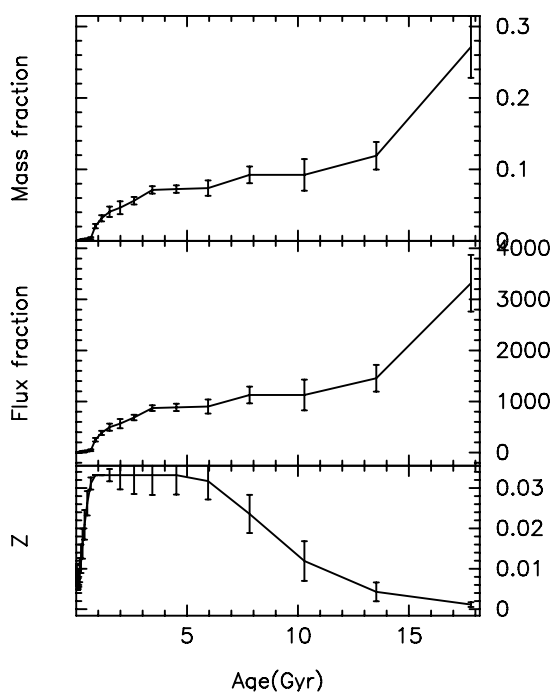

Fig. 2. Typical output of STECKMAP for the central spectrum of NGC 2253. Top panel: mass fraction of stars of different ages; middle panel: flux fraction; bottom panel: evolution of metallicity $Z$. The error bars represent the root-mean-square dispersion from the mean, of a series of 250 Monte Carlo simulations where each pixel of the spectrum is perturbed with noise following a Gaussian distribution of width given by the error spectrum.

In the present analysis we have not fitted simultaneously the star formation histories and the kinematics. Instead, for the kinematic we adopt the solution of pPXF that we obtained in the correction from emission line (see Sect. 3.1). The reasons are explained in detail in Appendix B of Sánchez-Blázquez et al. (2011). Basically, the existing degeneracy between the metallicity and the velocity dispersion (Koleva et al. 2008) biases the mean-weighted metallicites if both parameters are fitted at the same time. One important detail of this work is that we do not use the continuum in our derivation of the star formation history. This is to avoid spurious results due to possible flux calibration errors or extinction. To do this, we multiply the model by a smooth non-parametric transmission curve, representing the instrumental response multiplied by the interstellar extinction. This curve has 30 nodes, spread uniformly along the wavelength range, and the transmission curve is obtained by spline interpolating between the nodes. The latter are treated as additional parameters and adjusted during the minimization procedure. By using this curve to remove the continuum, we do not have to correct from extinction, as dust extinction does not change the equivalent width of the absorption lines (MacArthur 2005). We fit the whole wavelength range of the data, masking the regions affected by sky residuals or others defects of the detectors. Figure 1 shows the integrated spectrum of IC 1256 together with the best fit obtained with STECKMAP.

The typical STECKMAP outputs give the proportion of stars at each age that are contributing to the observed flux and to the stellar mass and the evolution of the metallicity with time. Figure 2 shows an example of these outputs for the central spectrum of NGC 6155. It is easy to see from these outputs that our results imply that $20-30 \%$ of the stars have ages older than the most recent estimates of the age of the Universe. This is a well known problem in the stellar population models (e.g., Vazdekis et al. 2001; Schiavon et al. 2002; Maraston et al. 2011, V10). Several possible solutions have been proposed to alleviate this so-called zero point problem, and, in fact, several groups have successfully reconcile the age of globular clusters with $\Lambda \mathrm{CDM}$ cosmology (see Krauss \& Chaboyer 2003); Percival \& Salaris (2009) showed that systematic uncertainties associated with the three fundamental stellar atmospheric parameters might have a non negligible impact on the resulting SSP line-strengths. In particular, a relatively small offset in the effective temperature of $50-100 \mathrm{~K}$, which is of the order of the systematic errors in the conversion from temperature to colours used in the V10 models used here (Alonso et al. 1996) may change the age of a $14 \mathrm{Gyr}$ stellar population by 2-3 Gyr and alleviate the zero point problem (see V10 for more details). Isochrones that have into account effects such as $\alpha$-enhancement and diffusion of heavy elements also help to alleviate the problem (Vazdekis et al. 2001). Different ages are also obtained when using isochrones with different opacities (e.g., Bruzual \& Charlot 2003). For example, the use of Padova (Girardi et al. 2000; which are those used by V10) gives older ages than Padova (Bertelli et al. 1994). Schiavon et al. (2002) also stressed the importance of correctly modelling the luminosity function at the level of the giant branch for the particular problem of globular clusters. He showed, for 47 Tuc, that if the observed luminosity function is used rather than the theoretical ones, the spectroscopic and the colour-magnitude diagram ages coincide and are lower than the age of the Universe.

However, despite these possibilities, there is no physical reason to prefer Padova (1994) to Padova (2000), which include improved physics. There is also no reason to chose a different temperature scale than the one we chose in our models to transform from the theoretical to the observational plane. On the other hand, we cannot determine, observationally, the luminosity function of red giant branch stars in our galaxies. Our models make use of the latest and, in our opinion, best possible ingredients, despite this choice leads to very old ages. We prefer to use the whole range of model (i.e., we use SSPs with ages older than the age of the Universe) as, otherwise we could be biasing the results artificially. In any case, the present study is comparative and so the age scale should not affect our main conclusions. We can obtain a mean $\log ($ age $)$ and metallicity weighting with the light or with the mass of each population as:

$$
\begin{aligned}
\langle\log q\rangle_{\mathrm{MW}} & =\frac{\sum_{i} \operatorname{mass}(i) \log q_{i}}{\sum_{i} \operatorname{mass}(i)}, \\
\langle\log q\rangle_{\mathrm{LW}} & =\frac{\sum_{i} \operatorname{flux}(i) \log q_{i}}{\sum_{i} \operatorname{flux}(i)}
\end{aligned}
$$

where $q$ is the physical parameter we want to estimate, i.e., age or metallicity, and mass $(i)$ and flux $(i)$ are, respectively, the reconstructed mass and flux contributions of the stars in the $i$ th age bin, as returned by STECKMAP. When present, young stars are very luminous in the optical range, therefore, they will contribute more to the light-weighted values. This means that the light-weighted values of age will be strongly biased towards the youngest stellar component ages. The mass weighted values will be less biased towards the age and metallicity of the youngest components however, they are also more uncertain. This is especially true when the contribution by mass of the old stars is high, as these stars are not very luminous and, therefore, their contribution to the observed spectrum is low. We have to note here that the average values of age and metallicities can change considerably if we add them logarithmically or linearly (Sánchez-Blázquez et al. 2011; González Delgado et al. 2014).

We have obtained maps of the mean $\log ($ age $)$ and metallicities weighting with both light and mass, for all the galaxies of our sample. Figure 3 shows an example for one galaxy of 

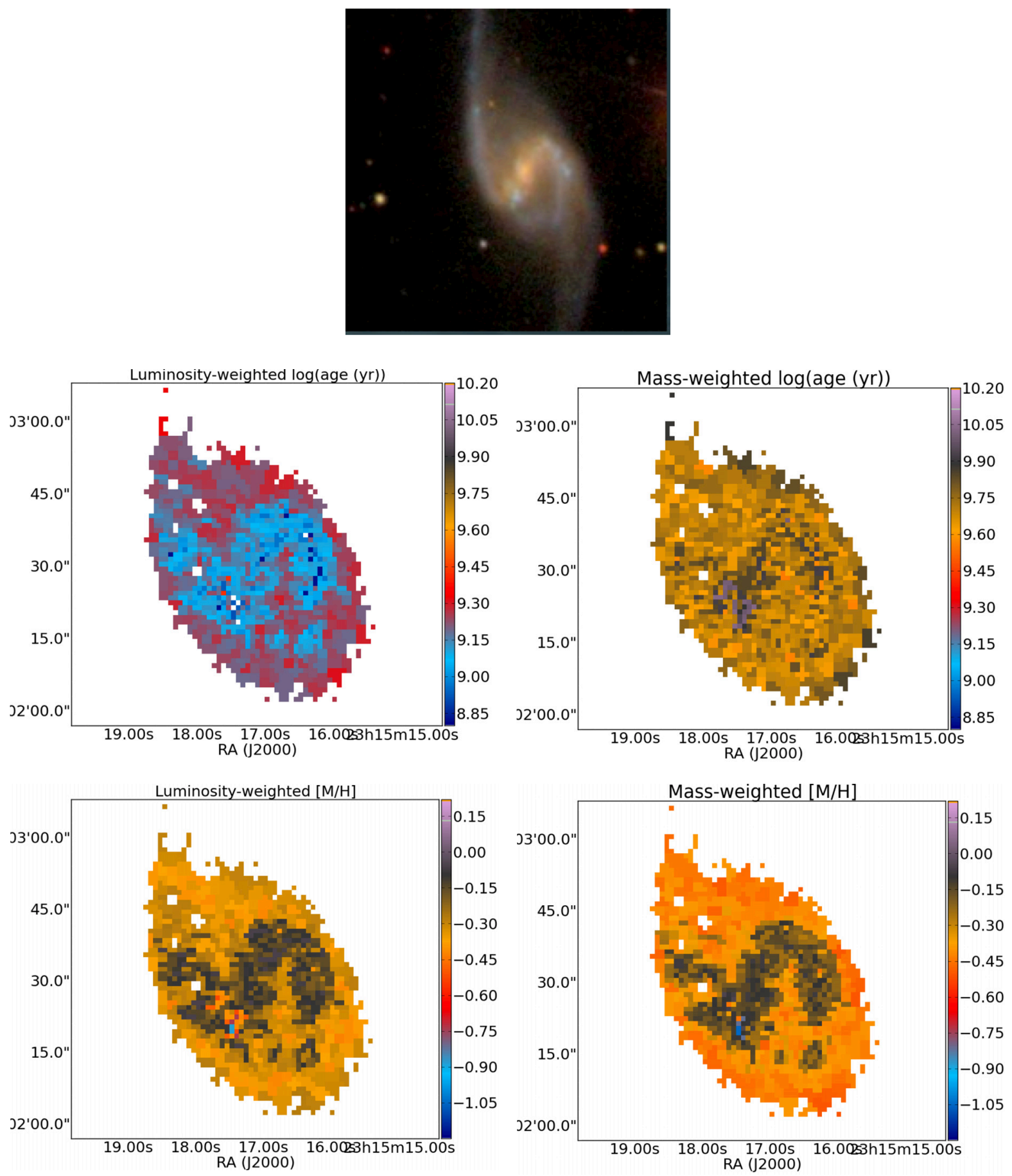

Fig. 3. Mean $\log ($ age $)$ and metallicity maps for one of the galaxies of our sample, NGC 7549. The maps for the rest of the sample are available in Appendix C.

our sample, NGC 7549. The rest of the maps are available in Appendix C.

Note that the detailed spatially resolved values for individual galaxies are method and model dependent. To check the extent of this dependence, we have compared our results with those obtained using STARLIGHT (Cid Fernandes et al. 2005) together with a set of models combination of V10 and González Delgado et al. (2005) for young stars (see Pérez et al. 2013 and González Delgado et al. 2014). While none of the results presented here depend on the method used, details on individual galaxies may change. A detailed description of the differences in the derived stellar population parameters using several methods and stellar population models will be presented in a future paper.

\section{Results}

\subsection{Stellar population gradients}

To visualise the variation of the stellar population trends with the radius of the galaxies, we average, from the Voronoi binned image, the values of age and metallicities along isophotes with 
the ellipticity and position angle of each galaxy (all the ellipses for a given galaxy with the same ellipticity and orientation).

Age gradients: Figs. B.1-B.3 show the variation of the mean age, weighted with both the mass and the light, for the sample of barred, unbarred and weakly barred galaxies respectively. The radius has been normalised to the effective radius of the disc.

In this paper we are only considering the gradients in the disc region. To obtain the radius where the disc light starts to dominate over the bulge, we analysed the one-dimensional surface brightness profiles, fitted the disc component with an exponential law and estimated the contribution of the bulge as the flux above the exponential fit. The $r_{\text {disc } 0}$ is defined as the one at which the surface brightness level coincides with that of the exponential component of the fit. We used the SDSS $r$-band imaging data, available for the whole sample.

The first thing that can be seen in the figures is that the mass-weighted age gradients are very flat and the mass-weighted mean ages are, in most cases, around $\sim 10 \mathrm{Gyr}$, even in the most external parts sampled by our data. This is in agreement with results from previous studies using long-slit spectroscopy (e.g., MacArthur et al. 2009; Sánchez-Blázquez et al. 2011), although these were based on a reduced number of galaxies ( 8 and 4 respectively) and did not reach radii as large as those in the present study. This result is also in agreement with studies of resolved stellar populations in nearby disc galaxies, which find that all galaxies host a high percentage of old stellar populations at all radii (Williams et al. 2009a,b; Gogarten et al. 2010; Barker et al. 2007), although we do not reach radii as large as those sampled in studies of resolved stellar populations.

Metallicity gradients: it has been well established that in a disc growing inside-out, metallicity decreases from the centre of the galaxy outwards (Goetz \& Koeppen 1992; Matteucci \& Francois 1989). Figures C.1-C.3 show the variation of the light- and mass-weighted metallicity gradients for our sample of barred, unbarred and weakly barred galaxies respectively. It can be seen that, in general, we find this decrease of metallicity with radius, although with some exceptions. The light-weighted metallicity (biased towards the values of the youngest stars) is always higher than the mass weighted value (which largely reflects the metallicity of the old stars), showing the metallicity evolution of the galaxy, where new stars formed from gas enriched by the previous stellar generations. The slopes of the gradients are, on the other hand, very similar for the mass- and light-weighted components. We will analyse in more detail the metallicity gradient in populations of different ages in Sect. 5.

\subsection{Stellar population gradients}

We quantify the variation of age and metallicity with radius in two different ways:

1. method 1: we perform a linear fit in the radial region where the surface brightness profile is dominated by the disc.

2. method 2: we measure the stellar parameters difference between $1.5 r_{\text {eff }}$ ( 2.5 scale lengths of the disc) and $r_{\text {disc } 0}$, which correspond the radius at which the light starts being dominated by the disc.

For barred galaxies, numerical simulations predict that the flattening of the metallicity gradients due to the non linear coupling of a bar and spiral arms is more prominent beyond the bar corotation, which causes variations in the slope of the gradient at this radius (e.g., Friedli 1998; Minchev et al. 2013; Di Matteo et al. 2013). Therefore, for the method 1 we use only those values at radii larger than the bar corotation. However, in the majority of the galaxies we do not see any variation of
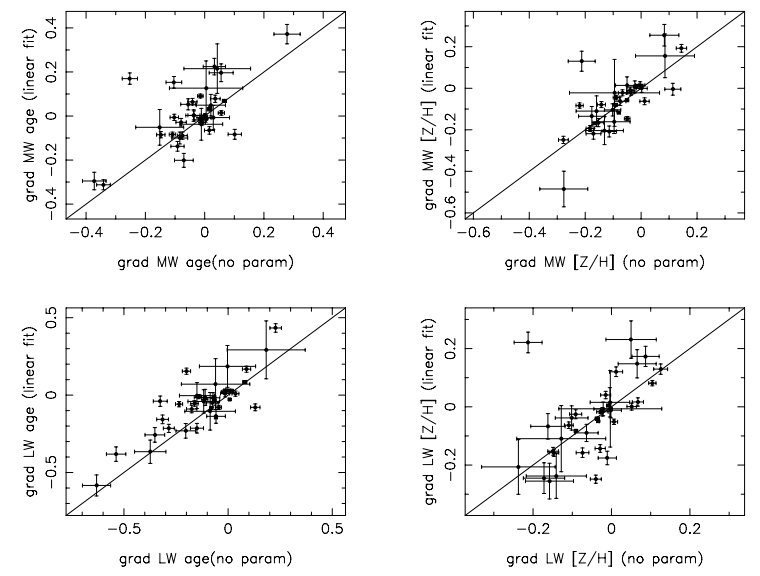

Fig. 4. Comparison of the stellar population gradients calculated with a linear fit ( $y$-axis) and calculated as the difference between the parameters (age or metallicity) at $1.5 r_{\text {eff }}$ and at $r_{\text {odisc }}$ (see text for details).

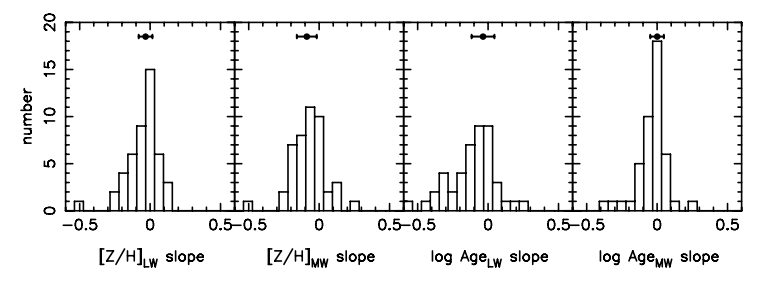

Fig. 5. Distribution of stellar population gradients. From left to right: luminosity-weighted metallicy, mass-weighted metallicity, luminosityweighted age and mass-weighted age. The dot with the error bars show the value of the mean and its error.

the slope at this radius and, therefore, we perform the linear fit in the whole disc region. Note that we have not calculated the corotation radius of the bars. However, recent results by Aguerri et al. (2013) - using the Tremaine \& Weinberg (1984) method to calculate bar pattern speed - show that the mean value of the ratio $R_{\mathrm{CR}} / R_{\text {bar }}$ is around 1 (where $R_{\mathrm{CR}}$ is the corotation radius and $R_{\mathrm{bar}}$ the length of the bar) and that there is not much dependence with the morphological type. Therefore, we will use the bar length as an orientative position of the bar corotation. Throughout the paper we designate the slope of the linear fits: $[Z / H]=a+b\left(r / r_{\text {eff }}\right)$ and $\log$ Age $=a+b\left(r / r_{\text {eff }}\right)$ with $\operatorname{grad}[Z / H]$ and grad Age respectively.

For method 2 we always measure the difference between the metallicity (and age) at $r_{\mathrm{disc} 0}$ and at $1.5 r_{\mathrm{eff}}$, independently of the presence or not of the bar.

Figure 4 shows the comparison of the gradients obtained with both methods. As can be seen, the values are compatible within the errors. Tables 6 and 7 list all the values calculated with method 1 and 2 respectively. In the second case we only consider those galaxies for which we reach $1.5 r_{\text {eff }}$. For the rest of the paper we will use the gradients calculated with the method 2, as they are more readily reproducible and do not assume any shape on the variation of the stellar parameters with radius.

Figure 5 shows the distribution of the stellar population gradients for the complete sample of galaxies. The mean age gradient for the whole sample is $-0.036 \pm 0.010$ and $0.000 \pm$ $0.006 \mathrm{dex} / r_{\mathrm{eff}}$ when averaging with the light and the mass respectively, whilst the mean light- and mass-weighted metallicity gradients are $-0.032 \pm 0.006 \mathrm{dex} / r_{\mathrm{eff}}$ and $-0.087 \pm 0.008 \mathrm{dex} / r_{\mathrm{eff}}$ respectively. We stress here that these values represent the 

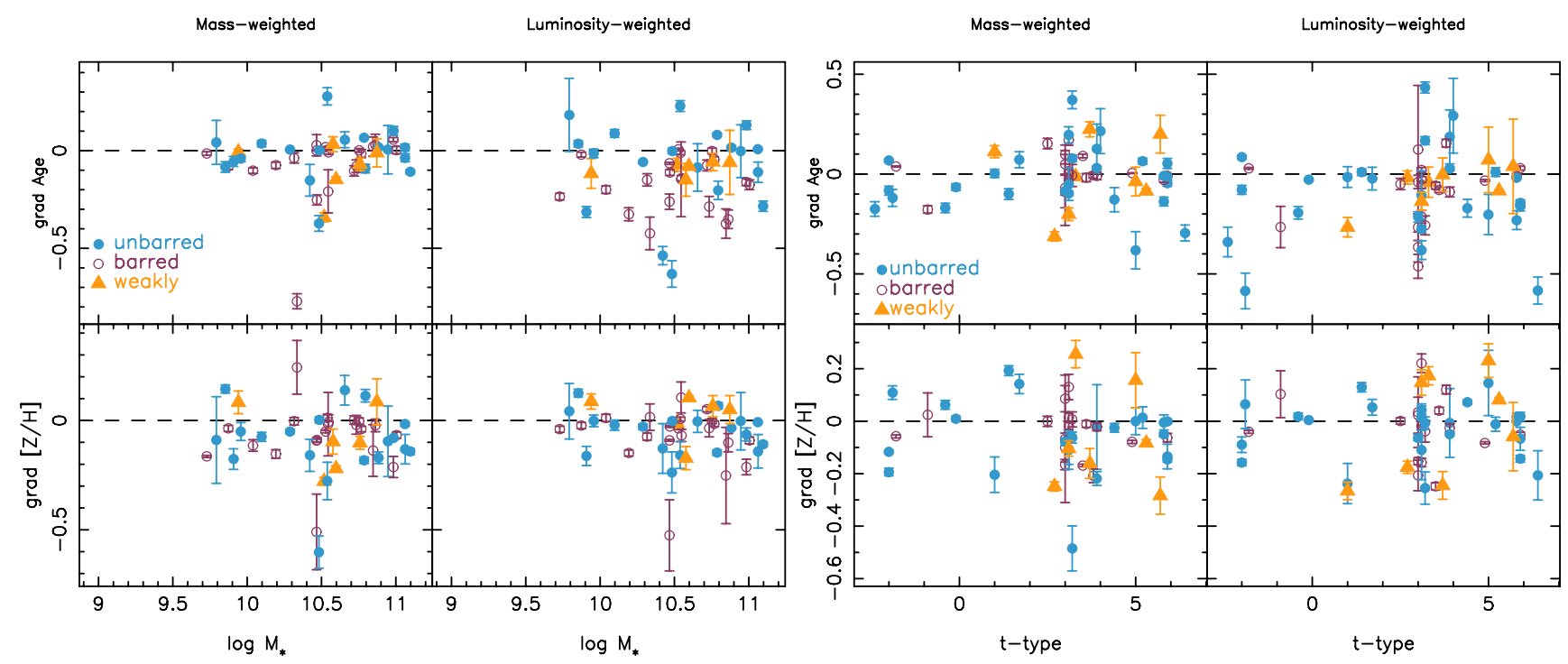

Fig. 6. Age (top panels) and metallicity (bottom panels) gradients as a function of the stellar mass and the t-type for our sample of barred (red) and unbarred (blue) galaxies. We have also represented weakly barred galaxies in orange. Both, mass- (left panels) and light-weighted (right panel) values are represented.

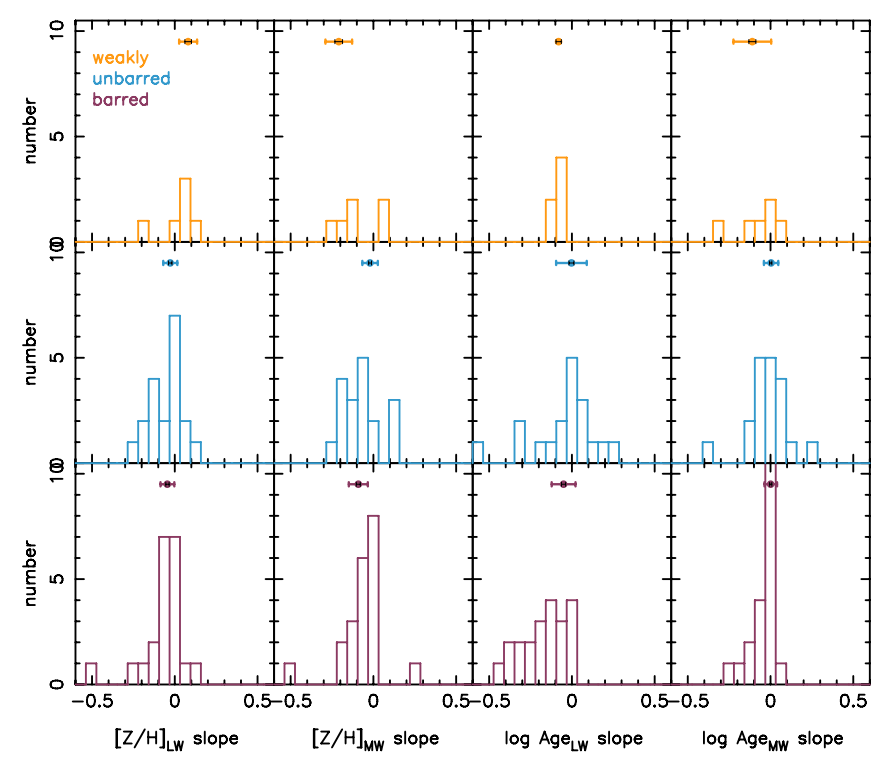

Fig. 7. Slope of the gradients of: luminosity-weighted [Z/H] (first column); mass-weighted metallicity (second column); luminosity-weighted age (3rd column) and mass-weighted age (4th column), for the different subsamples: weakly barred (top row); unbarred (middle row) and barred galaxies (bottom row). The point on the top of the diagram indicates the mean value (weighted with the errors) and the colored error bar the rms dispersion. The black error bar shows the dispersion expected by the errors

gradients in the disc regions, excluding the bulge. As can be seen, the gradients in both quantities are very shallow.

\subsection{Comparison between barred and unbarred galaxies}

The main goal of the present work is to quantify the influence of bars in shaping the metallicity gradient in disc galaxies. Figure 6 shows the metallicity gradients (in the disc region) as a function of the stellar mass and the morphological t-type.

As can be seen in the figure, contrary to the prediction of numerical simulations we do not find any difference in the values of the slope between the gradients of barred and unbarred galaxies. Table 4 lists the mean values of the gradients for the different subsamples. To visualise this in an easier way we plot, in Fig. 7, the histograms showing the slope of the gradients in the different parameters for the three subgroups (barred, unbarred and weakly barred galaxies). A t-test shows that the difference between the mean values of barred and unbarred galaxies are not significant at a $95 \%$ probability level. This is true for the age and the metallicity (both for the mass-weighted and the luminosityweighted values).

It is interesting to note, also, the lack of correlation between the slope of the gradients and the stellar mass or the t-type of the galaxies. There may be a weak correlation between the luminosity weighted metallicity gradient and the stellar mass, in the sense that more massive galaxies have a steeper gradient, but this correlation is not statistically significant. This lack of trends 
Table 4. Mean values for the gradient slope in the different stellar population parameters for barred and unbarred galaxies.

\begin{tabular}{lrlll}
\hline \hline Gradient & Mean & RMS & RMS $_{\text {exp }}$ & $N_{\text {gal }}$ \\
\hline MW age (barred) & 0.011 & 0.040 & 0.001 & 28 \\
MW age (unbarred) & -0.008 & 0.044 & 0.002 & 27 \\
\hline LW age (barred) & -0.017 & 0.059 & 0.002 & 28 \\
LW age (unbarred) & -0.051 & 0.103 & 0.002 & 27 \\
\hline MW [Z/H] (barred) & -0.092 & 0.067 & 0.002 & 25 \\
MW [Z/H] (unbarred) & -0.063 & 0.083 & 0.003 & 23 \\
\hline MW [Z/H] (barred) & -0.040 & 0.068 & 0.002 & 26 \\
MW [Z/H] (unbarred) & -0.013 & 0.043 & 0.002 & 25 \\
\hline
\end{tabular}

Notes. Third column indicate the root-mean-square dispersion among the mean values and forth column the dispersion expected by the measured errors in the gradients. Last column indicate the number of galaxies used to calculate these values.

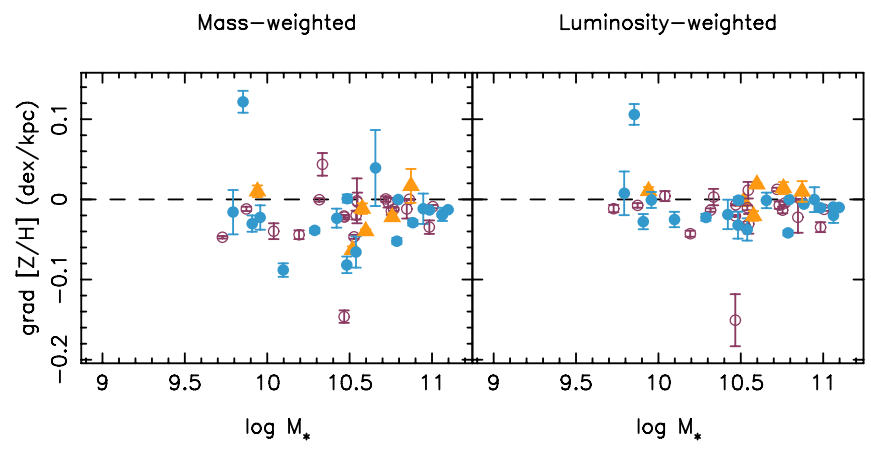

Fig. 8. Mass- (left) and luminosity-weighted (right) gradients vs. stellar mass. The slope of the gradient has been calculated in dex/kpc. The colours and the symbols are the same as in Fig. 6.

is similar to that found in the gas-phase metallicity (Diaz 1989; Sánchez et al. 2012b; Sánchez et al. 2014) when the metallicity gradient is normalised to a physical scale of the disc (e.g., the $R_{25}$ or the disc scale-length).

On the other hand, some studies have found a correlation between the gas-phase metallicity gradient (measured in physical scales, dex/kpc) and the morphological type or the mass, with early-type, and more massive spirals showing shallower slopes (e.g., McCall et al. 1985; Vila-Costas \& Edmunds 1992; Oey \& Kennicutt 1993). To see if a trend between the metallicity gradient and the mass is visible in our data when the gradient is measured in $\mathrm{dex} / \mathrm{kpc}$, we repeated the fits without normalizing to the effective radius of the disc and transforming the radius to kpc. The results are shown in Fig. 8. As can be seen, even in this case, we do not see clear trends between the stellar metallicity gradient and the mass.

Another prediction of numerical simulations is that the flattening of the metallicity gradient due to radial mixing is stronger for the old populations, as they have had more time to migrate along the disc. Because one of the STECKMAP outputs is the age-metallicity relation, we are, in principle, able to obtain a metallicity gradient for the old stars. We have obtained the mean metallicity for stars with ages $>6$ Gyr. This is what is shown in Fig. 9. The mean luminosity-weighted values of the metallicity gradients are $-0.04 \pm 0.06$ and $-0.06 \pm 0.07$ for unbarred and barred galaxies, respectively (the error represent the root mean square - rms - dispersion). A t-test comparing the mean values show, however, that the differences are not statistically significant.

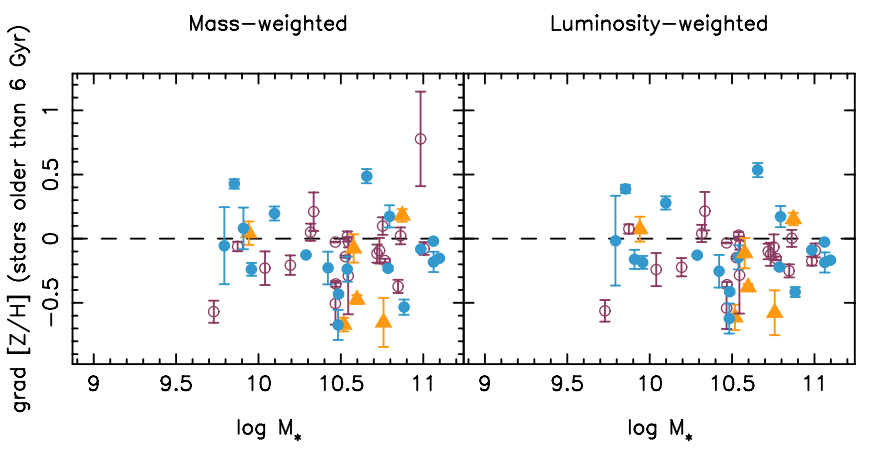

Fig. 9. Mass-(left panel) and luminosity-weighted (right panel) stellar metallicity gradient as a function of total stellar mass for stars older than 6 Gyr. The colours and the symbols are the same as in Fig. 6.

However, the expectation for a flatter metallicity gradient in old stars is based on predictions of idealised numerical simulations where the disc is evolved in isolation (e.g., Minchev et al. 2012a,b). Recently, it has been shown by Minchev et al. (2014a,b) - using zoom cosmological simulations of disc galaxies presented in Martig et al. (2012) - that the large vertical velocity dispersion of old stars (which were born hot at high redshift, or get heated early-on due to strong merger activity) prevent strong migration efficiency in this population (see also Brunetti et al. 2001). It worth noting, nevertheless, that the vertical velocity dispersion of the stars in cosmological and noncosmological simulations is still considerably larger than what is observed in our own Galaxy, even for the oldest stars (House et al. 2011).

We have test if these results are reliable by testing if STECKMAP is able to recover the age metallicity relation. We performed a series of experiments where a synthetic spectra of simulated star formation histories with different metallicity evolution were introduced as input in STECKMAP, previously degraded to the resolution of our data and adding noise to reach our Voronoi limit of 40. These tests are shown in Appendix A. As can be seen, although the scatter is larger at old ages, we can reproduce the input trend and recover the age-metallicity relation for synthetic data with similar characteristics than the data used in this study.

\subsection{Comparison of the gradients with the bar properties}

The changes in angular momentum predicted in numerical simulations are larger for stronger bars (e.g., Minchev \& Famaey 2010). Figure 10 shows the metallicity and age gradient $v s$. the strength of the bar. As can be seen, we do not find any correlation between the slope of the stellar population properties and the properties of the bar.

\section{Metallicity gradients for stellar populations of different ages}

Inside-out formation may lead to some specific evolution of the abundance gradients. How do the abundance gradients evolve within the disc? While most galactic chemical evolution models are able to reproduce the present-day radial distribution of several chemical elements derived from a sample objects representative of the present-day composition of the interstellar medium, they generally disagree on the predicted behaviour of its time evolution (compare, e.g., the models of Tosi 1988; Chiappini et al. 1997, 2001 - which predict a steeping of the gradients with 


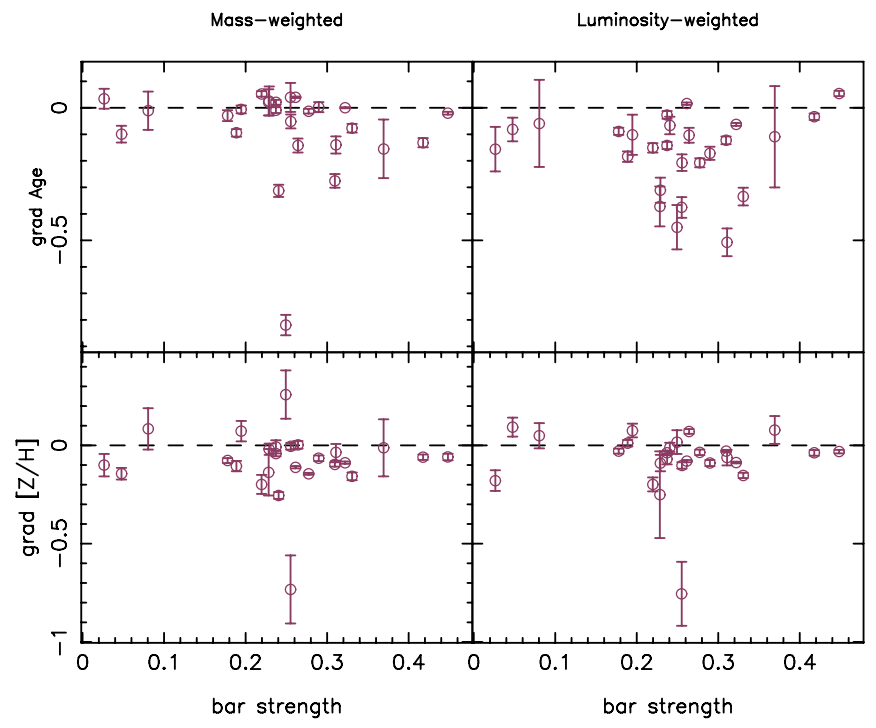

Fig. 10. Comparison of the metallicity gradients (measured in dex $/ r_{\text {eff }}$ ) of barred galaxies vs. the strength of the bar.

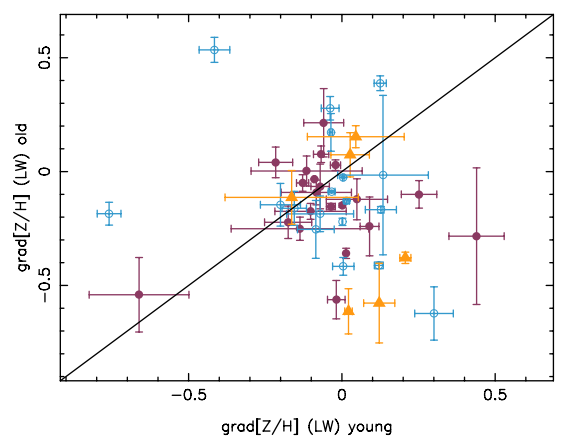

Fig. 11. Comparison of the metallicity gradient for the stellar population with an age older and younger than 6 Gyr. The colours and the symbols are the same as in Fig. 6.

time; with those of Mollá et al. 1997; Portinari \& Chiosi 1999 and Hou et al. 2000 - which predict a flattening with time). The main differences between the two "types" of models are the efficiency of the enrichment processes in the inner and outer regions of the disc and the degree of enrichment of the infall material. The same happens with fully cosmological hydrodynamical simulations. Pilkington et al. (2012) analysed the metallicity gradient and its evolution with time in a suite of 25 cosmological simulations of disc galaxies with similar properties to the Milky Way finding that, whilst the majority of the models predict radial gradients today which are consistent with those observed in late-type discs, they evolve in different fashion. They also found that the main differences came from the radial efficiency of the star formation rate that are controlled, mainly, by the different prescriptions of feedback (Gibson et al. 2013).

Up to now, these predictions could not be constrained due to the lack of observational results. Because the output of STECKMAP gives us the whole evolution of the metallicity with time, we are able to obtain the metallicity gradient for population of different ages (see Appendix A for tests supporting the reability of the results). With the same methodolgy that we used to obtain gradients for the whole population, we measure the metallicity gradient for stars with ages greater than $6 \mathrm{Gyr}$ and lower than 2 Gyr. Figure 11 compares the slopes of these two populations for the same galaxies whilst, in Fig. 12 we show the distribution of the slopes. As can be seen, the metallicity gradient for the young component is flatter (and, in many cases, positive) compared with that of the old component. However. the differences are not statistically significant. The mean values are $(0.002 \pm 0.208)$ and $(-0.005 \pm 0.262) \mathrm{dex} / r_{\mathrm{eff}}$ for the young and the old component respectively, where the quoted error represent the rms dispersion. Flatter abundance gradients in the younger population are also found in the Milky Way (Friel et al. 2002; Chen et al. 2003; Maciel et al. 2003; Daflon \& Cunha 2004), although recent results by Maciel \& Costa (2013) do not detect variations in the slope of the metallicity gradient measured in planetary nebulae of different ages. We do not find, either, any difference in the mean of the gradients for the old and young populations between galaxies with and without bars.

\section{Relation between the stellar populations of discs and bulges}

We have seen, in previous sections, that the stellar population gradients in the disc region of our sample do not correlate with other properties of the galaxies. This does not mean that the characteristic values of the stellar age and metallicities in the disc regions do not correlate with other properties. Figure 13 shows the values of the mean age and metallicities calculated at $1.5 r_{\mathrm{eff}}$ ( $\sim 2.5$ scale-lengths) as a function of the central velocity dispersion. The values of the central velocity dispersion have been calculated using the high spectral resolution version of the CALIFA data and will be presented in Falcón-Barroso et al. (in prep.). As can be seen, there is a relation for which galaxies with higher velocity dispersion have older and more metal rich populations in the disc. We have also plotted the central values of the galaxies for comparison. The relations between the age and metallicity and the velocity dispersion followed by the bulge and the disc are similar, with bulges older, on average, and more metal rich. Note that the dispersion in the luminosity-weighted age at a given central velocity dispersion is larger for the bulges than for the discs.

Figure 14 shows the relation between the stellar populations at $1.5 r_{\text {eff }}$ and that in the centre for our sample of galaxies. Both parameters are highly correlated. Correlations between the structural parameters and colours between the bulge and the disc have been found by previous authors (Peletier \& Balcells 1996; Courteau et al. 1996; de Jong 1996b; Carollo et al. 2007; Gadotti \& Dos Anjos 2001) and have been interpreted as evidences of internal secular evolution. However, the relation can also be driven by a third, global parameter that drives both the star formation history of bulges and discs. The stellar populations in the bulge of this sample will be the subject of a future paper and we wont discuss this issue further.

\section{Discussion}

In the present paper, we have analysed the spatially resolved stellar population properties in the disc of galaxies with and without bars to test observationally numerical simulations predicting that the non-linear coupling between bars and spiral waves can make the process of radial migration due to resonant scattering of resonances much more efficient and fast than when a single perturber (e.g., spiral arms alone) is considered (Friedli \& Benz 1995; Friedli 1998; Minchev \& Famaey 2010; Minchev et al. 2011a; Brunetti et al. 2011; Di Matteo et al. 2013).

In our analysis, we do not find any difference in the slope of the stellar-phase metallicity gradients between barred and unbarred galaxies. Does this mean that the non-linear coupling of 


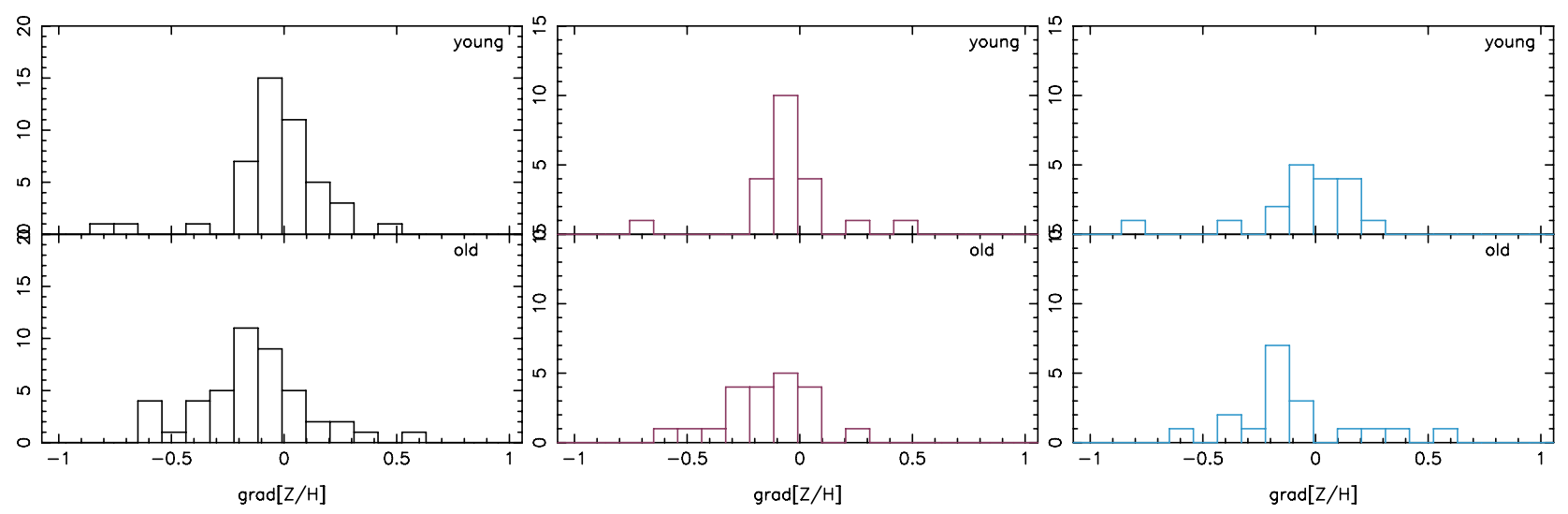

Fig. 12. Mean luminosity-weighted metallicity gradients for stellar populations with ages older and younger than 6 Gyr. Black histogram represent the whole sample whilst the blue and the red histograms show the results for unbarred and barred galaxies respectively.
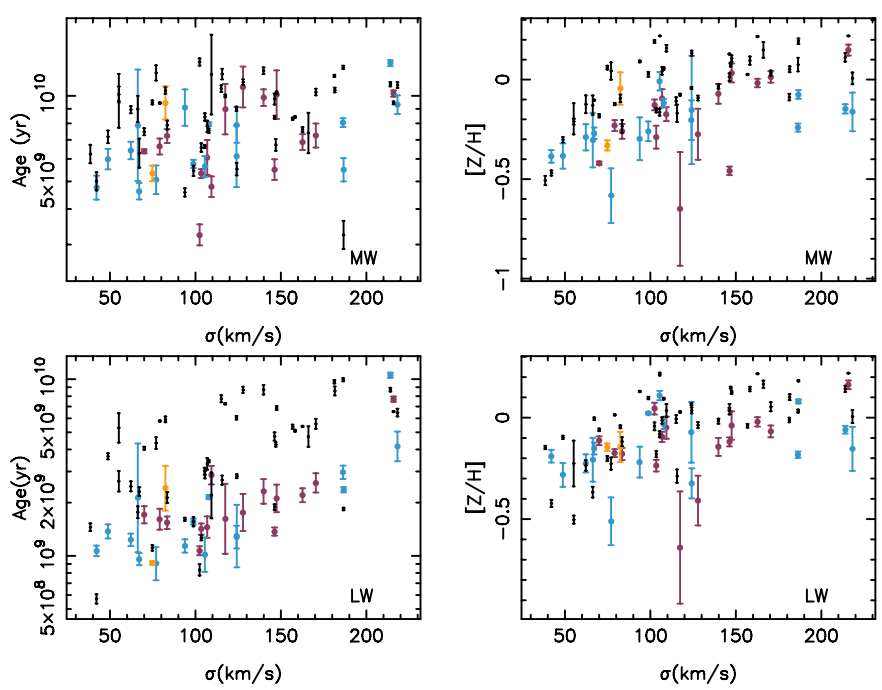

Fig. 13. Relation between the luminosity- and mass-weighted age and metallicity values calculated at $1.5 r_{\text {eff }}$ and the centr1al velocity dispersion. The meaning of the colours is the same as in previous figures. We have overplotted, with black symbols, the values calculated in the central spaxel.

bars and spirals is much weaker than what has been proposed so far and, therefore, that numerical simulations are failing at predicting the influence of bars on the evolution of the stellar disc? Not necessarily. Here we discuss some possibilities that can explain the lack of differences in our data.

(i) Radial migration due to the exchange of angular momentum at corotation is not efficient in hot discs (Brunetti et al. 2010). Brunetti et al. (2011) showed that not all barred galaxies experience strong diffusion and that the efficiency of the diffusion depends on the bar strength and thus, ultimately, on the stability of the disc. Hot discs do not respond to the perturbation created by the non-linear coupling of bar and spiral arms. If this is true we will expect to find the differences between barred and unbarred galaxies in those discs with lower vertical dispersion $\left(\sigma_{z}\right)$. However, we have compared the metallicity gradients for those galaxies with low $\sigma_{z}$ (using the methodology described in Gerssen 1997) without finding any difference.

(ii) The lack of differences between the gradients of barred and unbarred galaxies could be related with the longevity of bars. If bars are not long-lasting structures but recurrent
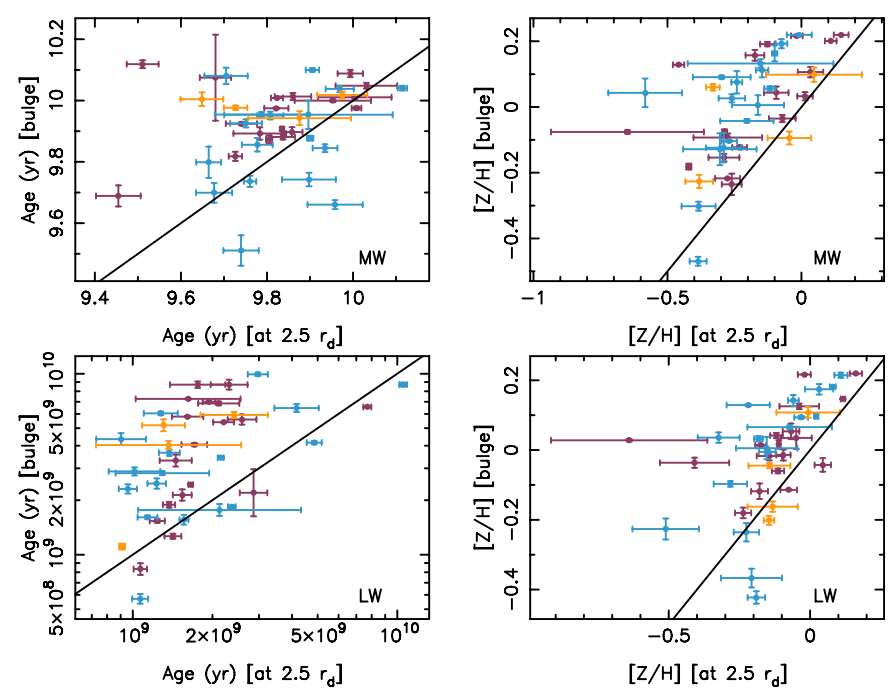

Fig. 14. Relation between the mean, luminosity- and mass-weighted, stellar population models measured at $1.5 r_{\mathrm{eff}}$ and in the centre of the galaxies of our sample. The meaning of the colours is the same as in previous figures.

patterns (Bournaud \& Combes 2002) then, the fact that we do not find differences between barred and unbarred galaxies would not necessarily imply that bars are not important for stellar migrations but, simply, that unbarred galaxies could have been barred in the recent past. However, most numerical simulations show that once they formed, bars are robust structures (Shen \& Sellwood 2004; Athanassoula et al. 2005, 2013; Debattista et al. 2006; Berentzen et al. 2007; Villa-Vargas et al. 2010; Kraljic et al. 2012). Kraljic et al. (2012) showed, in a series of cosmological simulated discs that the majority of bars formed at redshifts $z \sim 0.8-1$ survive to redshift $z=0$ without changing its strength, although cosmological gas infall is necessary to maintain some of them. They also showed that some bars formed earlier, when the gas fraction of the galaxies was higher, get destroyed and refurbish again but, again, these galaxies represent a minority of barred galaxies.

Furthermore, at least in massive disc galaxies, bars have old and metal rich stellar populations, older than the disc and similar in age and metallicity to that of the bulges (see Sánchez-Blázquez et al. 2011 and Pérez et al. 2009) which also support the idea that formed long time ago. The 
longevity of bars is also suggested in studies of bar fraction evolution with redshift (e.g., Sheth et al. 2008) that find a similar bar fraction at $z \sim 0.8$ than at $z=0.0$ for galaxies with $M_{*} \geq 10^{11} M_{\odot}$. Lower mass galaxies, however, have a bar fraction that was much lower at $z \sim 0.8$ than now, which can be explained with a delay in the bar formation (Athanassoula et al. 2013; Sheth et al. 2012). However, the debate regarding the survability of bars is still not closed and, therefore, the longevity of the bars remains a possibility to explain the lack of differences in the metallicity gradient.

(iii) In the theory of the corotation scattering mechanism described in Sellwood \& Binney (2002), the migration is only effective if the structure is transient. Bars then could not be effective at disc mixing once they were formed, due to their long-lived nature. However, recent $N$-body Tree-SPH simulations by Minchev et al. (2012a,b) show that bars are the most effective drivers of radial migration through the galactic evolution despite the fact that they are not transient, but only slowly evolving. This is also consistent with the findings by Brunetti et al. (2011).

(iv) Another possibility is that there is radial migration but the consequences are not visible in the metallicity gradient. This could happen, for example, if the metallicity gradient is not very steep (and it was also not very steep in the past). We have found, indeed, fairly flat present-day metallicity gradients, which could support this possibility. In our study of the evolution of the metallicity gradient, we have found steeper slopes for older populations but still, not very steep (except in some galaxies). Furthermore, we cannot discard a flattening of the gradients due to, precisely, stellar migrations. The only way to check this point is studying the gas-phase metallicity gradient at different redshifts. In the last years, several authors have obtained radial abundance gradients at high redshifts (Cresci et al. 2010; Jones et al. 2010; Queyrel et al. 2012; Yuan et al. 2011). Yuan et al. (2011) show that at least on one Grand Design disc at redshift $z$ 1.5, the metallicity gradient is significantly steeper $(-0.16 \mathrm{dex} / \mathrm{kpc})$ than the typical gradient encounter today. However, despite such advances, it is still very difficult to obtain high resolution data for galaxies at high redshift and, therefore, a flat metallicity gradient throughout the evolution of galactic discs remains a possibility to explain the lack of differences in the metallicity gradients of barred and unbarred galaxies.

The results can also indicate that the mechanism proposed by Minchev \& Famaey (2010) is not as important as producing radial migrations as predicted. This does not mean that radial migration is not important. Other mechanism not related with the presence of bars can be at play. Sellwood \& Binney (2002) suggested a mechanism based on resonant scattering of stars under the effect of transient spiral waves (see Sect. 1). Haywood (2008) estimated upper values for the migration rate from 1.5 to $3.7 \mathrm{kpc} / \mathrm{Gyr}$, which agree with the values in Lépine et al. (2003) for the radial wandering due to the scattering mechanism assumed by Sellwood \& Binney (2002). Observationally, claimed evidences of stellar migration, as upturns in the age distribution of stars have been reported in galaxies without bars (e.g., de Jong et al. 2007; Yoachim et al. 2010, 2012, Radburn-Smith 2012). However, it was shown in Sánchez-Blázquez et al. (2009) that an upturn in the age distribution beyond the break is not an unequivocal sign of radial migrations and that this can be produced by other reasons, for example, a warp in the gaseous discs that decrease the star formation density in the external parts. Furthermore, there are many galaxies with breaks where the upturn in the stellar population has not been observed (e.g., Gorgarten et al. 2010; Yoachim et al. 2012). The stellar migration models should explain why this is the case. Another "evidence" of the occurrence of radial migration is the slope and scatter of the age-metallicity relation in the solar neighbourhood (i.e., Roškar et al. 2008b). However, this can be consequence of the errors in the determination of ages and abundances in individual stars (Anguiano et al., in prep.). However, considerably work remains to be done and all of these conclusions remain speculative.

To finish we want to stress here that our results do not contradict previous observational results in the field. Studies of stellar populations in disc galaxies comparing galaxies with and without bars have only been done using colours (Gadotti \& Dos Anjos 2001). This study found that there was an excess of barred galaxies among the objects with null or positive (bluish inward) colour gradients. However, the gradients were calculated in the whole galaxy, not just the disc (as in our study) and, therefore, are strongly influenced by the colour differences between the bulge and the disc.

On the other hand, previous studies comparing the gas-phase metallicity gradient of galaxies with and without bars had found flatter gradients in barred galaxies compared with unbarred ones (e.g., Martin \& Roy 1994; Zaritsky et al. 1994). However, gas and stars suffer from very different evolutionary processes; the gas is mainly dominated by the gravitational torque of the nonaxisymmetric mass component, whilst the stars are mainly affected by different orbital mixing. Furthermore, the above cited studies suffer from the poor number statistics (only 5 barred galaxies were analysed in Zaritski et al.). Using a much larger sample of 306 galaxies from the CALIFA survey, Sánchez et al. (2014) found no difference between the gas-phase metallicity gradient of barred and unbarred galaxies.

\section{Conclusions}

We have presented a spatially resolved analysis of the star formation histories and metallicity evolution for a sample of disc galaxies using data from the CALIFA survey. The main motivation of this study is to compare the stellar population gradients in galaxies with and without bars, to analyse the influence of these axisymmetric structures in producing radial migrations. However, we also present generalities related with the relation of the stellar population gradients and other properties of the galaxies and the evolution of the metallicity gradients with time.

The main conclusions of the present study are:

- The mass-weighted age in disc galaxies reflect the fact that nearby galaxies in the mass range studied by us are dominated, at all radii, by old stars.

- The luminosity-weighted age profile show a shallow, but negative slope for the majority of our galaxies. The mean values are $-0.116 \pm 0.200$ and $-0.014 \pm 0.135 \mathrm{dex} / r_{\text {eff }}$ for the light- and the mass-weighted values. This implies that there is a higher proportion of young vs. old stars in the external parts of the disc with respect to the inner parts.

- The mean luminosity-weighted metallicity gradient is also shallow when normalised to the effective radius of the disc. In most cases, the luminosity-weighted metallicity gradient is steeper than the mass-weighted one. The mean values are $-0.051 \pm 0.126 \mathrm{dex} / r_{\mathrm{eff}}$ and $-0.089 \pm 0.151 \mathrm{dex} / r_{\mathrm{eff}}$ for the mass and the light-weighted gradientes respectively, where the errors represent the rms dispersion. 
- The analysis of the metallicity gradients at different stellar ages reflects steeper gradients for older stars.

- The stellar-metallicity gradient (normalised to the effective radius of the disc) is not correlated with other global galactic properties, as the morphological type the mass or the luminosity.

- We compare the stellar-metallicity gradients of galaxies with and without bars and we did not find any significant difference. This is contrary to the predictions of some numerical simulations indicating a flattening of the metallicity gradient due to the bar presence.

Acknowledgements. We wish to thank Brad Gibson and Pierre Ocvirk for their constant advice. P.S.-B. acknowledges support from the Ramón y Cajal program, grant ATA2010-21322-C03-02 from the Spanish Ministry of Economy and Competitiveness (MINECO). F.F.R.O. acknowledges the Mexican National Council for Science and Technology (CONACYT) for financial support under the programme Estancias Posdoctorales y Sabáticas al Extranjero para la Consolidación de Grupos de Investigación, 2010-2012. We acknowledge financial support from the research projects under grants AYA2010-21887-C0403, AYA2010-21322-C03-03 and AYA2010-15081 by the Spanish Ministerio de Ciencia e Innovación. J.F.-B. acknowledges support from the Ramon y Cajal Program, grants AYA2010-21322-C03-02 from the Spanish Ministry of Economy and Competitiveness (MINECO). R.A.M. is funded by the Spanish program of International Campues of Excellence Moncloa (CEI). C.J.W. acknowledges support through the Marie Curie Career Integration Grant 303912. I.M. acknowledges financial support from the Junta de Andalucía through projects PO8-TIC-03531 and TIC114, and the Spanish Ministry of Economy and Competitiveness (MINECO) through the project AYA2010-15169. We acknowledge the usage of the HyperLeda database (http://leda . univ-lyon 1. $\mathrm{fr})$. Based on observations collected at the Centro Astronímico HispanoAlean (CAHA) at Calar Alto, operated jointly by the Max-Planck Institut ur Astronomie and the Instituto de Astrofísica de Andaluca (CSIC). FCT/MCTES (Portugal) and POPH/FSE (EC). He acknowledges support by the Fundação para a Ciência e a Tecnologia (FCT) under project FCOMP-01-0124-FEDER-029170 (Reference FCT PTDC/FIS-AST/3214/2012), funded by FCT-MEC (PIDDAC) and FEDER (COMPETE). We also thanks the referee por his/her suggestions that have improved the final version of this paper.

\section{References}

Abazajian, K. N., Adelman-McCarthy, J. K., Agüeros, M. A., et al. 2009, ApJS, 182,543

Abraham, R. G., \& Merrifield, M. R. 2000, AJ, 120, 2835

Aguerri, J. A. L., Méndez-Abreu, J., \& Corsini, E. M. 2009, A\&A, 495, 491

Alonso, A., Arribas, S., \& Martinez-Roger, C. 1996, A\&A, 313, 873

Athanassoula, E., Lambert, J. C., \& Dehnen, W. 2005, MNRAS, 363, 496

Athanassoula, E., Machado, R. E. G., \& Rodionov, S. A. 2013, MNRAS, 429, 1949

Barazza, F. D., Jogee, S., \& Marinova, I. 2008, ApJ, 675, 1194

Barker, M. K., Sarajedini, A., Geisler, D., Harding, P., \& Schommer, R. 2007, AJ, 133, 1138

Beauchamp, D., \& Hardy, E. 1997, AJ, 113, 1666

Bell, E. F., \& de Jong, R. S. 2000, MNRAS, 312, 497

Berentzen, I., Shlosman, I., Martinez-Valpuesta, I., \& Heller, C. H. 2007, ApJ, 666, 189

Bertelli, G., Bressan, A., Chiosi, C., Fagotto, F., \& Nasi, E. 1994, A\&AS, 106, 275

Brunetti, M., Chiappini, C., \& Pfenniger, D. 2011, A\&A, 534, A75

Bruzual, G., \& Charlot, S. 2003, MNRAS, 344, 1000

Buta, R., \& Block, D. L. 2001, ApJ, 550, 243

Cappellari, M., \& Copin, Y. 2003, MNRAS, 342, 345

Cappellari, M., \& Emsellem, E. 2004, PASP, 116, 138

Carollo, C. M., Scarlata, C., Stiavelli, M., Wyse, R. F. G., \& Mayer, L. 2007, ApJ, 658, 960

Carraro, G., Geisler, D., Villanova, S., Frinchaboy, P. M., \& Majewski, S. R. 2007, A\&A, 476, 217

Cenarro, A. J., Peletier, R. F., Sánchez-Blázquez, P., et al. 2007, MNRAS, 374, 664

Chen, L., Hou, J. L., \& Wang, J. J. 2003, AJ, 125, 1397

Chiappini, C., Matteucci, F., \& Gratton, R. 1997, ApJ, 477, 765

Chiappini, C., Matteucci, F., \& Romano, D. 2001, ApJ, 554, 1044
Cid Fernandes, R., Mateus, A., Sodré, L., Stasińska, G., \& Gomes, J. M. 2005, MNRAS, 358, 363

Cid Fernandes, R., Pérez, E., García Benito, R., et al. 2013, A\&A, 557, A86

Cid Fernandes, R., González Delgado, R. M., García Benito, R., et al. 2014 A\&A, 561, A130

Cioni, M.-R. L. 2009, A\&A, 506, 1137

Comparetta, J., \& Quillen, A. C. 2012 [arXiv: 1207. 5753]

Cresci, G., Mannucci, F., Maiolino, R., et al. 2010, Nature, 467, 811

Daflon, S., \& Cunha, K. 2004, ApJ, 617, 1115

Davidge, T. J. 2006, PASP, 118, 1626

Davidge, T. J. 2007, ApJ, 664, 820

de Jong, R. S. 1996, A\&A, 313, 377

de Jong, R. S., Seth, A. C., Radburn-Smith, D. J., et al. 2007, ApJ, 667, L49

Debattista, V. P., Mayer, L., Carollo, C. M., et al. 2006, ApJ, 645, 209

Diaz, A. I. 1989, in Evolutionary Phenomena in Galaxies, eds. J. E. Beckman, \& B. E. J. Pagel (Cambridge, New York: Cambridge University press), 377

Di Matteo, P., Haywood, M., Combes, F., Semelin, B., \& Snaith, O. N. 2013, A\&A, 553, A102

Eskridge, P. B., Frogel, J. A., Pogge, R. W., et al. 2000, AJ, 119, 536

Falcón-Barroso, J., Sánchez-Blázquez, P., Vazdekis, A., et al. 2011, A\&A, 532, A95

Freeman, K., \& Bland-Hawthorn, J. 2002, ARA\&A, 40, 487

Friedli, D. 1998, in Abundance Profiles: Diagnostic Tools for Galaxy History, eds. D. Friedli, M. Edmunds, C. Robert, \& L. Drissen, ASP Conf. Ser., 147, 287

Friedli, D., Benz, W., \& Kennicutt, R. 1994, ApJ, 430, L105

Friel, E. D., Janes, K. A., Tavarez, M., et al. 2002, AJ, 124, 2693

Gadotti, D. A., \& dos Anjos, S. 2001, AJ, 122, 1298

Ganda, K., Peletier, R. F., Balcells, M., \& Falcón-Barroso, J. 2009, MNRAS, 395,1669

Gerssen, J., Kuijken, K., \& Merrifield, M. R. 1997, MNRAS, 288, 618

Gibson, B. K., Pilkington, K., Brook, C. B., Stinson, G. S., \& Bailin, J. 2013, A\&A, 554, A47

Girardi, L., Bressan, A., Bertelli, G., \& Chiosi, C. 2000, A\&AS, 141, 371

Goetz, M., \& Koeppen, J. 1992, A\&A, 262, 455

Gogarten, S. M., Dalcanton, J. J., Williams, B. F., et al. 2010, ApJ, 712, 858

González Delgado, R. M., Pérez, E., Cid Fernandes, R., et al. 2014, A\&A, 562, A47

Grand, R. J. J., Kawata, D., \& Cropper, M. 2012a, MNRAS, 426, 167

Grand, R. J. J., Kawata, D., \& Cropper, M. 2012b, MNRAS, 421, 1529

Heavens, A. F., Jimenez, R., \& Lahav, O. 2000, MNRAS, 317, 965

Hou, J. L., Prantzos, N., \& Boissier, S. 2000, A\&A, 362, 921

House, E. L., Brook, C. B., Gibson, B. K., et al. 2011, MNRAS, 415, 2652

Hughes, S. M. G., Stetson, P. B., Turner, A., et al. 1994, ApJ, 428, 143

Husemann, B., Jahnke, K., Sánchez, S. F., et al. 2013, A\&A, 549, A87

Jansen, R. A., Fabricant, D., Franx, M., \& Caldwell, N. 2000, ApJS, 126, 331

Jones, T., Ellis, R., Jullo, E., \& Richard, J. 2010, ApJ, 725, L176

Koleva, M., Prugniel, P., Ocvirk, P., Le Borgne, D., \& Soubiran, C. 2008, MNRAS, 385, 1998

Koleva, M., de Rijcke, S., Prugniel, P., Zeilinger, W. W., \& Michielsen, D. 2009, MNRAS, 396, 2133

Kraljic, K., Bournaud, F., \& Martig, M. 2012, ApJ, 757, 60

Krauss, L. M., \& Chaboyer, B. 2003, Science, 299, 65

Kroupa, P. 2001, MNRAS, 322, 231

Kubryk, M., Prantzos, N., \& Athanassoula, E. 2013, MNRAS, 436, 1479

Kudritzki, R.-P., Urbaneja, M. A., Bresolin, F., et al. 2008, ApJ, 681, 269

Laurikainen, E., Salo, H., Buta, R., \& Knapen, J. H. 2007, MNRAS, 381, 401

Lépine, J. R. D., Acharova, I. A., \& Mishurov, Y. N. 2003, ApJ, 589, 210

Loebman, S. R., Roškar, R., Debattista, V. P., et al. 2011, ApJ, 737, 8

MacArthur, L. A. 2005, ApJ, 623, 795

MacArthur, L. A., Courteau, S., Bell, E., \& Holtzman, J. A. 2004, ApJS, 152, 175

MacArthur, L. A., González, J. J., \& Courteau, S. 2009, MNRAS, 395, 28

Maciel, W. J., \& Costa, R. D. D. 2013, Rev. Mex. Astron. Astrofis., 49, 333

Maciel, W. J., Costa, R. D. D., \& Uchida, M. M. M. 2003, A\&A, 397, 667

Maraston, C., \& Strömbäck, G. 2011, MNRAS, 418, 2785

Matteucci, F., \& Francois, P. 1989, MNRAS, 239, 885

McCall, M. L., Rybski, P. M., \& Shields, G. A. 1985, ApJS, 57, 1

Méndez-Abreu, J., Sánchez-Janssen, R., \& Aguerri, J. A. L. 2010, ApJ, 711, L61

Méndez-Abreu, J., Sánchez-Janssen, R., Aguerri, J. A. L., Corsini, E. M., \& Zarattini, S. 2012, ApJ, 761, L6

Minchev, I., \& Famaey, B. 2010, ApJ, 722, 112

Minchev, I., Famaey, B., Combes, F., et al. 2011, A\&A, 527, A147

Minchev, I., Famaey, B., Quillen, A. C., et al. 2012a, A\&A, 548, A127

Minchev, I., Famaey, B., Quillen, A. C., et al. 2012b, A\&A, 548, A126

Minchev, I., Chiappini, C., \& Martig, M. 2014a [arXiv: 1401.5796]

Minchev, I., Chiappini, C., \& Martig, M. 2014b, IAU Symp., 298, 130 
Molla, M., Ferrini, F., \& Diaz, A. I. 1997, ApJ, 475, 519

Mollá, M., Hardy, E., \& Beauchamp, D. 1999, ApJ, 513, 695

Monteverde, M. I., Herrero, A., Lennon, D. J., \& Kudritzki, R.-P. 1997, ApJ, 474, L107

Muñoz-Mateos, J. C., Gil de Paz, A., Boissier, S., et al. 2007, ApJ, 658, 1006

Muñoz-Mateos, J. C., Gil de Paz, A., Zamorano, J., et al. 2009, ApJ, 703, 1569

Nair, P. B., \& Abraham, R. G. 2010, ApJ, 714, L260

Ocvirk, P. 2010, ApJ, 709, 88

Ocvirk, P., Pichon, C., Lançon, A., \& Thiébaut, E. 2006a, MNRAS, 365, 74

Ocvirk, P., Pichon, C., Lançon, A., \& Thiébaut, E. 2006b, MNRAS, 365, 46

Oey, M. S., \& Kennicutt, Jr., R. C. 1993, ApJ, 411, 137

Panter, B., Heavens, A. F., \& Jimenez, R. 2003, MNRAS, 343, 1145

Peletier, R. F., \& Balcells, M. 1996, AJ, 111, 2238

Percival, S. M., \& Salaris, M. 2009, ApJ, 703, 1123

Pérez, E., Cid Fernandes, R., González Delgado, R. M., et al. 2013, ApJ, 764, L1

Pérez, I., Sánchez-Blázquez, P., \& Zurita, A. 2009, A\&A, 495, 775

Pilkington, K., Few, C. G., Gibson, B. K., et al. 2012, A\&A, 540, A56

Portinari, L., \& Chiosi, C. 1999, A\&A, 350, 827

Prochaska, J. X., O’Meara, J. M., \& Worseck, G. 2010, ApJ, 718, 392

Queyrel, J., Contini, T., Kissler-Patig, M., et al. 2012, A\&A, 539, A93

Radburn-Smith, D. J., Roškar, R., Debattista, V. P., et al. 2012, ApJ, 753, 138

Rosales-Ortega, F. F., Kennicutt, R. C., Sánchez, S. F., et al. 2010, MNRAS, 405, 735

Rosales-Ortega, F. F., Sánchez, S. F., Iglesias-Páramo, J., et al. 2012, ApJ, 756 , L31

Roškar, R., Debattista, V. P., Quinn, T. R., Stinson, G. S., \& Wadsley, J. 2008a, ApJ, 684, L79

Roškar, R., Debattista, V. P., Stinson, G. S., et al. 2008b, ApJ, 675, L65

Ryder, S. D., Fenner, Y., \& Gibson, B. K. 2005, MNRAS, 358, 1337

Salpeter, E. E. 1955, ApJ, 121, 161

Sánchez, S. F., Kennicutt, R. C., Gil de Paz, A., et al. 2012a, A\&A, 538, A8

Sánchez, S. F., Rosales-Ortega, F. F., Marino, R. A., et al. 2012b, A\&A, 546, A2

Sánchez, S. F., Rosales-Ortega, F. F., Iglesias-Páramo, J., et al. 2014, A\&A, 563, A49

Sánchez-Blázquez, P., Peletier, R. F., Jiménez-Vicente, J., et al. 2006, MNRAS, 371,703

Sánchez-Blázquez, P., Courty, S., Gibson, B. K., \& Brook, C. B. 2009, MNRAS, 398,591
Sánchez-Blázquez, P., Ocvirk, P., Gibson, B. K., Pérez, I., \& Peletier, R. F. 2011 MNRAS, 415, 709

Sánchez-Blázquez, P., Rosales-Ortega, F., Diaz, A., \& Sánchez, S. F. 2014, MNRAS, 437, 1534

Sarzi, M., Falcón-Barroso, J., Davies, R. L., et al. 2006, MNRAS, 366, 1151

Schiavon, R. P., Faber, S. M., Rose, J. A., \& Castilho, B. V. 2002, ApJ, 580, 873

Schönrich, R., \& Binney, J. 2009, MNRAS, 399, 1145

Sellwood, J. A., \& Binney, J. J. 2002, MNRAS, 336, 785

Shen, J., \& Sellwood, J. A. 2004, ApJ, 604, 614

Sheth, K., Elmegreen, D. M., Elmegreen, B. G., et al. 2008, ApJ, 675, 1141

Sheth, K., Melbourne, J., Elmegreen, D. M., et al. 2012, ApJ, 758, 136

Shevchenko, I. I. 2011, ApJ, 733, 39

Sygnet, J. F., Tagger, M., Athanassoula, E., \& Pellat, R. 1988, MNRAS, 232, 733

Tagger, M., Sygnet, J. F., Athanassoula, E., \& Pellat, R. 1987, ApJ, 318, L43

Taylor, V. A., Jansen, R. A., Windhorst, R. A., Odewahn, S. C., \& Hibbard, J. E. 2005, ApJ, 630, 784

Tojeiro, R., Heavens, A. F., Jimenez, R., \& Panter, B. 2007, MNRAS, 381, 1252 Tosi, M. 1988, A\&A, 197, 33

Urbaneja, M. A., Herrero, A., Bresolin, F., et al. 2005, ApJ, 622, 862

Vazdekis, A., Salaris, M., Arimoto, N., \& Rose, J. A. 2001, ApJ, 549, 274

Vazdekis, A., Sánchez-Blázquez, P., Falcón-Barroso, J., et al. 2010, MNRAS, 404, 1639

Vila-Costas, M. B., \& Edmunds, M. G. 1992, MNRAS, 259, 121

Villa-Vargas, J., Shlosman, I., \& Heller, C. 2010, ApJ, 719, 1470

Vlajić, M., Bland-Hawthorn, J., \& Freeman, K. C. 2009, ApJ, 697, 361

Walcher, J., Groves, B., Budavári, T., \& Dale, D. 2011, Ap\&SS, 331, 1

Wielen, R. 1977, A\&A, 60, 263

Williams, B. F., Dalcanton, J. J., Dolphin, A. E., Holtzman, J., \& Sarajedini, A. 2009a, ApJ, 695, L15

Williams, B. F., Dalcanton, J. J., Seth, A. C., et al. 2009b, AJ, 137, 419

Worthey, G., España, A., MacArthur, L. A., \& Courteau, S. 2005, ApJ, 631, 820

Yoachim, P., Roškar, R., \& Debattista, V. P. 2010, ApJ, 716, L4

Yoachim, P., Roškar, R., \& Debattista, V. P. 2012, ApJ, 752, 97

Yong, D., Carney, B. W., Teixera de Almeida, M. L., \& Pohl, B. L. 2006, AJ, 131,2256

York, D. G., Adelman, J., Anderson, Jr., J. E., et al. 2000, AJ, 120, 1579

Yuan, T.-T., Kewley, L. J., Swinbank, A. M., Richard, J., \& Livermore, R. C. 2011, ApJ, 732, L14

Pages 15 to 85 are available in the electronic edition of the journal at http://www . aanda. org 
Table 5. Main characteristics of the sample.

\begin{tabular}{|c|c|c|c|c|c|c|c|c|}
\hline & CALIFA & Morph & $r_{\mathrm{bar}}\left({ }^{\prime \prime}\right)$ & $f_{\mathrm{bar}}$ & $\log M_{*}$ & $r_{\mathrm{eff}}\left({ }^{\prime \prime}\right)$ & t-type & Inc \\
\hline IC 1256 & 856 & $\mathrm{Sb}(\mathrm{AB})$ & 10.6 & 0.20 & 9.873 & 17.3 & 3.3 & 56.2 \\
\hline IC 1683 & 043 & $\mathrm{Sb}(\mathrm{AB})$ & 11.2 & 0.03 & 10.517 & 12.0 & 2.7 & 56.5 \\
\hline NGC 0001 & 008 & $\operatorname{Sbc}(A)$ & - & - & 10.656 & 17.7 & 3.1 & 37.9 \\
\hline NGC 0036 & 010 & $\mathrm{Sb}(\mathrm{B})$ & 24.2 & 0.30 & 10.914 & 31.7 & 3.0 & 51.5 \\
\hline NGC 0160 & 020 & $\mathrm{Sa}(\mathrm{A})$ & - & - & 10.919 & 37.6 & -0.4 & 57.9 \\
\hline NGC 0214 & 028 & $\mathrm{Sbc}(\mathrm{AB})$ & 15.2 & 0.08 & 10.872 & 17.3 & 5.0 & 49.9 \\
\hline NGC 0234 & 031 & $\mathrm{Sc}(\mathrm{AB})$ & - & - & 10.597 & 18.4 & 5.3 & 32.3 \\
\hline NGC 0257 & 033 & $\mathrm{Sc}(\mathrm{A})$ & - & - & 10.795 & 19.4 & 5.8 & 56.2 \\
\hline NGC 0776 & 073 & $\mathrm{Sb}(\mathrm{B})$ & 18.2 & 0.26 & 10.720 & 18.0 & 2.5 & 48.8 \\
\hline NGC 0496 & 045 & $\operatorname{Scd}(A)$ & & & & & & \\
\hline NGC 1167 & 119 & SO(A) & - & - & 11.129 & 29.8 & -2.4 & 42.3 \\
\hline NGC 1645 & 134 & $\mathrm{~S} 0 \mathrm{a}(\mathrm{B})$ & 16.6 & 0.29 & 10.780 & 24.9 & -0.9 & 57.5 \\
\hline NGC 2253 & 147 & $\operatorname{Sbc}(\mathrm{B})$ & 14.5 & - & 10.547 & 8.4 & 5.8 & 30.4 \\
\hline NGC 2347 & 149 & $\mathrm{Sbc}(\mathrm{AB})$ & 14.6 & - & 10.759 & 18.5 & 3.1 & 52.4 \\
\hline NGC 2906 & 275 & $\operatorname{Sbc}(A)$ & - & - & 10.288 & 16.1 & 5.9 & 60.9 \\
\hline NGC 2916 & 277 & $\operatorname{Sbc}(A)$ & - & - & 10.421 & 25.3 & 3.1 & 56.7 \\
\hline NGC 3106 & 311 & $\mathrm{Sab}(\mathrm{A})$ & - & - & 11.001 & 22.7 & -1.9 & 24.4 \\
\hline NGC 3300 & 339 & $\mathrm{SOa}(\mathrm{B})$ & 17.0 & 0.26 & 10.526 & 11.5 & -1.8 & 60.0 \\
\hline NGC 3614 & 388 & $\operatorname{Sbc}(A B)$ & 32.0 & - & 9.937 & 41.1 & 5.2 & 45.1 \\
\hline NGC 3687 & 414 & $\mathrm{Sb}(\mathrm{B})$ & 15.2 & 0.19 & 10.040 & 20.1 & 3.8 & 24.3 \\
\hline NGC 4047 & 489 & $\operatorname{Sbc}(A)$ & - & - & 10.539 & 16.5 & 3.2 & 39.5 \\
\hline NGC 4185 & 515 & $\mathrm{Sbc}(\mathrm{AB})$ & 21.0 & 0.03 & 10.577 & 27.9 & 3.7 & 52.1 \\
\hline NGC 4210 & 518 & $\mathrm{Sb}(\mathrm{B})$ & 20.8 & 0.33 & 10.193 & 17.0 & 3.0 & 45.2 \\
\hline NGC 4470 & 548 & $\mathrm{Sc}(\mathrm{A})$ & - & - & 9.853 & 12.6 & 1.4 & 52.6 \\
\hline NGC 5000 & 608 & $\operatorname{Sbc}(B)$ & 27.7 & 0.37 & 10.545 & 18.2 & 3.8 & 55.0 \\
\hline NGC 5016 & 611 & $\operatorname{Sbc}(A)$ & - & - & 10.093 & 19.0 & 4.4 & 44.4 \\
\hline NGC 5205 & 630 & $\mathrm{Sbc}(\mathrm{B})$ & 21.4 & 0.28 & 9.728 & 21.1 & 3.5 & 50.3 \\
\hline NGC 5218 & 634 & $\operatorname{Sab}(B)$ & 18.5 & 0.31 & 10.469 & 17.6 & 3.1 & 58.9 \\
\hline NGC 5378 & 676 & $\mathrm{Sb}(\mathrm{B})$ & 34.2 & 0.25 & 10.335 & 24.6 & 1.0 & 55.5 \\
\hline NGC 5394 & - & $\operatorname{Sbc}(\mathrm{B})$ & 25.3 & - & 9.873 & 19.0 & 3.1 & 43.6 \\
\hline NGC 5406 & 684 & $\mathrm{Sb}(\mathrm{B})$ & 22.9 & 0.29 & 11.005 & 20.4 & 3.9 & 29.0 \\
\hline NGC 5614 & 740 & $\mathrm{Sa}(\mathrm{A})$ & - & - & 10.976 & 27.5 & 1.7 & 19.2 \\
\hline NGC 5633 & 748 & $\operatorname{Sbc}(\mathrm{A})$ & - & - & 10.097 & 12.6 & 3.2 & 52.2 \\
\hline NGC 5720 & 764 & $\operatorname{Sbc}(\mathrm{B})$ & 11.2 & 0.23 & 10.847 & 19.9 & 3.0 & 51.7 \\
\hline NGC 5732 & 768 & $\operatorname{Sbc}(A)$ & - & - & 9.792 & 16.2 & 4.0 & 56.5 \\
\hline NGC 5784 & 778 & $\mathrm{~S} 0(\mathrm{~A})$ & - & - & 10.985 & 22.3 & -2.0 & 42.1 \\
\hline NGC 6004 & 813 & $\operatorname{Sbc}(B)$ & 20.4 & 0.32 & 10.467 & 21.7 & 4.9 & 19.8 \\
\hline NGC 6063 & 823 & $\operatorname{Sbc}(\mathrm{A})$ & - & - & 9.908 & 20.7 & 5.9 & 54.1 \\
\hline NGC 6154 & 833 & $\operatorname{Sab}(B)$ & 31.8 & 0.28 & 10.734 & 20.0 & 1.0 & 54.0 \\
\hline NGC 6155 & 836 & $\mathrm{Sc}(\mathrm{A})$ & - & - & 9.958 & 13.8 & 5.2 & 48.2 \\
\hline NGC 6301 & 849 & $\operatorname{Sbc}(\mathrm{A})$ & - & - & 10.929 & 26.4 & 5.9 & 54.3 \\
\hline NGC 6497 & 863 & $\operatorname{Sab}(B)$ & 15.6 & 0.25 & 10.316 & 17.7 & 3.1 & 51.9 \\
\hline NGC 6941 & 869 & $\mathrm{Sb}(\mathrm{B})$ & 18.6 & 0.23 & 10.862 & 23.0 & 3.2 & 45.2 \\
\hline NGC 7025 & 874 & SOa(A) & - & - & 11.063 & 23.0 & 1.0 & 47.5 \\
\hline NGC 7321 & 887 & $\mathrm{Sbc}(\mathrm{B})$ & 14.1 & 0.22 & 10.984 & 23.0 & 3.1 & 48.7 \\
\hline NGC 7489 & 898 & $\operatorname{Sbc}(A)$ & - & - & 10.483 & 14.6 & 6.4 & 58.2 \\
\hline NGC 7549 & 901 & $\operatorname{Sbc}(B)$ & 31.2 & - & 10.539 & 11.9 & 5.9 & 42.1 \\
\hline NGC 7563 & 902 & $\mathrm{Sa}(\mathrm{B})$ & 25.9 & 0.28 & 10.753 & 10.0 & 1.0 & 50.5 \\
\hline NGC 7591 & 904 & $\operatorname{Sbc}(\mathrm{B})$ & 13.6 & 0.18 & 10.768 & 19.9 & 3.6 & 56.5 \\
\hline NGC 7653 & 915 & $\mathrm{Sb}(\mathrm{A})$ & - & - & 10.486 & 17.6 & 3.1 & 29.2 \\
\hline NGC 7671 & 916 & SO(A) & - & - & 10.786 & 14.0 & -2.0 & 60.0 \\
\hline NGC 7782 & 931 & $\mathrm{Sb}(\mathrm{A})$ & - & - & 11.096 & 26.0 & 3.0 & 59.7 \\
\hline UGC 00005 & 002 & $\operatorname{Sbc}(A)$ & - & - & 10.883 & 16.1 & 3.9 & 59.6 \\
\hline UGC 00036 & 007 & $\mathrm{Sab}(\mathrm{AB})$ & - & - & 10.781 & 16.2 & 1.0 & 57.9 \\
\hline UGC 03253 & 146 & $\mathrm{Sb}(\mathrm{B})$ & 17.4 & 0.25 & 10.397 & 18.2 & 3.0 & 53.9 \\
\hline UGC 07012 & 486 & $\operatorname{Scd}(\mathrm{AB})$ & 4.6 & - & 9.010 & 18.4 & 5.7 & 59.2 \\
\hline UGC 08234 & 607 & S0(A) & - & - & 11.061 & 9.0 & -0.1 & 56.6 \\
\hline UGC 10205 & 822 & SOa(A) & - & - & 10.947 & 17.9 & 1.0 & 59.8 \\
\hline UGC 11649 & 872 & $\mathrm{Sab}(\mathrm{B})$ & 23.3 & 0.25 & 10.467 & 18.2 & 1.0 & 30.3 \\
\hline UGC 11680 & 873 & $\mathrm{Sb}(\mathrm{B})$ & 12.9 & - & 10.892 & 33.6 & 3.0 & 41.5 \\
\hline UGC 12224 & 891 & $\mathrm{Sc}(\mathrm{A})$ & - & - & 9.751 & 31.5 & 5.0 & 34.3 \\
\hline UGC 12816 & 930 & $\mathrm{Sc}(\mathrm{A})$ & - & - & 9.649 & 20.2 & 5.8 & 53.0 \\
\hline
\end{tabular}

Notes. (1) Galaxy name; (2) CALIFA identifier; (3) visual morphological classification; (4) bar radius obtained as the mean value between three methods: (i) the maximum and the (ii) minimum of the ellipticity and the (iii) radius at which the position angle change by more than 5 degrees; (5) strength of the bar derived as explained in the text; (6) stellar masses (in logarithmic scale) derived by a SED fitting using Kcorrect; (7) effective radius of the disc in arcsec; (8) t-type classification using the modified RC3 classifiers; (9) inclination (in degrees), purely based on the ellipticity. 
Table 6. Luminosity- and mass-weighted $\log ($ age $)$ and metallicity gradient calculated as the slope of a linear fit of the form $a+b \log \left(r / r_{\text {eff }}\right.$ for our sample of galaxies.

\begin{tabular}{|c|c|c|c|c|}
\hline & \multicolumn{2}{|c|}{$\overline{\overline{[Z / H]}}$} & \multicolumn{2}{|c|}{$\overline{\text { loge Age }}$} \\
\hline & $\begin{array}{r}\text { Mass-weighted } \\
\text { slope }\end{array}$ & $\begin{array}{r}\text { Luminosity weighted } \\
\text { slope }\end{array}$ & $\begin{array}{r}\text { Mass-weighted } \\
\text { slope }\end{array}$ & $\begin{array}{r}\text { Luminosity weighted } \\
\text { slope }\end{array}$ \\
\hline IC 1256 & $0.25 \pm 0.05$ & $0.17 \pm 0.03$ & $-0.01 \pm 0.01$ & $-0.04 \pm 0.07$ \\
\hline IC 1683 & $-0.25 \pm 0.02$ & $-0.17 \pm 0.02$ & $-0.31 \pm 0.02$ & $-0.02 \pm 0.03$ \\
\hline NGC 0001 & $0.23 \pm 0.18$ & $0.01 \pm 0.05$ & $0.20 \pm 0.04$ & $-0.10 \pm 0.12$ \\
\hline NGC 0036 & $0.08 \pm 0.05$ & $0.02 \pm 0.07$ & $0.10 \pm 0.05$ & $-0.26 \pm 0.07$ \\
\hline NGC 0160 & $0.06 \pm 0.02$ & $0.02 \pm 0.01$ & $-0.17 \pm 0.02$ & $-0.19 \pm 0.03$ \\
\hline NGC 0214 & $0.16 \pm 0.10$ & $0.23 \pm 0.06$ & $-0.04 \pm 0.07$ & $0.07 \pm 0.16$ \\
\hline NGC 0234 & $-0.08 \pm 0.01$ & $0.81 \pm 0.01$ & $-0.08 \pm 0.01$ & $-0.08 \pm 0.01$ \\
\hline NGC 0257 & $-0.004 \pm 0.027$ & $0.02 \pm 0.01$ & $-0.14 \pm 0.02$ & $-0.23 \pm 0.05$ \\
\hline NGC 0776 & $0.00 \pm 0.02$ & $0.01 \pm 0.01$ & $0.15 \pm 0.03$ & $-0.05 \pm 0.03$ \\
\hline NGC 1167 & $0.02 \pm 0.10$ & $-0.03 \pm 0.13$ & $-0.17 \pm 0.04$ & $-0.34 \pm 0.07$ \\
\hline NGC 1645 & $0.02 \pm 0.08$ & $0.10 \pm 0.09$ & $-0.18 \pm 0.02$ & $-0.26 \pm 0.10$ \\
\hline NGC 2253 & $-0.03 \pm 0.01$ & $0.01 \pm 0.01$ & $-0.03 \pm 0.01$ & $-0.01 \pm 0.01$ \\
\hline NGC 2347 & $-0.10 \pm 0.03$ & $0.15 \pm 0.05$ & $-0.20 \pm 0.03$ & $-0.14 \pm 0.04$ \\
\hline NGC 2906 & $-0.14 \pm 0.01$ & $-0.14 \pm 0.01$ & $-0.011 \pm 0.005$ & $-0.14 \pm 0.01$ \\
\hline NGC 2916 & $-0.11 \pm 0.07$ & $0.06 \pm 0.09$ & $-0.05 \pm 0.08$ & $-0.38 \pm 0.05$ \\
\hline NGC 3106 & $0.11 \pm 0.02$ & $0.06 \pm 0.09$ & $-0.12 \pm 0.04$ & $-0.58 \pm 0.09$ \\
\hline NGC 3300 & $-0.06 \pm 0.01$ & $-0.04 \pm 0.01$ & $0.038 \pm 0.003$ & $0.029 \pm 0.004$ \\
\hline NGC 3614 & $-0.11 \pm 0.04$ & $-0.05 \pm 0.08$ & $-0.02 \pm 0.04$ & $-0.52 \pm 0.07$ \\
\hline NGC 3687 & $-0.21 \pm 0.02$ & $0.12 \pm 0.02$ & $-0.01 \pm 0.01$ & $0.15 \pm 0.02$ \\
\hline NGC 4047 & $-0.49 \pm 0.08$ & $-0.25 \pm 0.06$ & $0.37 \pm 0.04$ & $0.43 \pm 0.03$ \\
\hline NGC 4185 & $-0.16 \pm 0.06$ & $-0.24 \pm 0.05$ & $0.22 \pm 0.04$ & $-0.00 \pm 0.08$ \\
\hline NGC 4210 & $-0.16 \pm 0.02$ & $-0.15 \pm 0.01$ & $-0.09 \pm 0.02$ & $-0.04 \pm 0.03$ \\
\hline NGC 4470 & $0.19 \pm 0.02$ & $0.13 \pm 0.02$ & $-0.10 \pm 0.03$ & $0.01 \pm 0.02$ \\
\hline NGC 5000 & $0.08 \pm 0.45$ & $0.08 \pm 0.36$ & $-0.01 \pm 0.19$ & $0.04 \pm 0.14$ \\
\hline NGC 5016 & $-0.02 \pm 0.02$ & $0.07 \pm 0.01$ & $-0.13 \pm 0.06$ & $-0.17 \pm 0.04$ \\
\hline NGC 5205 & $-0.18 \pm 0.01$ & $-0.25 \pm 0.01$ & $0.09 \pm 0.01$ & $-0.06 \pm 0.02$ \\
\hline NGC 5218 & $-0.04 \pm 0.01$ & $-0.02 \pm 0.01$ & $0.17 \pm 0.02$ & $-0.03 \pm 0.01$ \\
\hline NGC 5378 & $-0.30 \pm 0.06$ & $-0.24 \pm 0.03$ & $-0.02 \pm 0.04$ & $-0.21 \pm 0.06$ \\
\hline NGC 5394 & $-0.02 \pm 0.01$ & $-0.02 \pm 0.01$ & $-0.03 \pm 0.02$ & $0.02 \pm 0.01$ \\
\hline NGC 5406 & $-0.02 \pm 0.01$ & $-0.02 \pm 0.01$ & $-0.01 \pm 0.02$ & $-0.09 \pm 0.02$ \\
\hline NGC 5614 & $0.14 \pm 0.04$ & $0.05 \pm 0.03$ & $0.07 \pm 0.04$ & $-0.02 \pm 0.06$ \\
\hline NGC 5633 & $-0.06 \pm 0.02$ & $-0.01 \pm 0.02$ & $0.08 \pm 0.03$ & $0.17 \pm 0.02$ \\
\hline NGC 5720 & $-0.14 \pm 0.09$ & $-0.20 \pm 0.09$ & $0.05 \pm 0.04$ & $-0.36 \pm 0.07$ \\
\hline NGC 5732 & $-0.21 \pm 0.08$ & $0.10 \pm 0.06$ & $0.21 \pm 0.11$ & $0.29 \pm 0.19$ \\
\hline NGC 5784 & $-0.117 \pm 0.005$ & $-0.09 \pm 0.03$ & $-0.08 \pm 0.02$ & $-0.08 \pm 0.02$ \\
\hline NGC 6004 & $-0.08 \pm 0.01$ & $-0.08 \pm 0.01$ & $0.0045 \pm 0.002$ & $0.033 \pm 0.005$ \\
\hline NGC 6063 & $-0.13 \pm 0.05$ & $-0.07 \pm 0.04$ & $0.05 \pm 0.02$ & $-0.16 \pm 0.03$ \\
\hline NGC 6154 & $-0.01 \pm 0.04$ & $-0.02 \pm 0.02$ & $-0.11 \pm 0.02$ & $-0.51 \pm 0.04$ \\
\hline NGC 6155 & $0.01 \pm 0.04$ & $-0.01 \pm 0.03$ & $0.06 \pm 0.01$ & $0.10 \pm 0.02$ \\
\hline NGC 6301 & $0.00 \pm 0.01$ & $0.02 \pm 0.01$ & $-0.05 \pm 0.01$ & $-0.16 \pm 0.01$ \\
\hline NGC 6497 & $0.01 \pm 0.02$ & $-0.16 \pm 0.02$ & $0.00 \pm 0.02$ & $-0.21 \pm 0.03$ \\
\hline NGC 6941 & $0.02 \pm 0.04$ & $0.01 \pm 0.02$ & $0.02 \pm 0.05$ & $-0.20 \pm 0.04$ \\
\hline NGC 7025 & $-0.20 \pm 0.07$ & $-0.23 \pm 0.08$ & $0.00 \pm 0.02$ & $-0.01 \pm 0.05$ \\
\hline NGC 7321 & $0.13 \pm 0.048$ & $0.22 \pm 0.03$ & $0.01 \pm 0.01$ & $-0.05 \pm 0.02$ \\
\hline NGC 7489 & $-0.50 \pm 0.11$ & $-0.21 \pm 0.09$ & $-0.29 \pm 0.04$ & $-0.58 \pm 0.07$ \\
\hline NGC 7549 & $-0.06 \pm 0.02$ & $-0.05 \pm 0.01$ & $-0.008 \pm 0.005$ & $0.03 \pm 0.01$ \\
\hline NGC 7563 & $-0.05 \pm 0.02$ & $-0.04 \pm 0.01$ & $0.026 \pm 0.006$ & $-0.01 \pm 0.01$ \\
\hline NGC 7591 & $-0.01 \pm 0.01$ & $0.04 \pm 0.01$ & $-0.02 \pm 0.02$ & $-0.08 \pm 0.01$ \\
\hline NGC 7653 & $-0.11 \pm 0.06$ & $0.04 \pm 0.01$ & $-0.10 \pm 0.01$ & $-0.27 \pm 0.01$ \\
\hline NGC 7671 & $-0.19 \pm 0.01$ & $-0.16 \pm 0.01$ & $0.07 \pm 0.07$ & $0.08 \pm 0.01$ \\
\hline NGC 7782 & $-0.03 \pm 0.03$ & $-0.04 \pm 0.02$ & $-0.06 \pm 0.02$ & $-0.15 \pm 0.06$ \\
\hline UGC 00005 & $-0.22 \pm 0.03$ & $-0.045 \pm 0.003$ & $0.03 \pm 0.01$ & $0.03 \pm 0.01$ \\
\hline UGC 00036 & $-0.27 \pm 0.08$ & $-0.26 \pm 0.03$ & $0.11 \pm 0.03$ & $-0.27 \pm 0.05$ \\
\hline UGC 03253 & $-0.10 \pm 0.05$ & $-0.21 \pm 0.06$ & $-0.09 \pm 0.08$ & $-0.46 \pm 0.06$ \\
\hline UGC 07012 & $0.06 \pm 0.18$ & $-0.11 \pm 0.19$ & $0.25 \pm 0.08$ & $-0.02 \pm 0.23$ \\
\hline UGC 08234 & $0.01 \pm 0.01$ & $0.004 \pm 0.004$ & $-0.06 \pm 0.02$ & $-0.03 \pm 0.01$ \\
\hline UGC 10205 & $-0.02 \pm 0.16$ & $-0.01 \pm 0.13$ & $0.13 \pm 0.12$ & $0.18 \pm 0.13$ \\
\hline UGC 11649 & $-0.43 \pm 0.19$ & $-0.47 \pm 0.18$ & $0.03 \pm 0.06$ & $-0.09 \pm 0.12$ \\
\hline UGC 11680 & $-0.07 \pm 0.24$ & $0.03 \pm 0.13$ & $-0.07 \pm 0.19$ & $0.12 \pm 0.32$ \\
\hline UGC 12224 & $0.00 \pm 0.05$ & $0.14 \pm 0.12$ & $-0.38 \pm 0.09$ & $-0.20 \pm 0.10$ \\
\hline UGC 12816 & $-0.05 \pm 0.01$ & $0.000 \pm 0.002$ & $-0.009 \pm 0.002$ & $-0.018 \pm 0.004$ \\
\hline
\end{tabular}


P. Sánchez-Blázquez et al.: Stellar populations in galaxy discs

Table 7. Luminosity- and mass-weighted $\log ($ age $)$ and metallicity gradient calculated as the difference of the metallicity and age between $1.5 r_{\text {eff }}$ and $r_{\text {disc } 0 \text {. }}$

\begin{tabular}{|c|c|c|c|c|}
\hline & \multicolumn{2}{|c|}{$\overline{\overline{[Z / H]}}$} & \multicolumn{2}{|c|}{ loge Age } \\
\hline & $\begin{array}{r}\text { Mass-weighted } \\
\text { grad }\end{array}$ & $\begin{array}{r}\text { Luminosity weighted } \\
\text { grad }\end{array}$ & $\begin{array}{r}\text { Mass-weighted } \\
\text { grad }\end{array}$ & $\begin{array}{r}\text { Luminosity weighted } \\
\text { grad }\end{array}$ \\
\hline IC 1256 & $0.08 \pm 0.05$ & $0.09 \pm 0.03$ & $-0.01 \pm 0.01$ & $-0.12 \pm 0.08$ \\
\hline IC 1683 & $-0.28 \pm 0.02$ & $-0.01 \pm 0.02$ & $-0.34 \pm 0.02$ & $-0.07 \pm 0.03$ \\
\hline NGC 0001 & $0.14 \pm 0.07$ & $-0.01 \pm 0.05$ & $0.05 \pm 0.04$ & $-0.09 \pm 0.12$ \\
\hline NGC 0036 & - & - & - & - \\
\hline NGC 0160 & - & - & - & - \\
\hline NGC 0214 & $0.08 \pm 0.10$ & $0.05 \pm 0.06$ & $-0.01 \pm 0.07$ & $-0.06 \pm 0.16$ \\
\hline NGC 0234 & $-0.22 \pm 0.01$ & $0.10 \pm 0.01$ & $-0.15 \pm 0.01$ & $-0.08 \pm 0.01$ \\
\hline NGC 0257 & $0.11 \pm 0.03$ & $0.07 \pm 0.014$ & $-0.09 \pm 0.02$ & $-0.20 \pm 0.05$ \\
\hline NGC 0776 & $0.01 \pm 0.02$ & $0.05 \pm 0.01$ & $-0.10 \pm 0.03$ & $-0.07 \pm 0.03$ \\
\hline NGC 1167 & - & - & - & - \\
\hline NGC 1645 & - & - & - & - \\
\hline NGC 2253 & $-0.01 \pm 0.03$ & $-0.07 \pm 0.03$ & $-0.01 \pm 0.01$ & $-0.14 \pm 0.01$ \\
\hline NGC 2347 & $-0.10 \pm 0.03$ & $0.06 \pm 0.04$ & $-0.07 \pm 0.03$ & $-0.06 \pm 0.04$ \\
\hline NGC 2906 & $-0.05 \pm 0.01$ & $-0.03 \pm 0.01$ & $0.005 \pm 0.005$ & $-0.057 \pm 0.007$ \\
\hline NGC 2916 & $-0.16 \pm 0.07$ & $-0.13 \pm 0.11$ & $-0.15 \pm 0.08$ & $-0.54 \pm 0.05$ \\
\hline NGC 3106 & - & - & - & - \\
\hline NGC 3300 & $-0.050 \pm 0.006$ & $-0.036 \pm 0.005$ & $0.018 \pm 0.003$ & $0.007 \pm 0.004$ \\
\hline NGC 3614 & - & - & - & - \\
\hline NGC 3687 & $-0.11 \pm 0.03$ & $0.01 \pm 0.02$ & $-0.10 \pm 0.01$ & $-0.20 \pm 0.02$ \\
\hline NGC 4047 & $-0.28 \pm 0.08$ & $-0.16 \pm 0.06$ & $0.28 \pm 0.04$ & $0.23 \pm 0.03$ \\
\hline NGC 4185 & $-0.10 \pm 0.06$ & $-0.17 \pm 0.05$ & $0.03 \pm 0.04$ & $-0.15 \pm 0.08$ \\
\hline NGC 4210 & $-0.15 \pm 0.02$ & $-0.15 \pm 0.01$ & $-0.07 \pm 0.02$ & $-0.32 \pm 0.03$ \\
\hline NGC 4470 & $0.14 \pm 0.02$ & $0.12 \pm 0.02$ & $-0.08 \pm 0.03$ & $0.03 \pm 0.02$ \\
\hline NGC 5000 & $-0.02 \pm 0.14$ & $0.10 \pm 0.07$ & $-0.20 \pm 0.11$ & $-0.15 \pm 0.19$ \\
\hline NGC 5016 & $0.01 \pm 0.02$ & $0.009 \pm 0.009$ & $-0.09 \pm 0.06$ & $-0.09 \pm 0.04$ \\
\hline NGC 5205 & $-0.164 \pm 0.005$ & $-0.04 \pm 0.01$ & $-0.01 \pm 0.01$ & $-0.23 \pm 0.02$ \\
\hline NGC 5218 & $-0.09 \pm 0.01$ & $-0.027 \pm 0.004$ & $-0.25 \pm 0.02$ & $-0.11 \pm 0.01$ \\
\hline NGC 5378 & $0.24 \pm 0.12$ & $0.01 \pm 0.06$ & $-0.77 \pm 0.04$ & $-0.42 \pm 0.08$ \\
\hline NGC 5394 & $-0.04 \pm 0.01$ & $-0.02 \pm 0.01$ & $-0.08 \pm 0.02$ & $-0.02 \pm 0.01$ \\
\hline NGC 5406 & $-0.07 \pm 0.01$ & $-0.09 \pm 0.01$ & $0.003 \pm 0.020$ & $-0.17 \pm 0.02$ \\
\hline NGC 5614 & $0.18 \pm 0.04$ & $0.24 \pm 0.03$ & $0.15 \pm 0.04$ & $-0.11 \pm 0.06$ \\
\hline NGC 5633 & $-0.07 \pm 0.02$ & $-0.02 \pm 0.02$ & $0.03 \pm 0.02$ & $0.09 \pm 0.02$ \\
\hline NGC 5720 & $-0.14 \pm 0.12$ & $-0.25 \pm 0.22$ & $0.02 \pm 0.05$ & $-0.37 \pm 0.07$ \\
\hline NGC 5732 & $-0.09 \pm 0.20$ & $0.04 \pm 0.13$ & $0.04 \pm 0.11$ & $0.18 \pm 0.19$ \\
\hline NGC 5784 & $-0.08 \pm 0.01$ & $-0.06 \pm 0.03$ & $0.10 \pm 0.02$ & $0.13 \pm 0.02$ \\
\hline NGC 6004 & $-0.09 \pm 0.01$ & $-0.091 \pm 0.004$ & $0.000 \pm 0.002$ & $-0.0652 \pm 0.0054$ \\
\hline NGC 6063 & $-0.18 \pm 0.05$ & $-0.16 \pm 0.04$ & $-0.05 \pm 0.02$ & $-0.31 \pm 0.03$ \\
\hline NGC 6154 & $-0.02 \pm 0.04$ & $-0.03 \pm 0.04$ & $-0.08 \pm 0.03$ & $-0.29 \pm 0.05$ \\
\hline NGC 6155 & $-0.05 \pm 0.04$ & $-0.001 \pm 0.026$ & $-0.04 \pm 0.01$ & $-0.01 \pm 0.02$ \\
\hline NGC 6301 & $0.028 \pm 0.009$ & $0.08 \pm 0.02$ & $-0.05 \pm 0.01$ & $-0.13 \pm 0.01$ \\
\hline NGC 6497 & $-0.00 \pm 0.02$ & $-0.07 \pm 0.02$ & $-0.04 \pm 0.02$ & $-0.15 \pm 0.03$ \\
\hline NGC 6941 & $-0.02 \pm 0.03$ & $-0.10 \pm 0.04$ & $0.03 \pm 0.05$ & $-0.35 \pm 0.05$ \\
\hline NGC 7025 & $-0.13 \pm 0.07$ & $-0.14 \pm 0.08$ & $-0.04 \pm 0.02$ & $-0.11 \pm 0.05$ \\
\hline NGC 7321 & $-0.21 \pm 0.05$ & $-0.21 \pm 0.03$ & $0.06 \pm 0.01$ & $-0.16 \pm 0.02$ \\
\hline NGC 7489 & $-0.60 \pm 0.07$ & $-0.24 \pm 0.09$ & $-0.37 \pm 0.04$ & $-0.63 \pm 0.07$ \\
\hline NGC 7549 & $0.01 \pm 0.02$ & $0.006 \pm 0.009$ & $0.004 \pm 0.005$ & $-0.01 \pm 0.01$ \\
\hline NGC 7563 & $-0.003 \pm 0.014$ & $0.00 \pm 0.01$ & $0.001 \pm 0.009$ & $-0.002 \pm 0.01$ \\
\hline NGC 7591 & $-0.039 \pm 0.013$ & $-0.015 \pm 0.013$ & $-0.015 \pm 0.021$ & $-0.045 \pm 0.013$ \\
\hline NGC 7653 & - & - & - & - \\
\hline NGC 7671 & $-0.18 \pm 0.01$ & $-0.15 \pm 0.01$ & $0.07 \pm 0.01$ & $0.08 \pm 0.01$ \\
\hline NGC 7782 & $-0.14 \pm 0.01$ & $-0.11 \pm 0.01$ & $-0.11 \pm 0.01$ & $-0.28 \pm 0.03$ \\
\hline UGC 00005 & $-0.17 \pm 0.03$ & $-0.033 \pm 0.003$ & $0.02 \pm 0.01$ & $0.01 \pm 0.01$ \\
\hline UGC 00036 & - & - & - & - \\
\hline UGC 03253 & - & - & - & - \\
\hline UGC 07012 & - & - & - & - \\
\hline UGC 08234 & $-0.02 \pm 0.01$ & $-0.008 \pm 0.004$ & $0.02 \pm 0.02$ & $0.01 \pm 0.01$ \\
\hline UGC 10205 & $-0.09 \pm 0.16$ & $0.00 \pm 0.13$ & $0.00 \pm 0.12$ & $0.00 \pm 0.13$ \\
\hline UGC 11649 & $-0.51 \pm 0.17$ & $-0.52 \pm 0.16$ & $0.03 \pm 0.05$ & $-0.26 \pm 0.04$ \\
\hline UGC 11680 & - & - & - & - \\
\hline UGC 12224 & - & - & - & - \\
\hline UGC 12816 & - & - & - & - \\
\hline
\end{tabular}




\section{Appendix A: Derivation of the age-metallicity relation}

One of the advantages of deriving star formation histories with STECKMAP is that, in principle, one can also derive the metallicity of the stellar population with different ages. However, due to the existent degeneracies in the determination of these parameters, one has to test if this is really possible. In Sánchez-Blázquez et al. (2011) - see their Appendix B - we already probed that we were able to recover the evolution of metallicity with time for the data with a similar $\mathrm{S} / \mathrm{N}$, resolution and wavelength coverage than that used in paper. In this appendix we repeat the test but using synthetic data with the characteristics of the CALIFA spectra. We have generated synthetic spectra for an exponentially declining star formation history $\mathrm{e}^{(-t / \tau)}$ with different values of $\tau, \tau=1$,
5, 10 and 20 Gyr and with two different chemical evolution, one with constant metallicity and one where the metallicity increase with age with the law $Z=0.2-($ age $(\mathrm{Gyr})-1.0) /(16.0)$.

We have used the models of V10 broadened to $150 \mathrm{~km} \mathrm{~s}^{-1}$ and we have added noise to simulate a spectrum of $S / N=40$. We then run STECKMAP on these spectra. Figure A.1 shows the simulated and the recovered age metallicity relation for the different synthetic spectra. The error bars in the figure are computed as the rms dispersion of the values obtained in 50 MonteCarlo simulations in which each pixel of the synthetic spectrum was modified randomly following a Gaussian distribution with a width given by the noise spectrum. As can be seen, although at all ages the metallicity is not very well constrained (the scatter from the different simulations is large), we can recover the age-metallicity relation in the synthetic spectra.
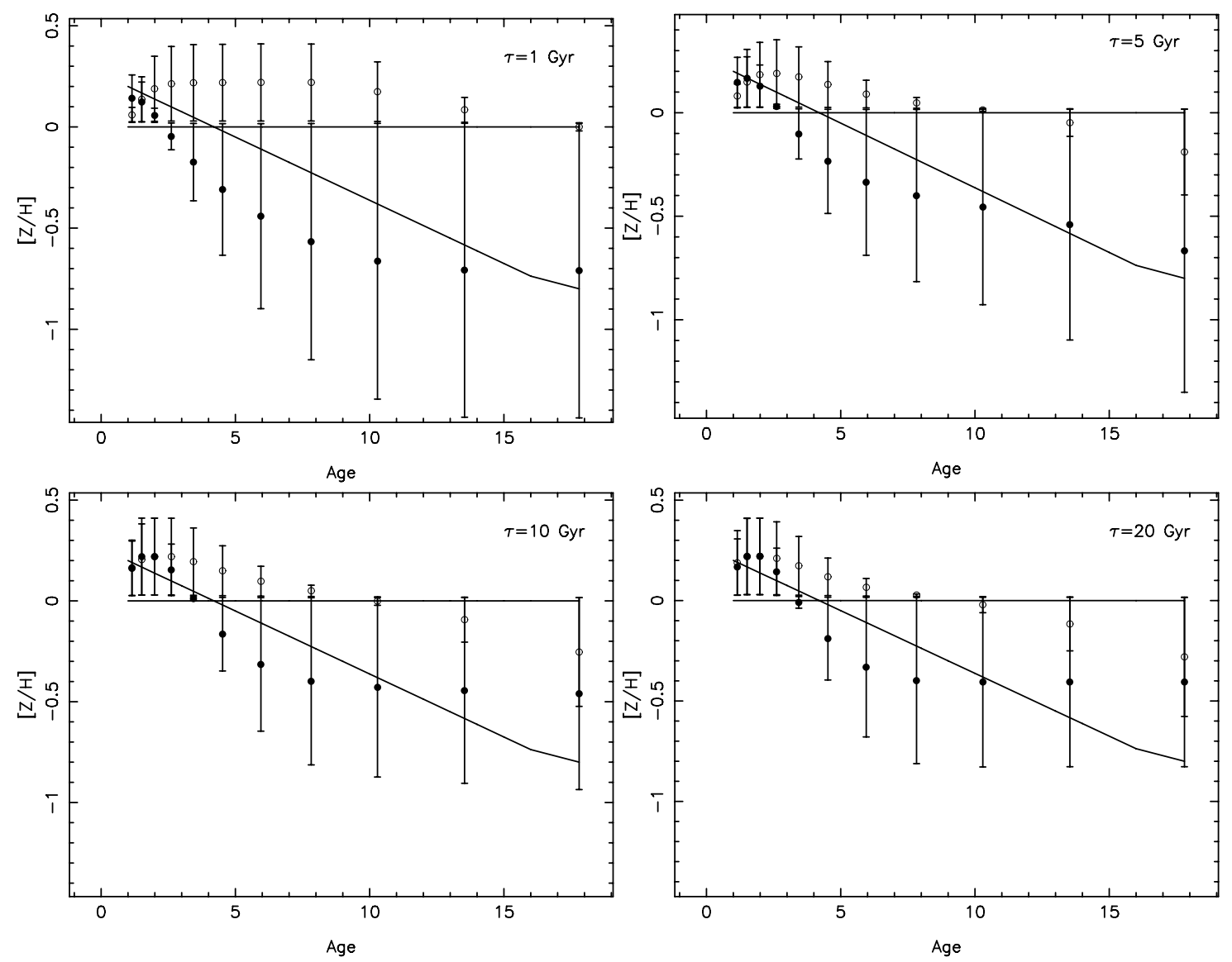

Fig. A.1. Age metallicity relation for a simulated exponential star formation history with different $\tau$ and with two different chemical evolution models. Solid lines indicate the simulated age-metallicity relation whilst the points show the recovered values. Open symbols shows the recovered age-metallicity relation in the case of constant metallicity with age whilst the filled symbols show the recovered age-metallicity relation in the case where the metallicity increase with time. Error bars represent the rms dispersion of a set of 50 Monte Carlo simulations where each pixel is moved according to a Gaussian distribution of width given by the errors. The synthetic spectra have been degraded to the resolution of the data and noise have been added to simulate a $\mathrm{S} / \mathrm{N}$ per $\AA$ of 40 . 


\section{Appendix B: Age gradients}

Figure B.1 shows the age gradients for our sample of galaxies.
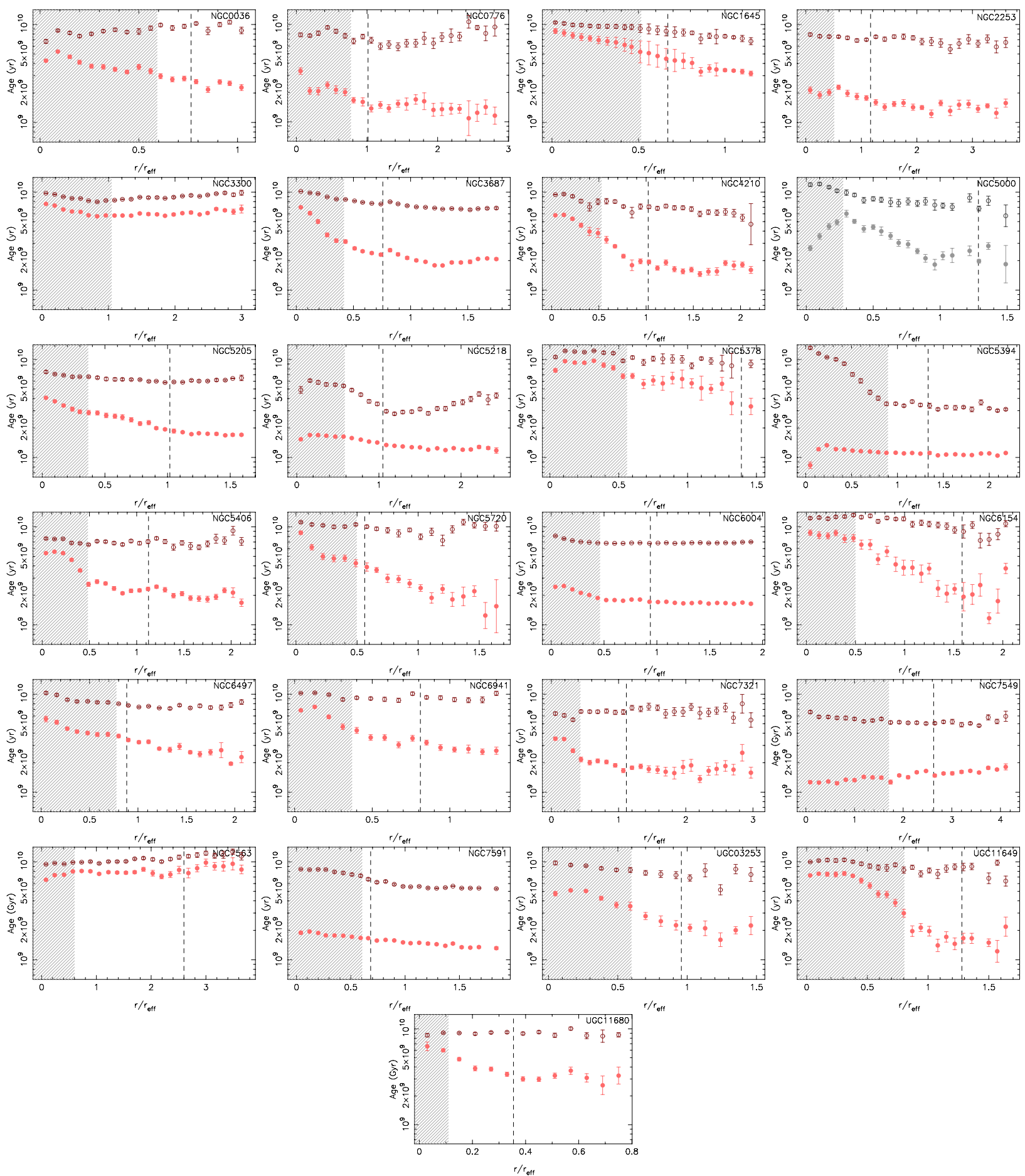

Fig. B.1. Mean luminosity- (light red) and mass- (dark red) age as a function of radius, normalised to the effective radius of the disc. Shaded area indicate the radial range of the surface brightness profile dominated by the bulge. The dashed line shows the bar length measured as indicated in the text. Solid lines shows the linear fit performed on the disc region. 
A\&A 570, A6 (2014)
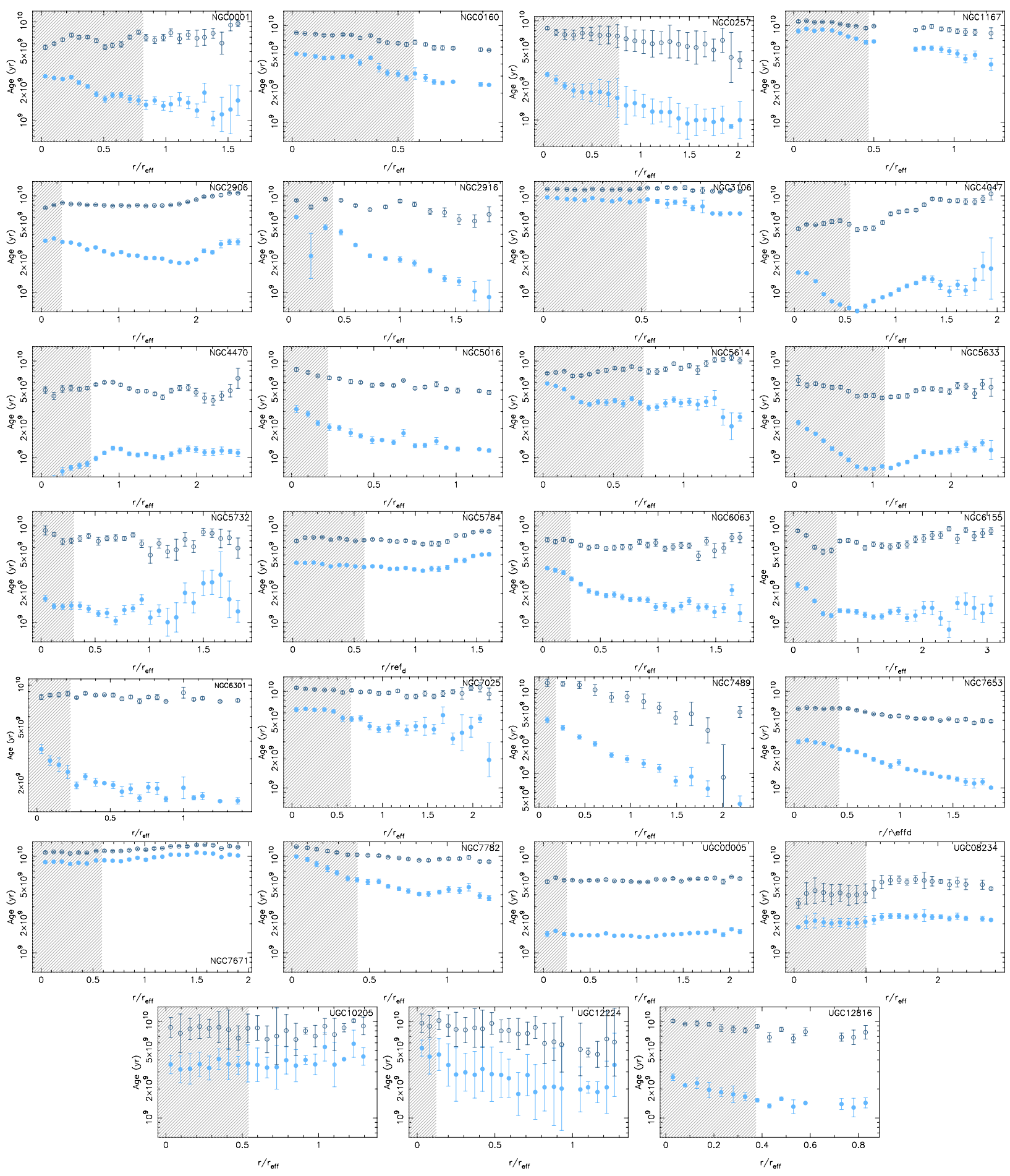

Fig. B.2. Age gradients of the sample of unbarred galaxies. 
P. Sánchez-Blázquez et al.: Stellar populations in galaxy discs
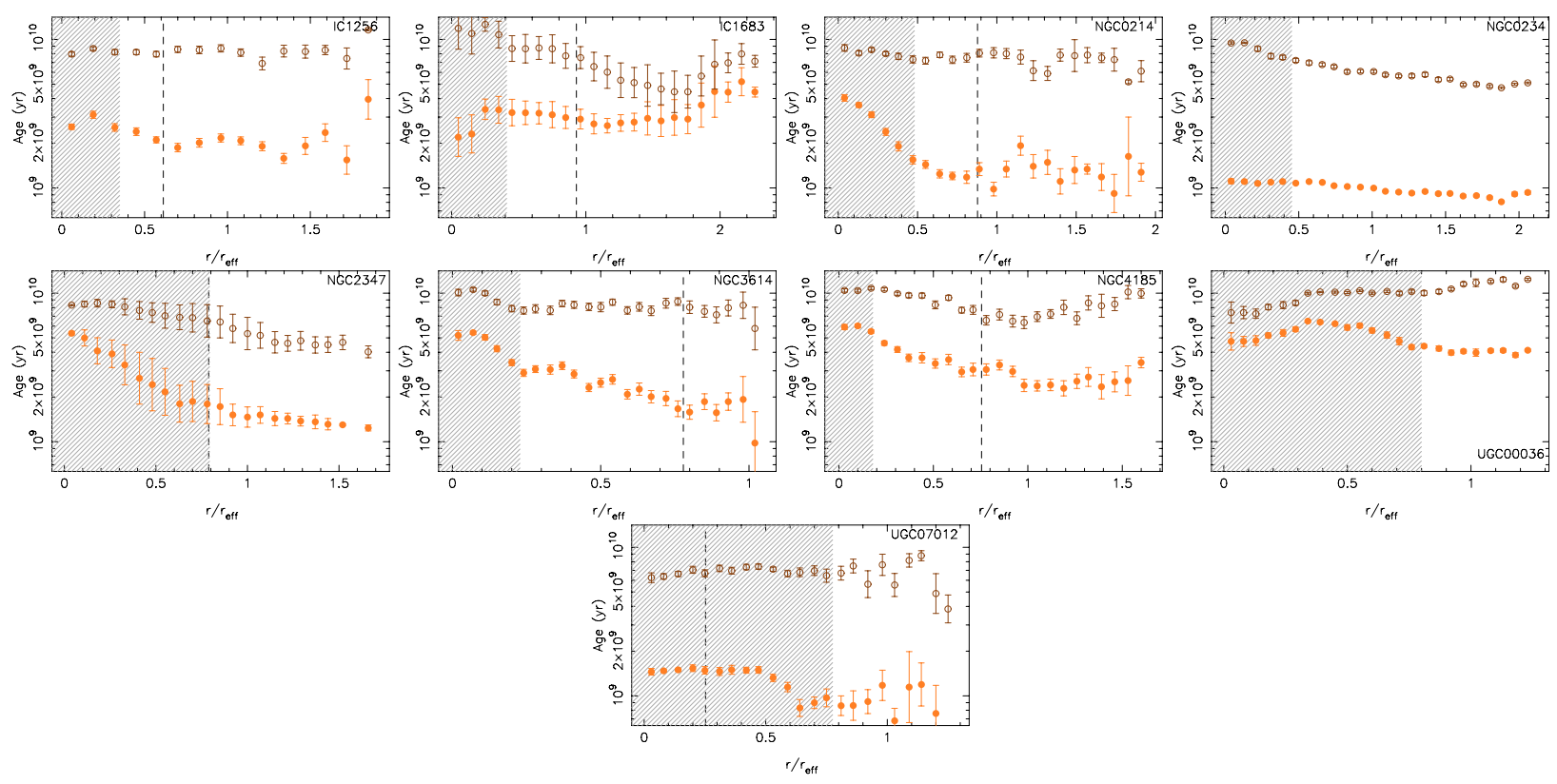

Fig. B.3. Age gradients of the sample of weakly barred galaxies. 


\section{Appendix C: Metallicity gradients}

Figure C.1 shows the metallicity gradients for our sample of galaxies.
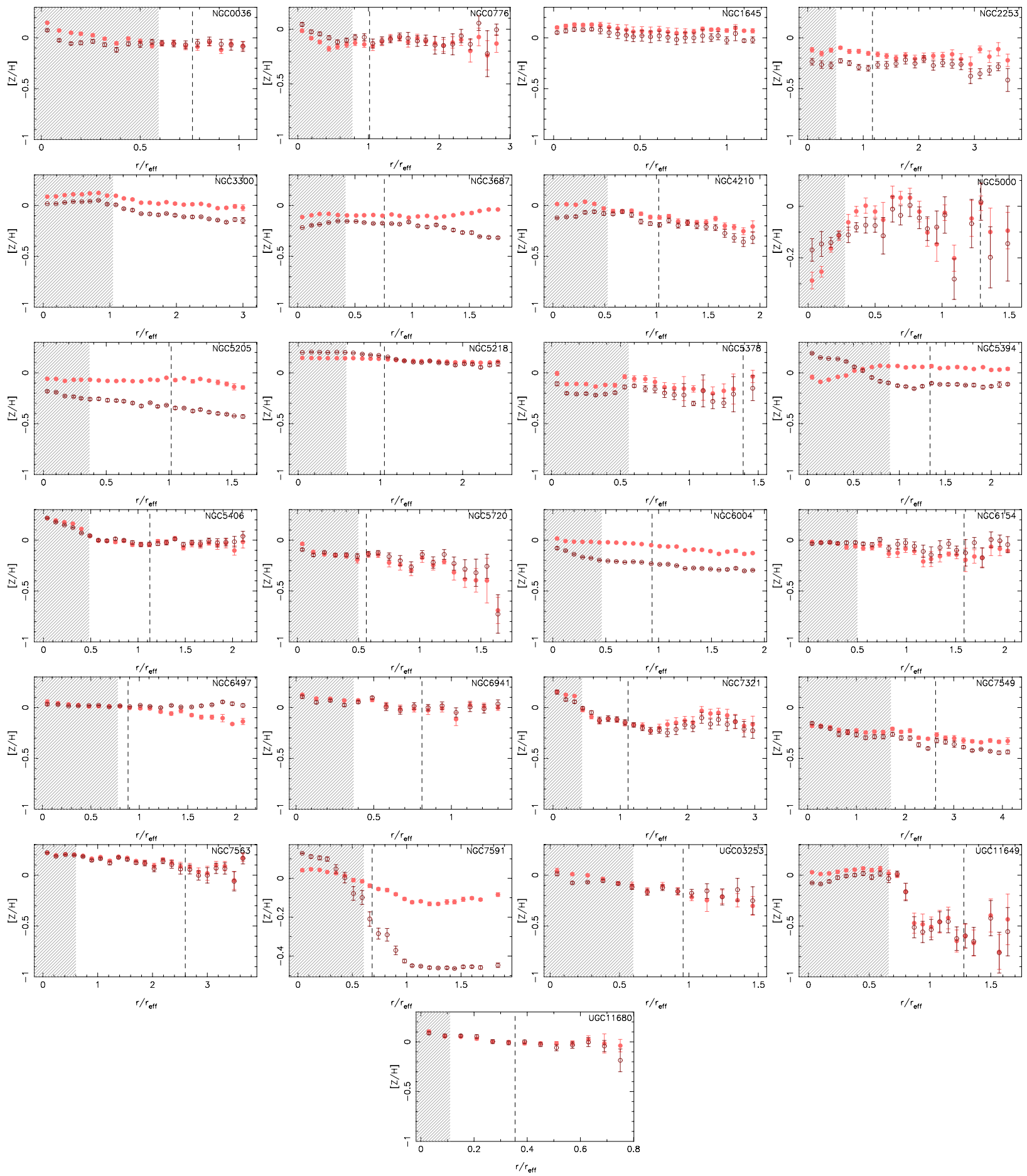

Fig. C.1. Luminosity- (light red) and mass- (dark red) metallicity gradients for our sample of barred galaxies. 
P. Sánchez-Blázquez et al.: Stellar populations in galaxy discs
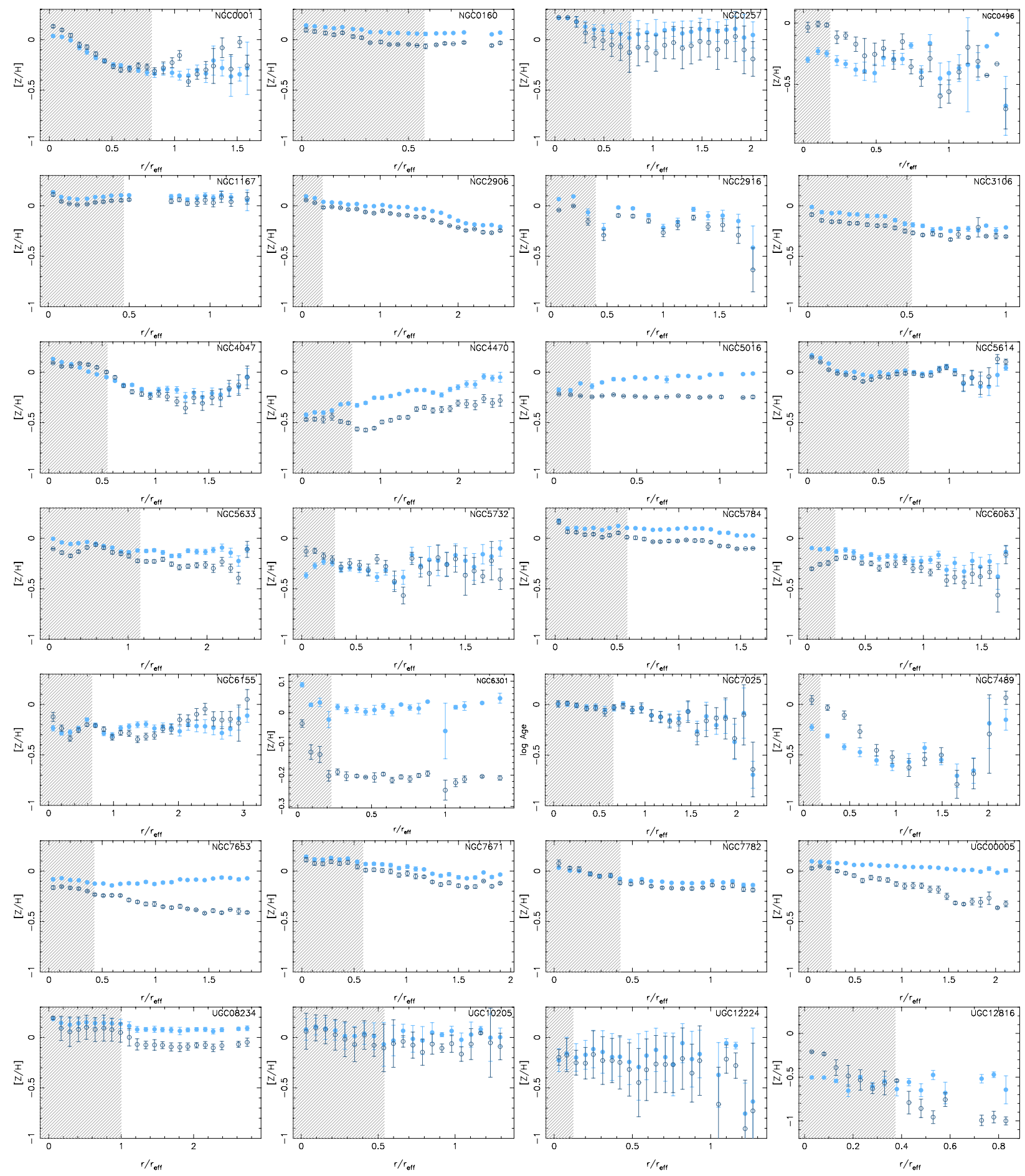

Fig. C.2. Luminosity- (light red) and mass- (dark red) metallicity gradients for our sample of barred galaxies. 
A\&A 570, A6 (2014)
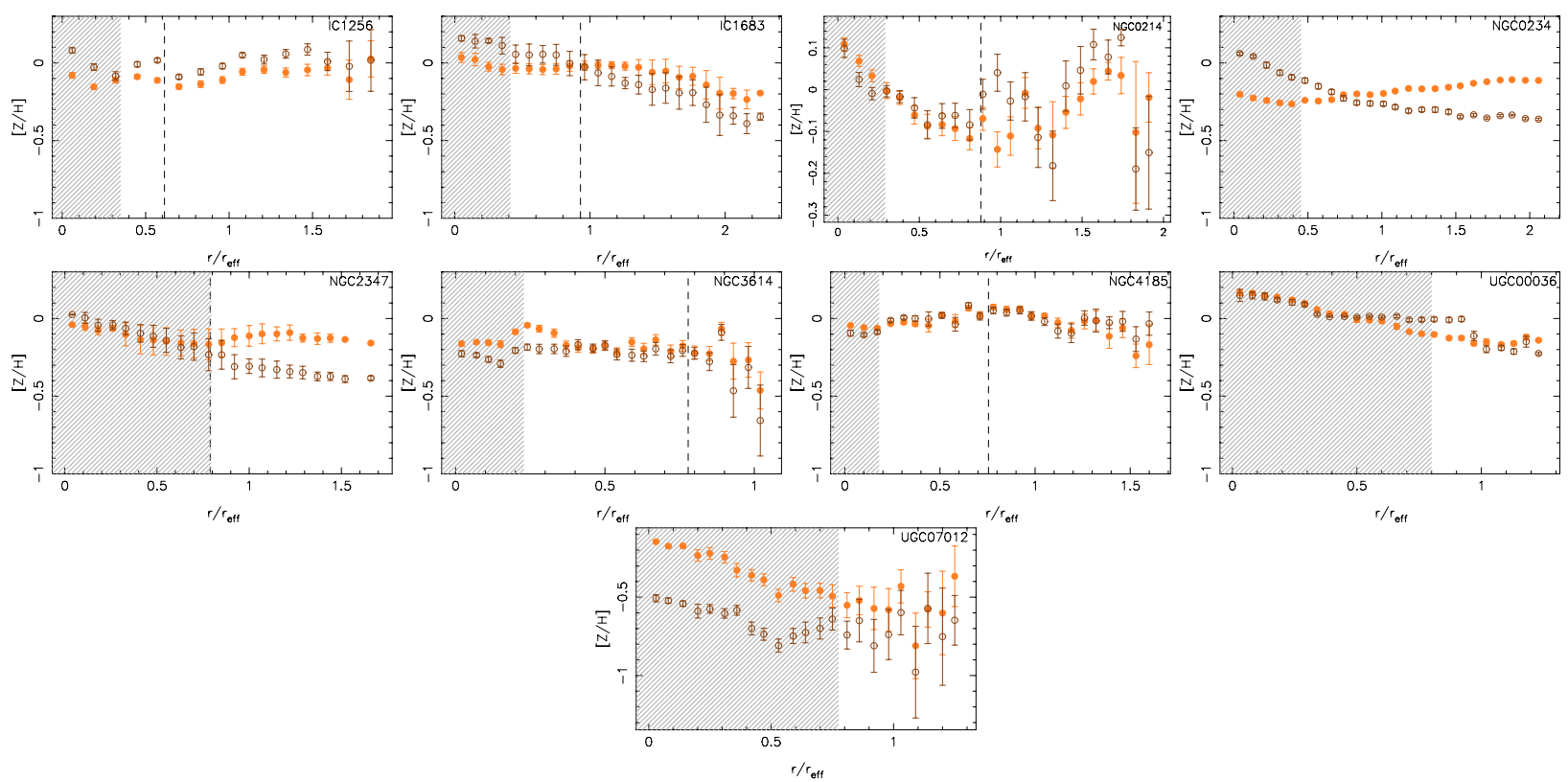

Fig. C.3. Light- (light yellow) and mass-weighted (dark yellow) metallicity gradient for the galaxies morphologically classified as weakly barred. 
P. Sánchez-Blázquez et al.: Stellar populations in galaxy discs
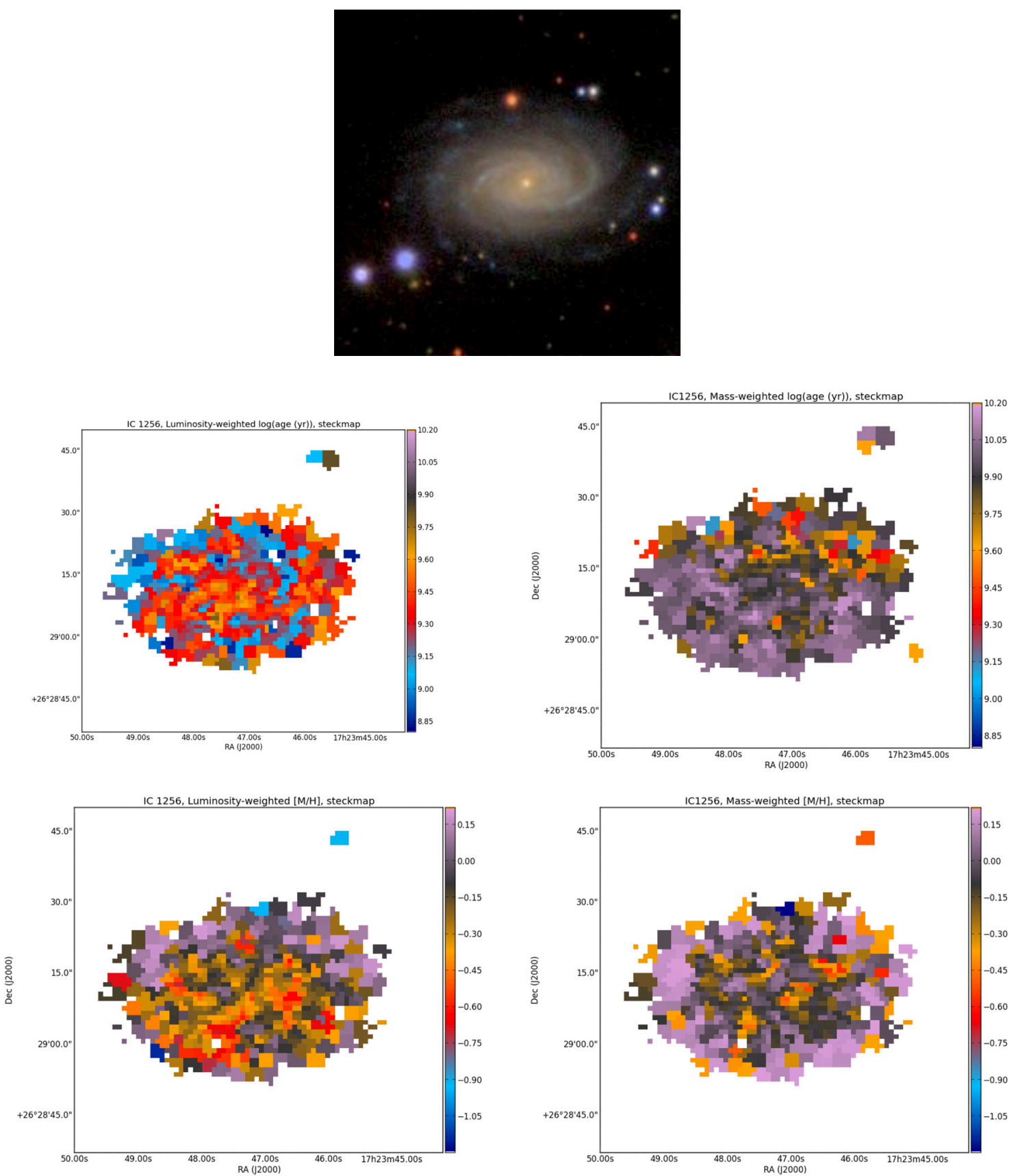

Fig. C.4. Mean $\log ($ age $)$ and metallicity maps for IC 1256. 
A\&A 570, A6 (2014)
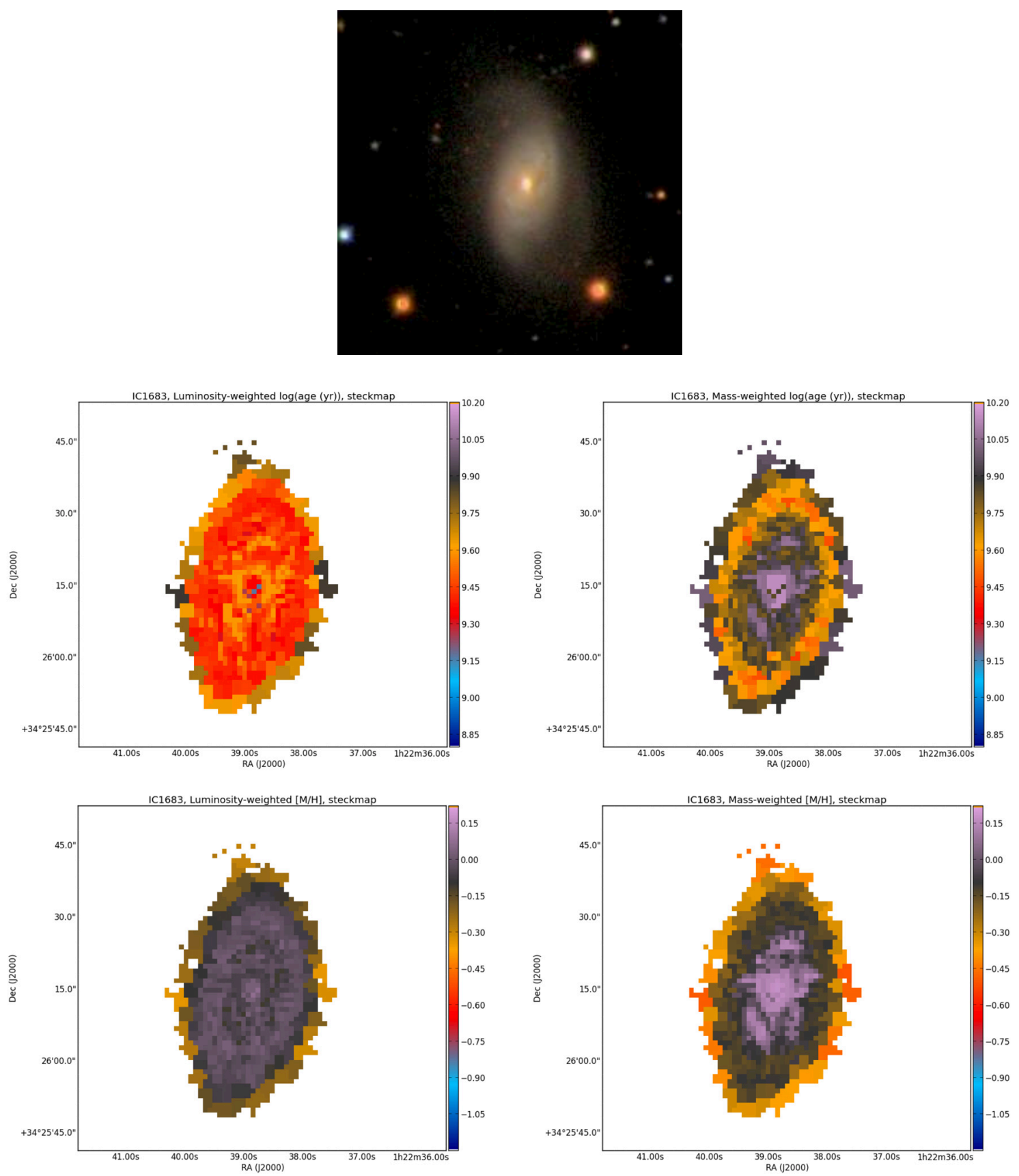

Fig. C.5. Mean log(age) and metallicity maps for IC1683. 
P. Sánchez-Blázquez et al.: Stellar populations in galaxy discs
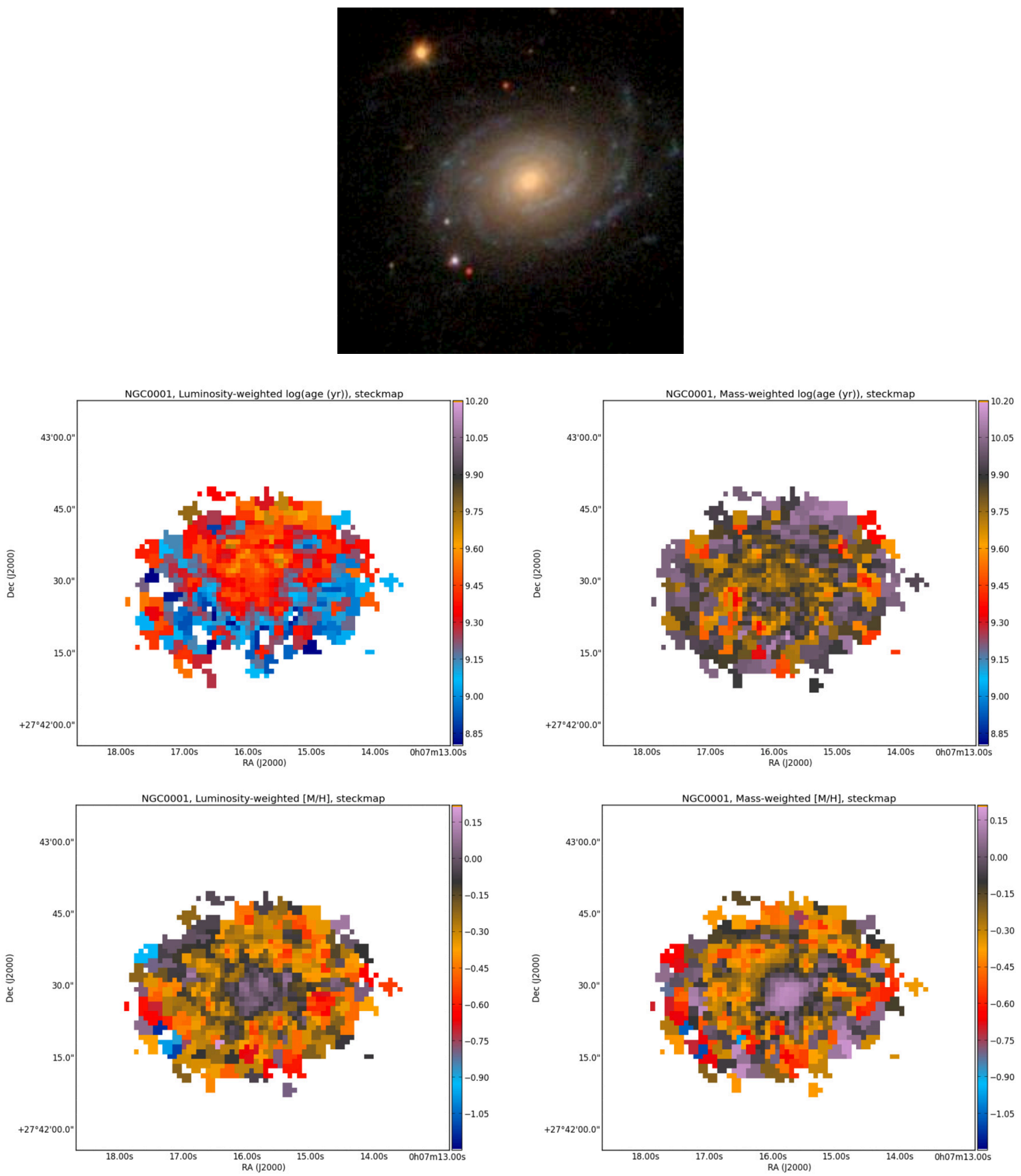

Fig. C.6. Mean $\log ($ age) and metallicity maps for NGC 0001. 
A\&A 570, A6 (2014)
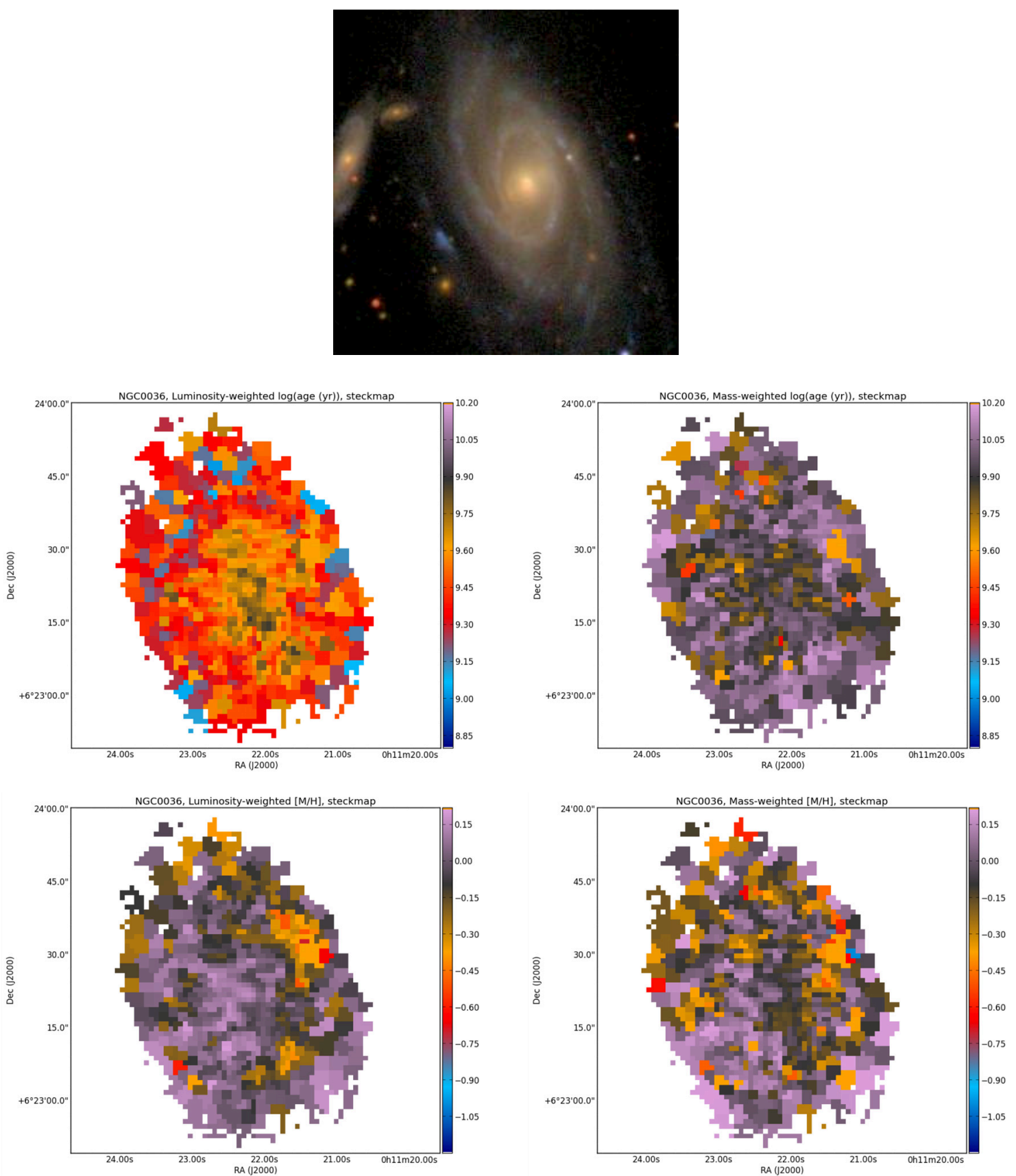

Fig. C.7. Mean log(age) and metallicity maps for NGC 0036. 
P. Sánchez-Blázquez et al.: Stellar populations in galaxy discs
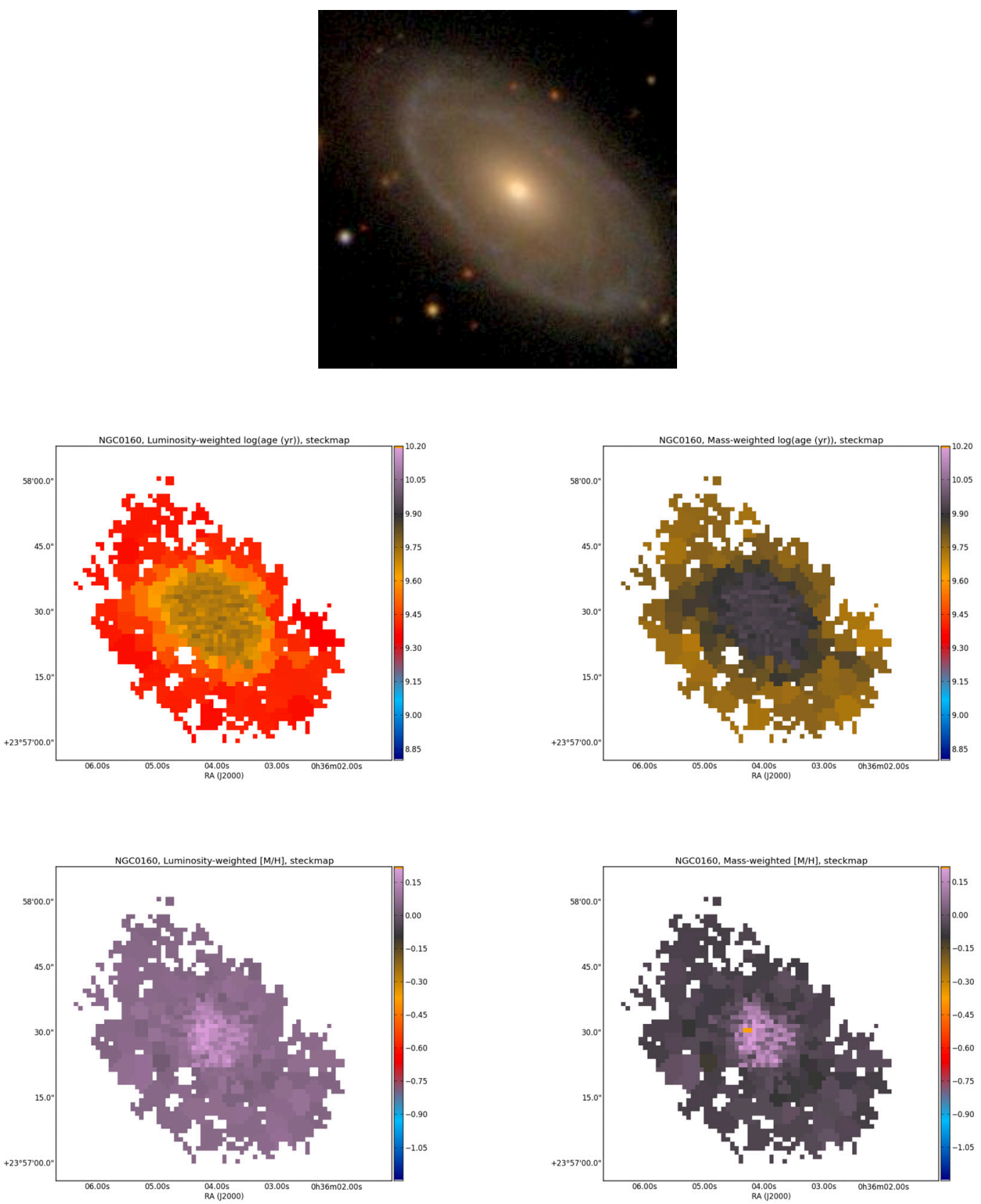

Fig. C.8. Mean $\log ($ age) and metallicity maps for NGC 0160 . 

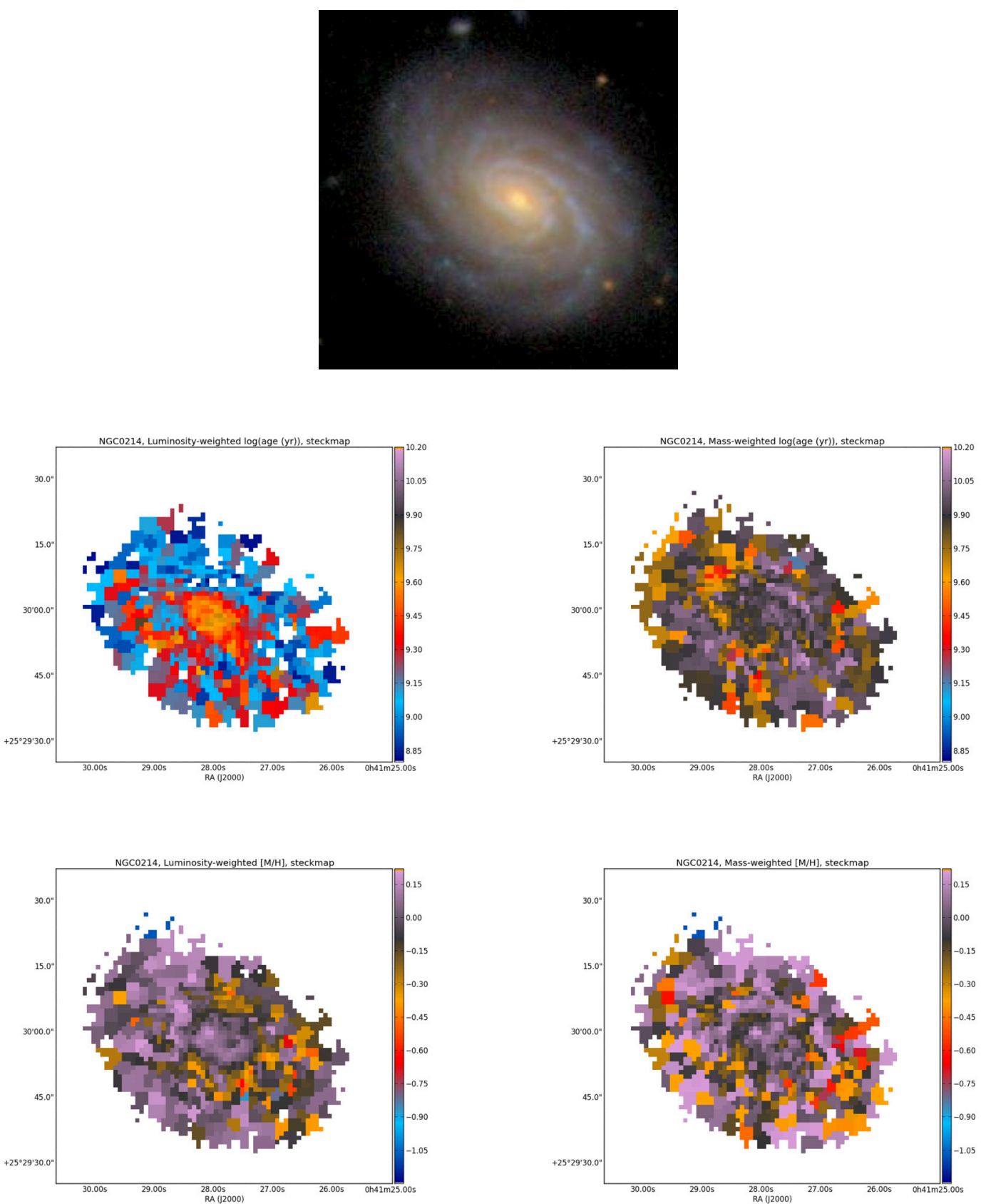

Fig. C.9. Mean $\log ($ age $)$ and metallicity maps for NGC 0214 . 
P. Sánchez-Blázquez et al.: Stellar populations in galaxy discs
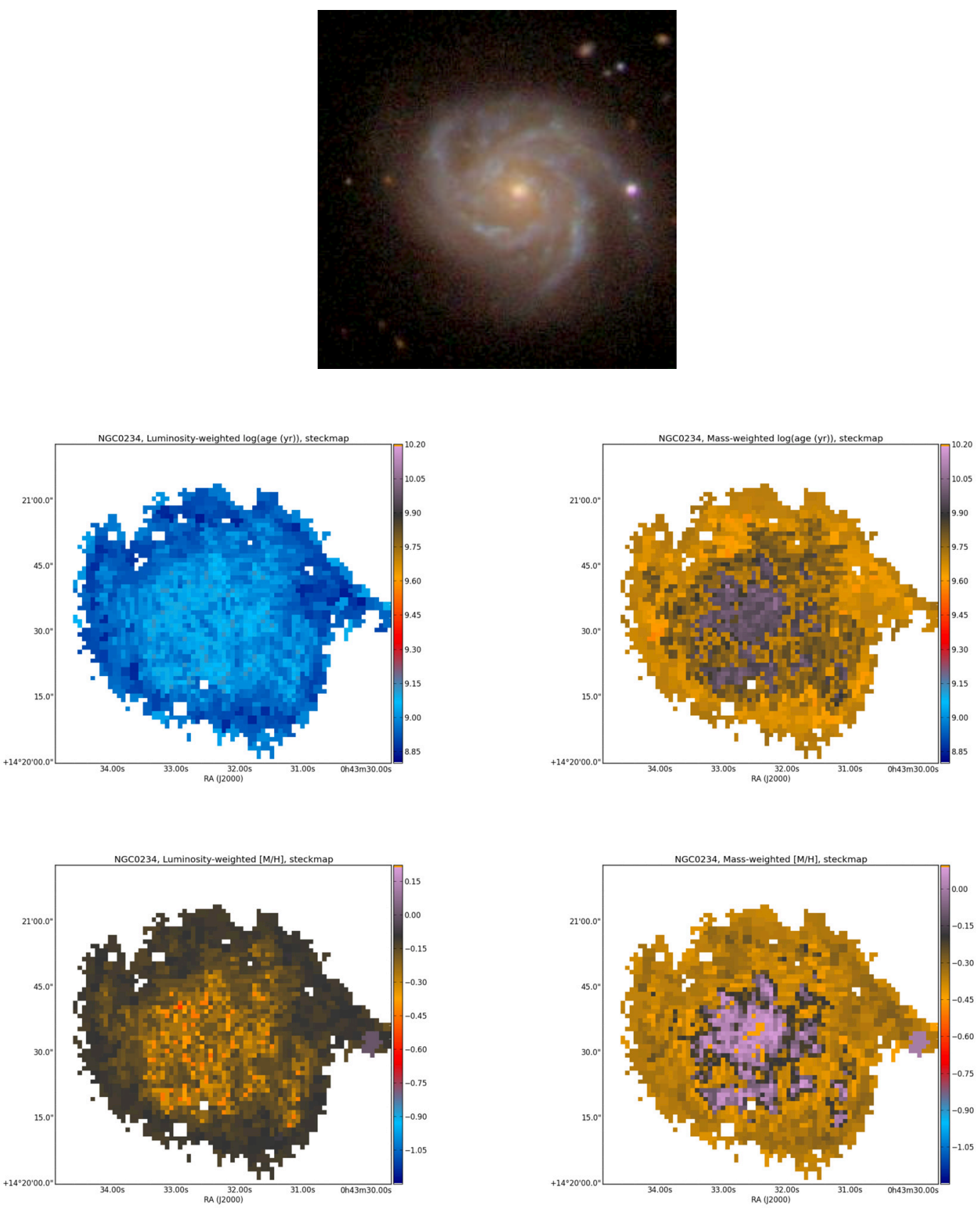

Fig. C.10. Mean log(age) and metallicity maps for NGC 0234. 
A\&A 570, A6 (2014)
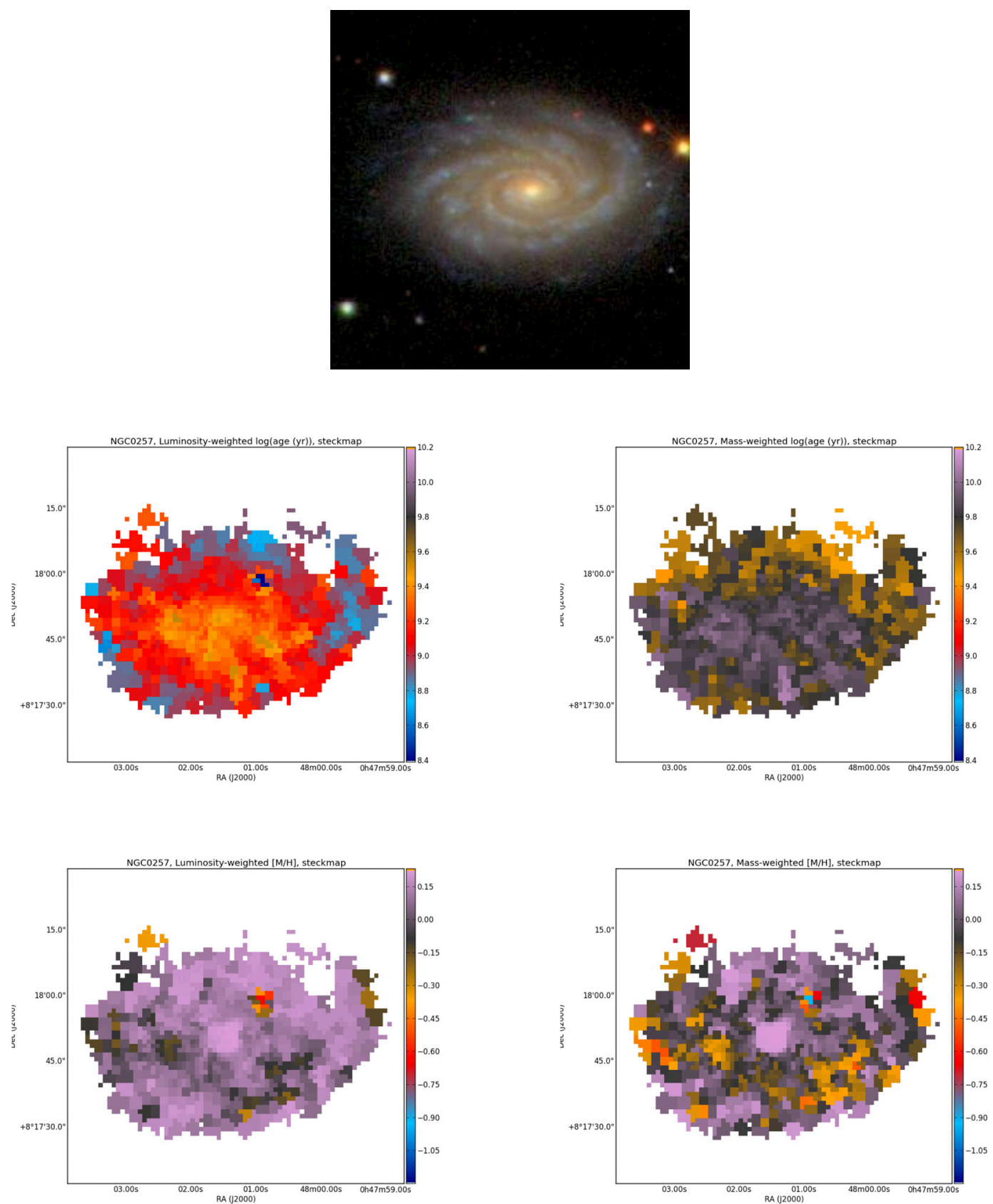

Fig. C.11. Mean log(age) and metallicity maps for NGC 0257. 
P. Sánchez-Blázquez et al.: Stellar populations in galaxy discs
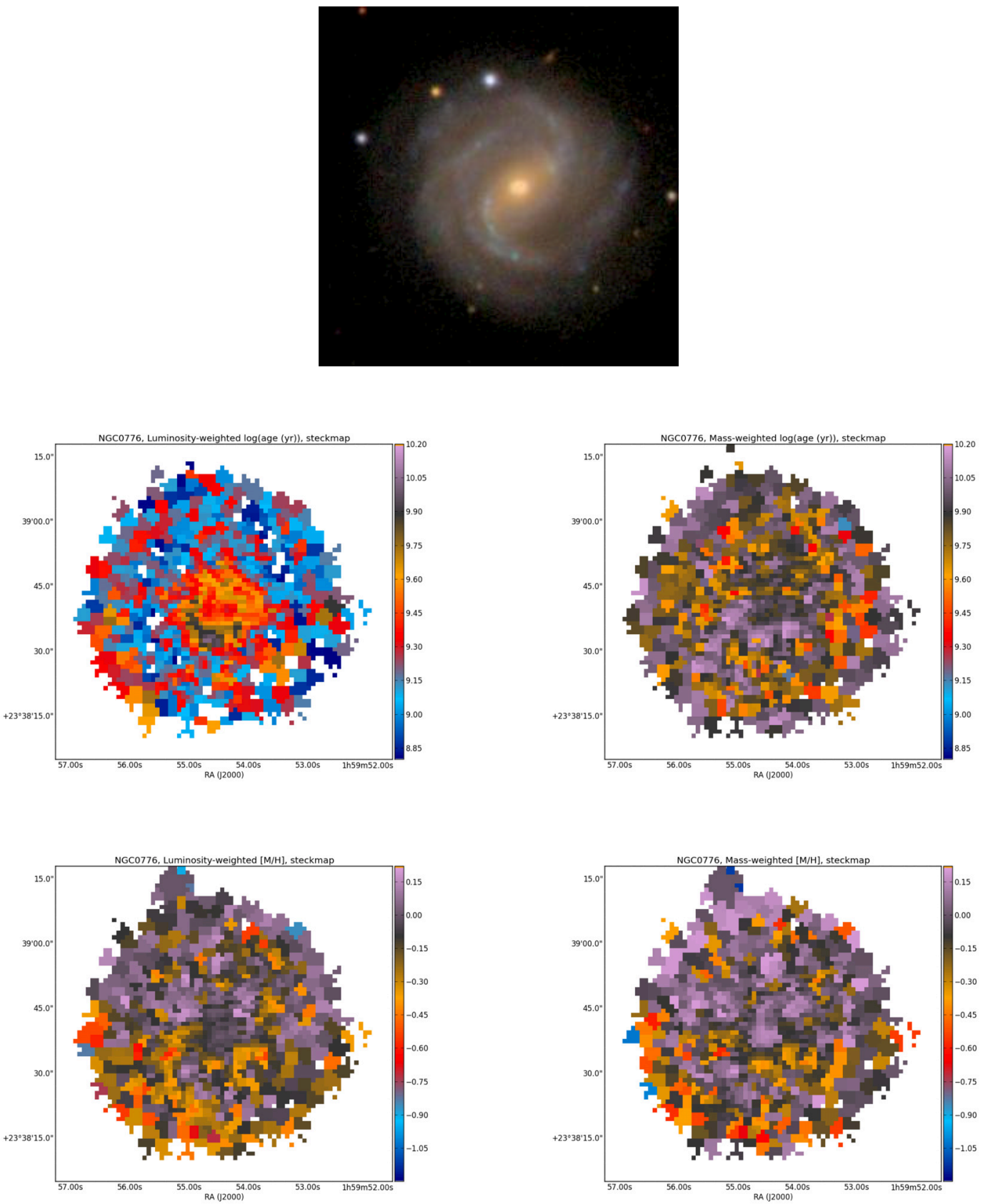

Fig. C.12. Mean log(age) and metallicity maps for NGC 0776. 
A\&A 570, A6 (2014)
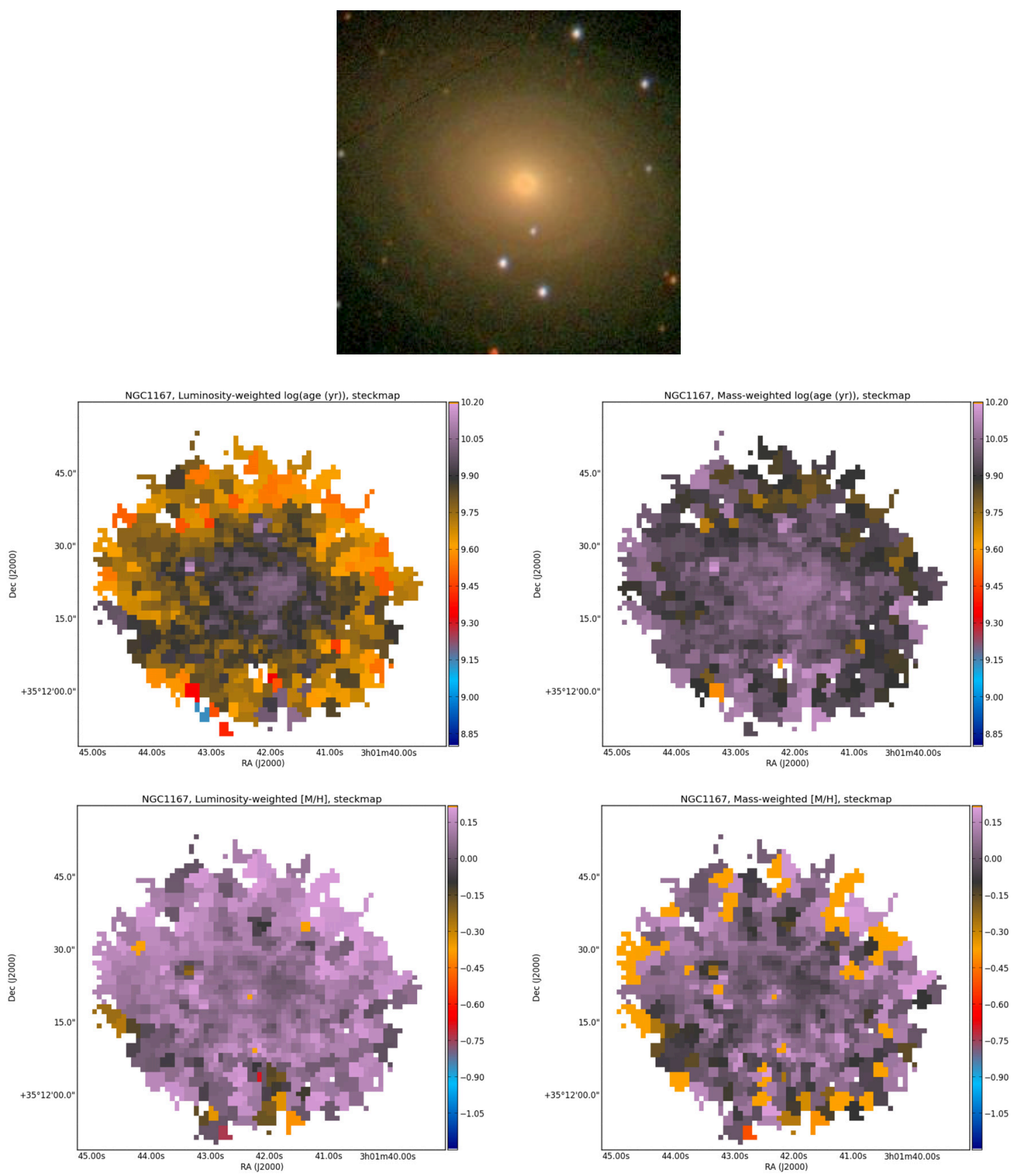

Fig. C.13. Mean log(age) and metallicity maps for NGC 1167. 
P. Sánchez-Blázquez et al.: Stellar populations in galaxy discs
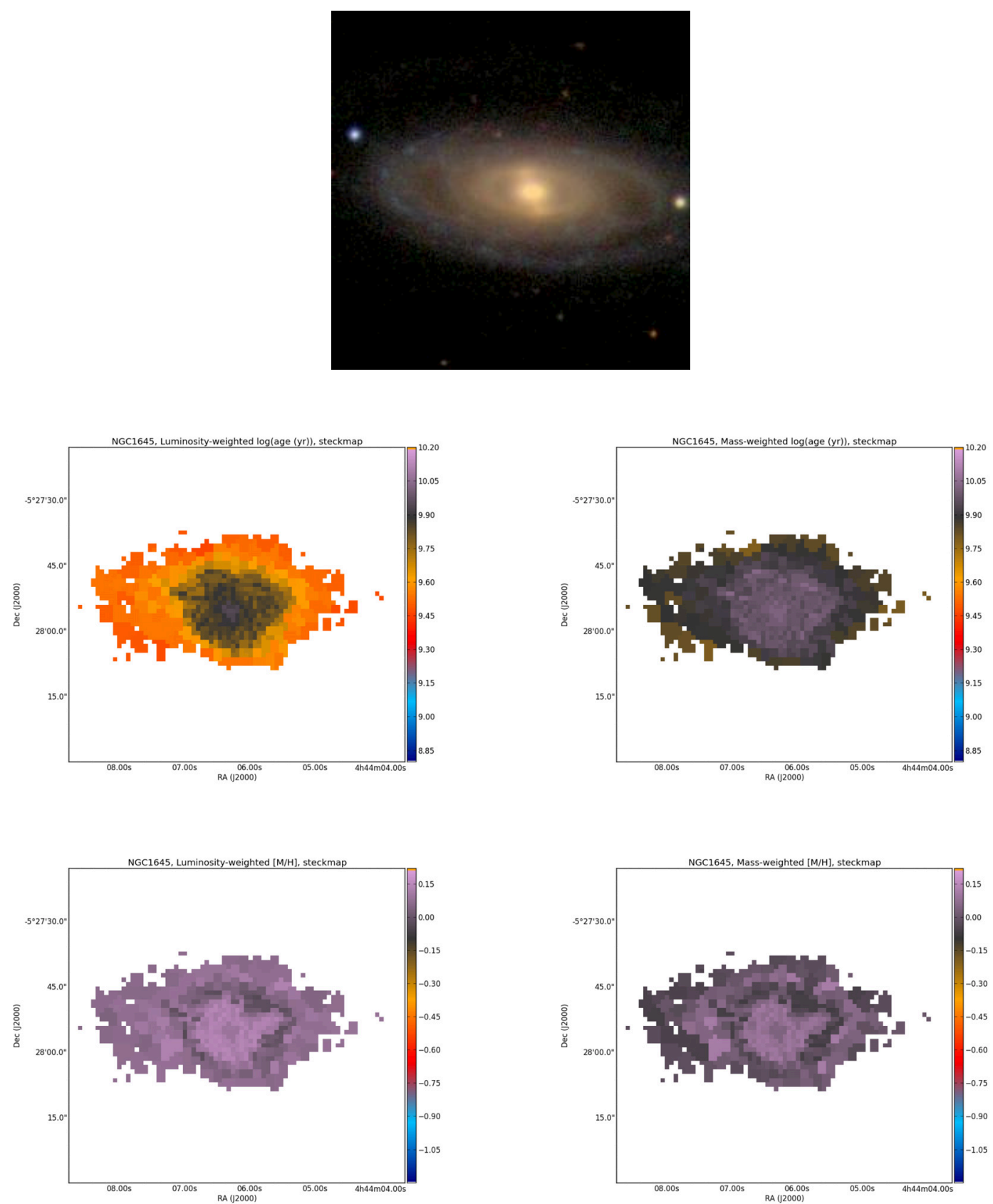

Fig. C.14. Mean log(age) and metallicity maps for NGC 1645. 
A\&A 570, A6 (2014)
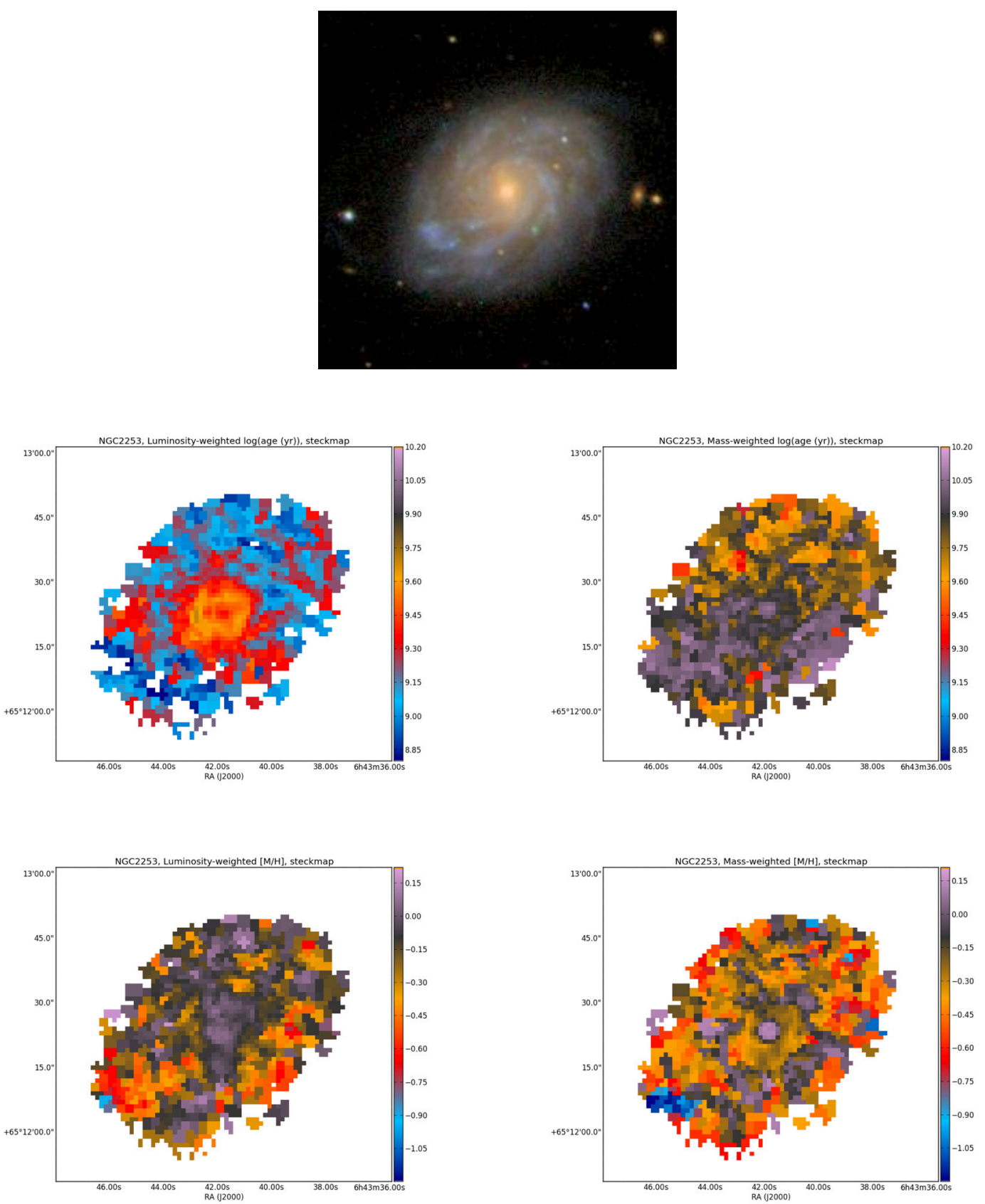

Fig. C.15. Mean log(age) and metallicity maps for NGC 2253. 
P. Sánchez-Blázquez et al.: Stellar populations in galaxy discs
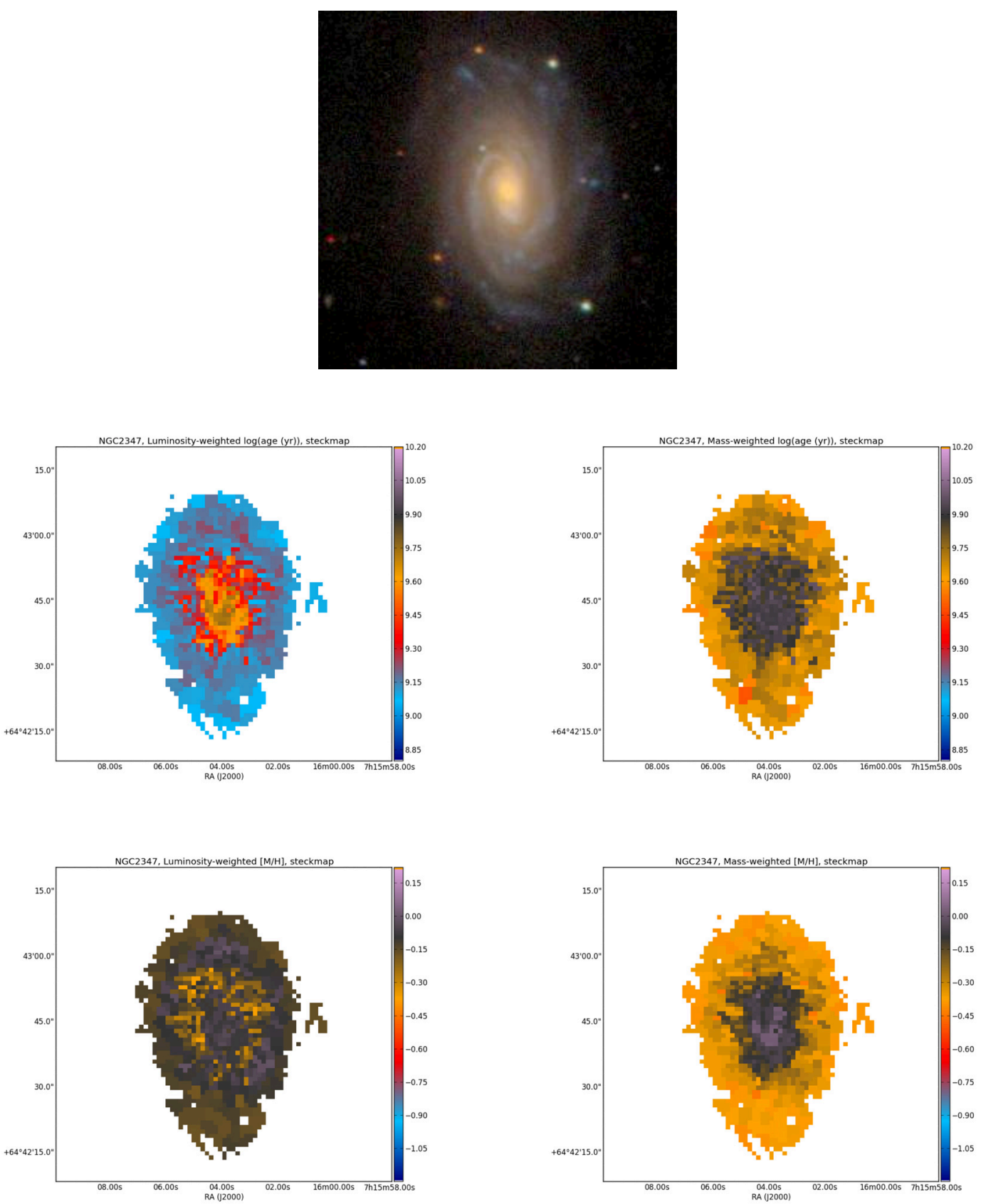

Fig. C.16. Mean log(age) and metallicity maps for NGC 2347. 

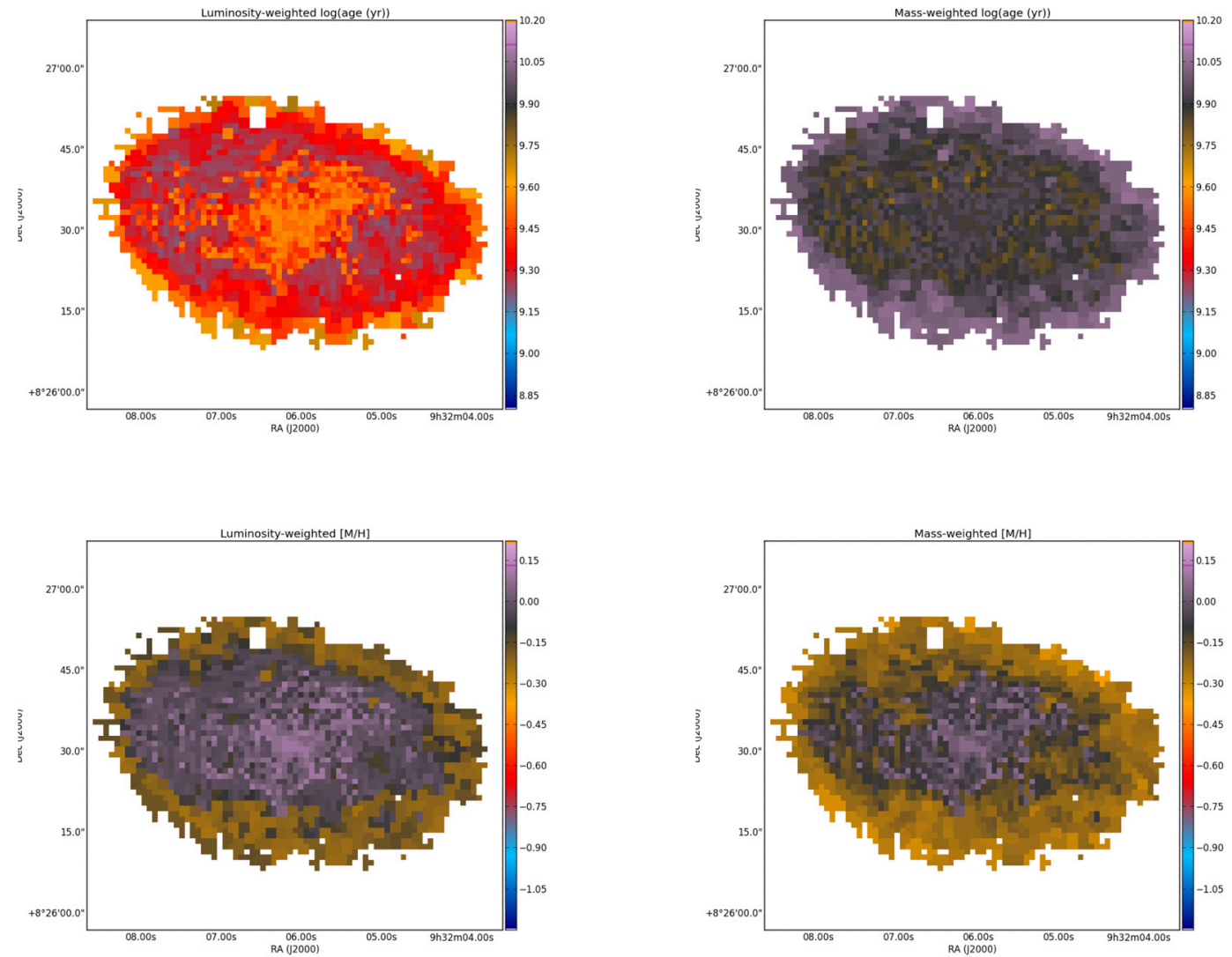

Fig. C.17. Mean log(age) and metallicity maps for NGC 2906. 
P. Sánchez-Blázquez et al.: Stellar populations in galaxy discs
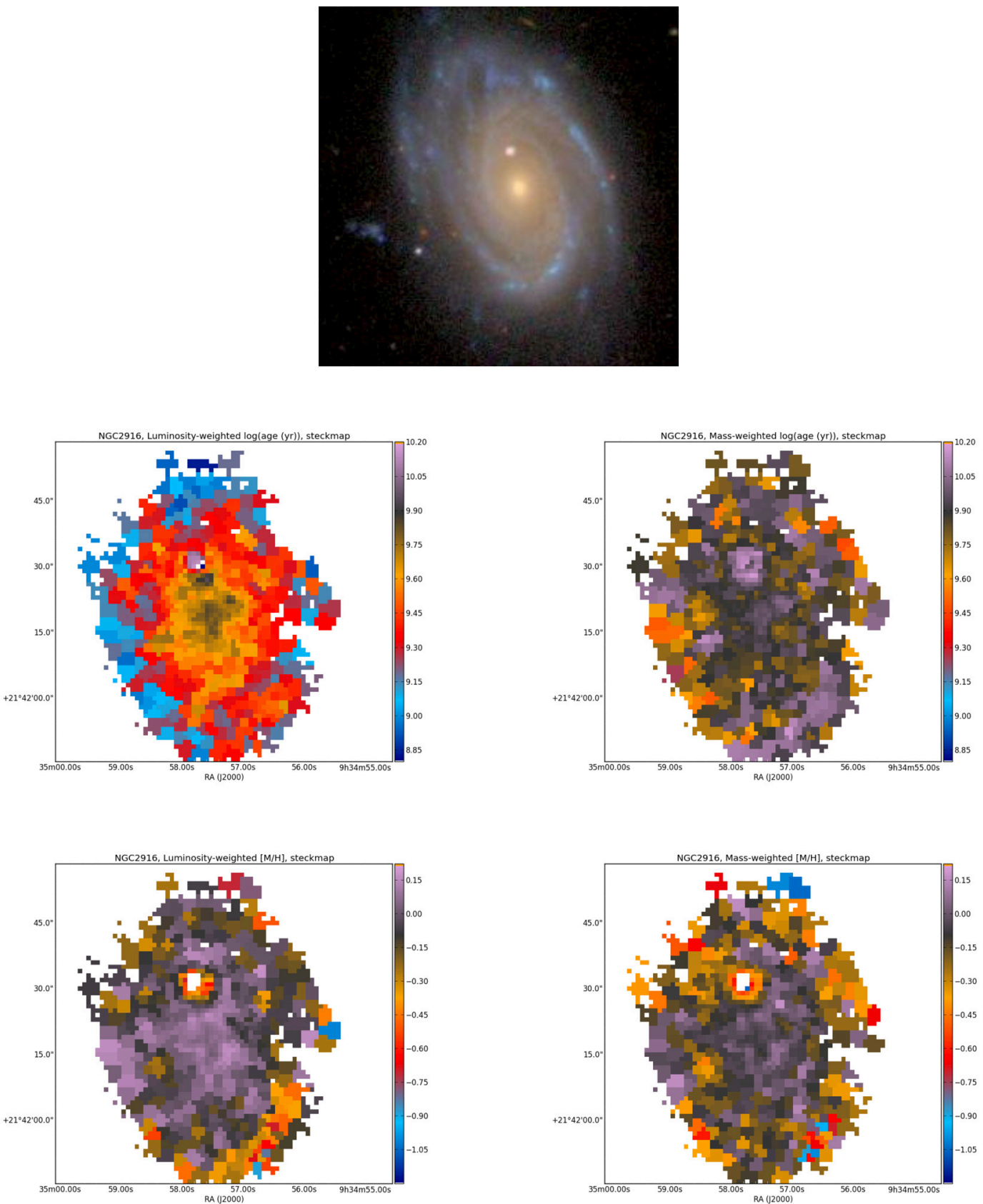

Fig. C.18. Mean log(age) and metallicity maps for NGC 2916. 

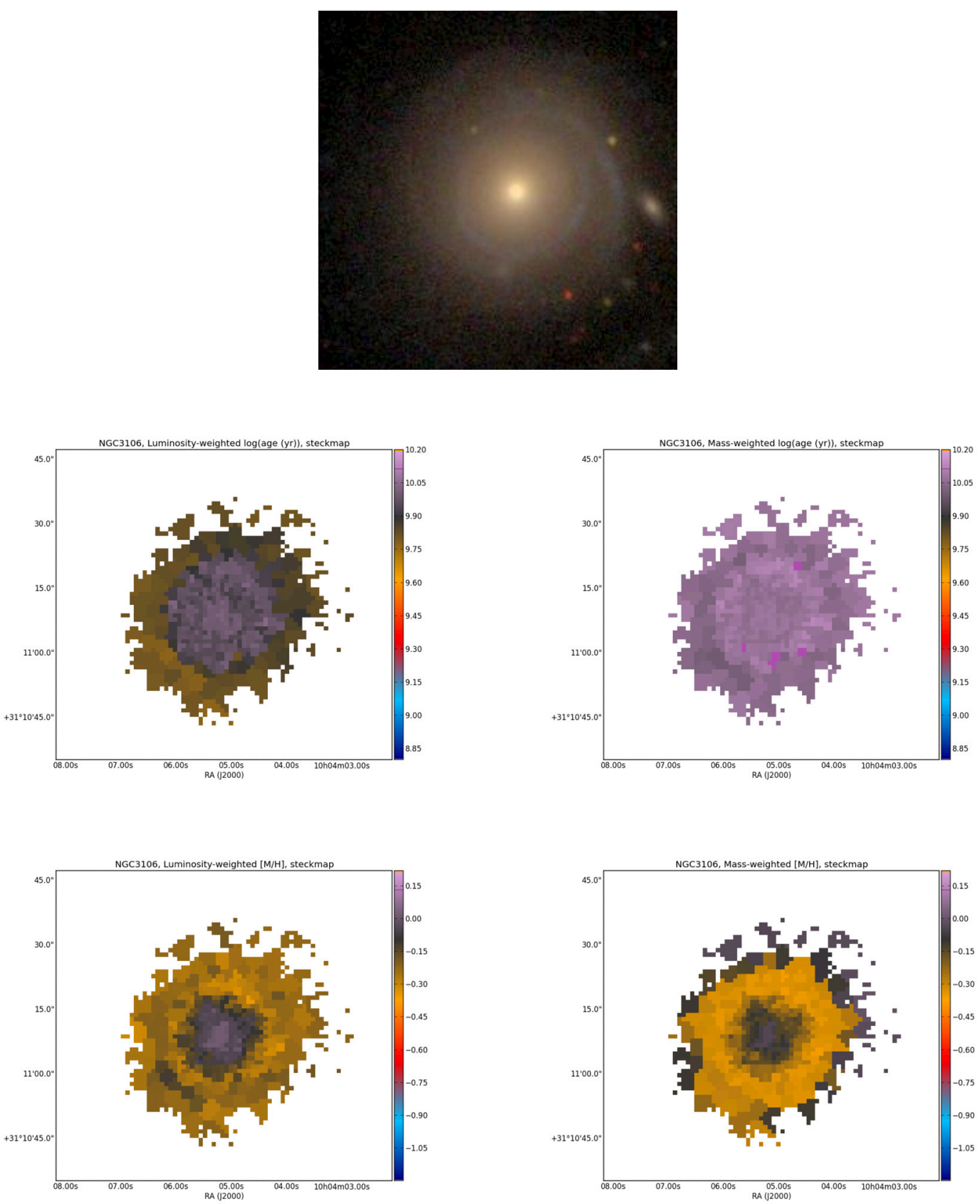

Fig. C.19. Mean log(age) and metallicity maps for NGC 3106. 
P. Sánchez-Blázquez et al.: Stellar populations in galaxy discs
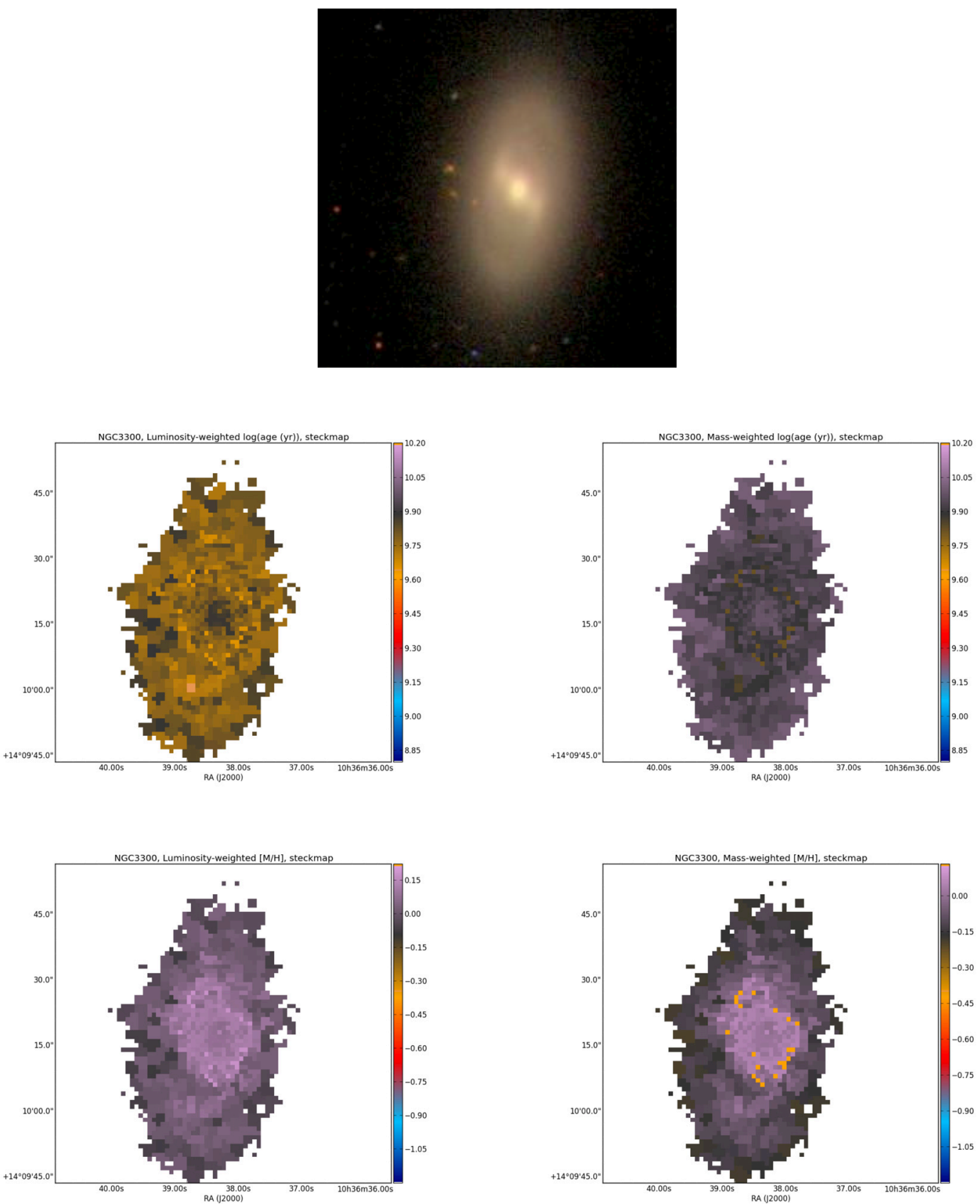

Fig. C.20. Mean log(age) and metallicity maps for NGC 3300 . 
A\&A 570, A6 (2014)
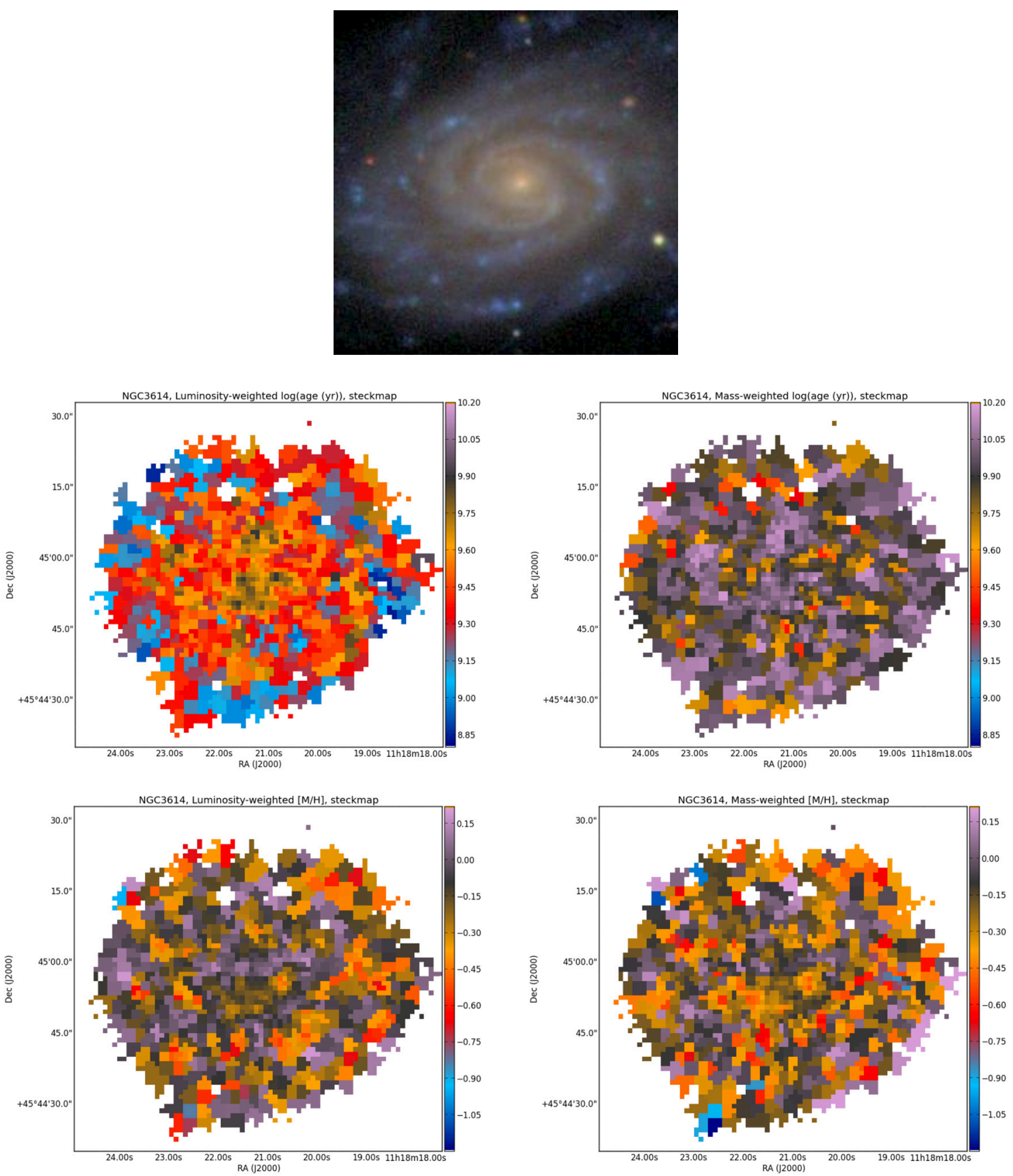

Fig. C.21. Mean log(age) and metallicity maps for NGC 3614 . 
P. Sánchez-Blázquez et al.: Stellar populations in galaxy discs
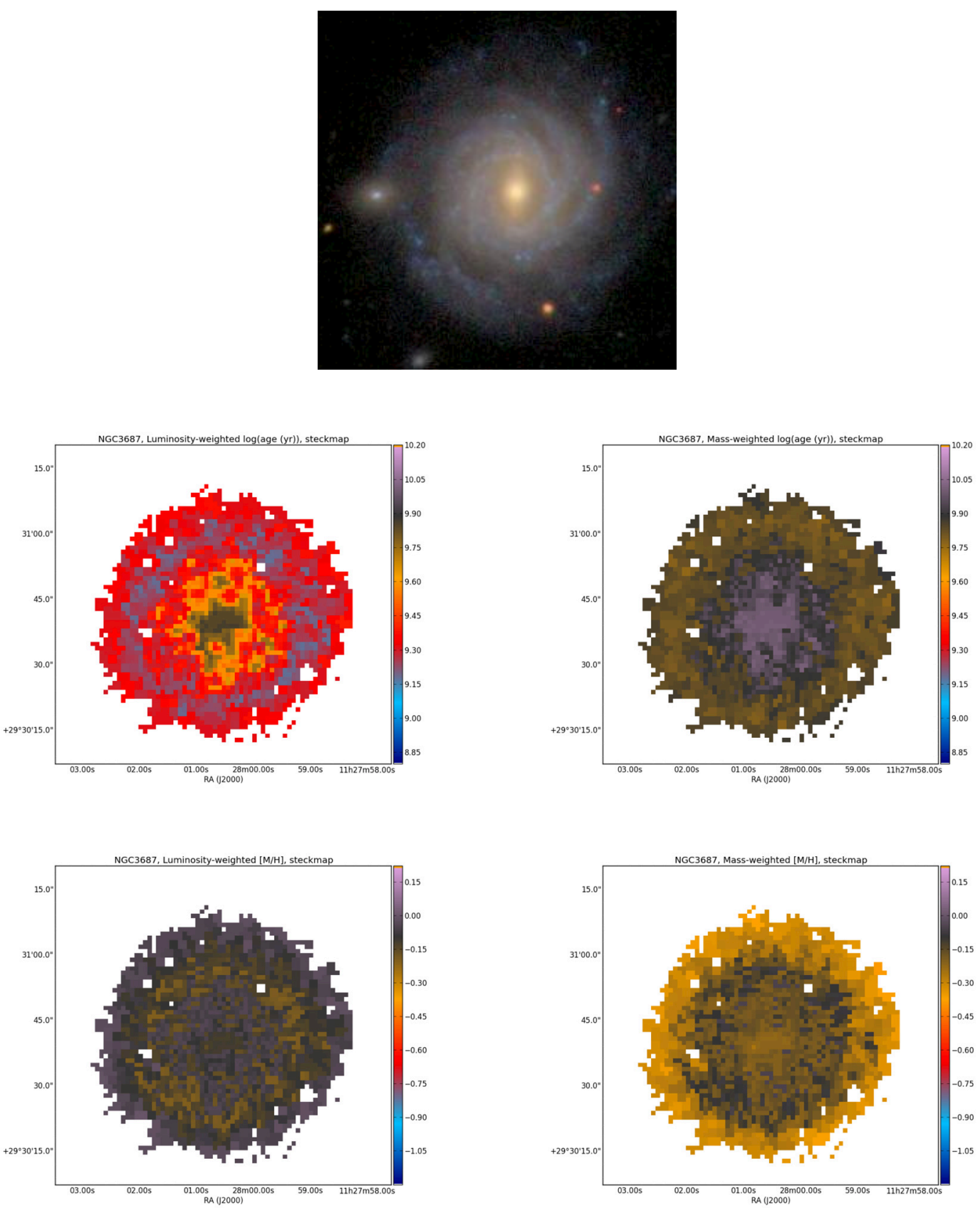

Fig. C.22. Mean log(age) and metallicity maps for NGC 3687 . 

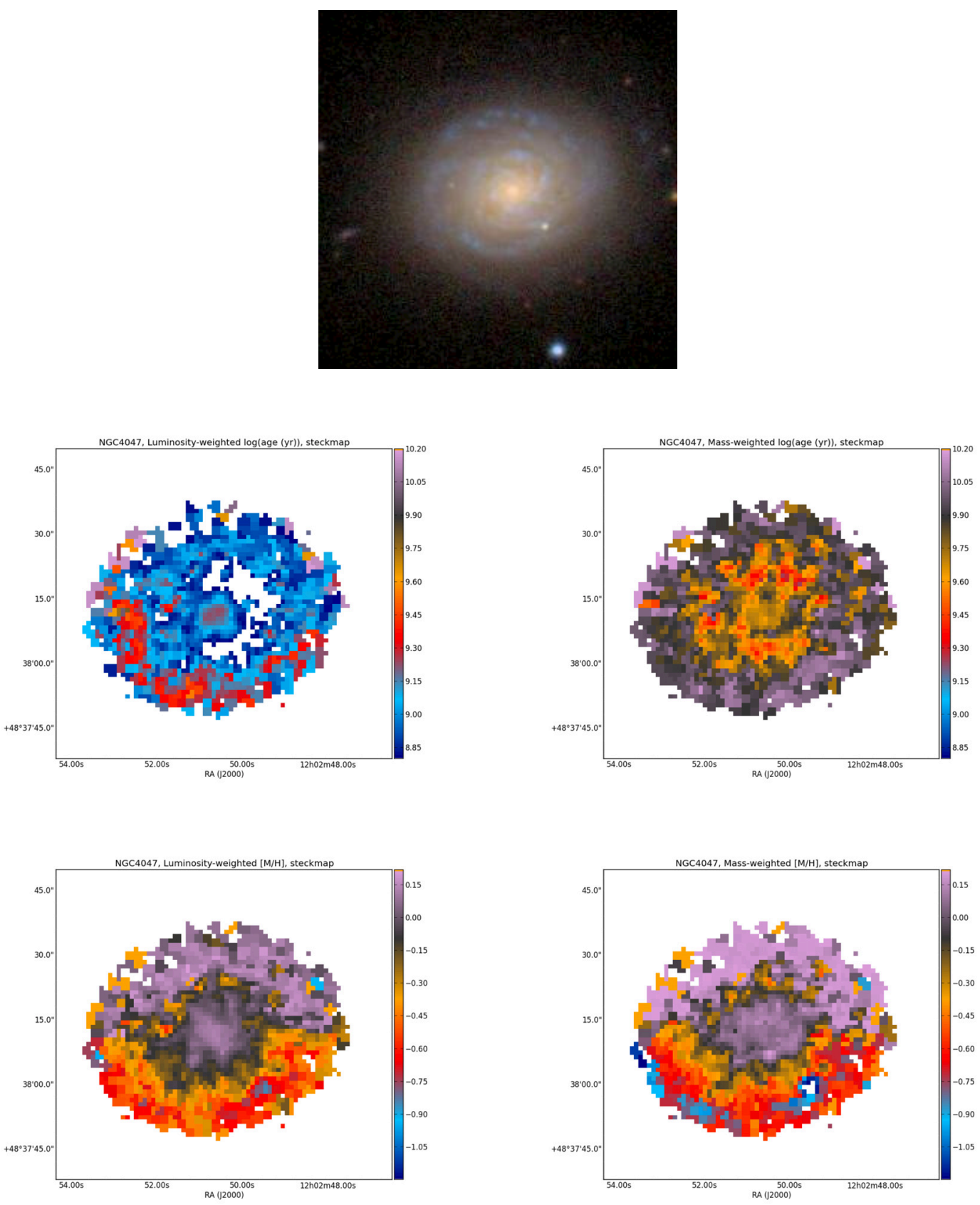

Fig. C.23. Mean log(age) and metallicity maps for NGC 4047. 
P. Sánchez-Blázquez et al.: Stellar populations in galaxy discs
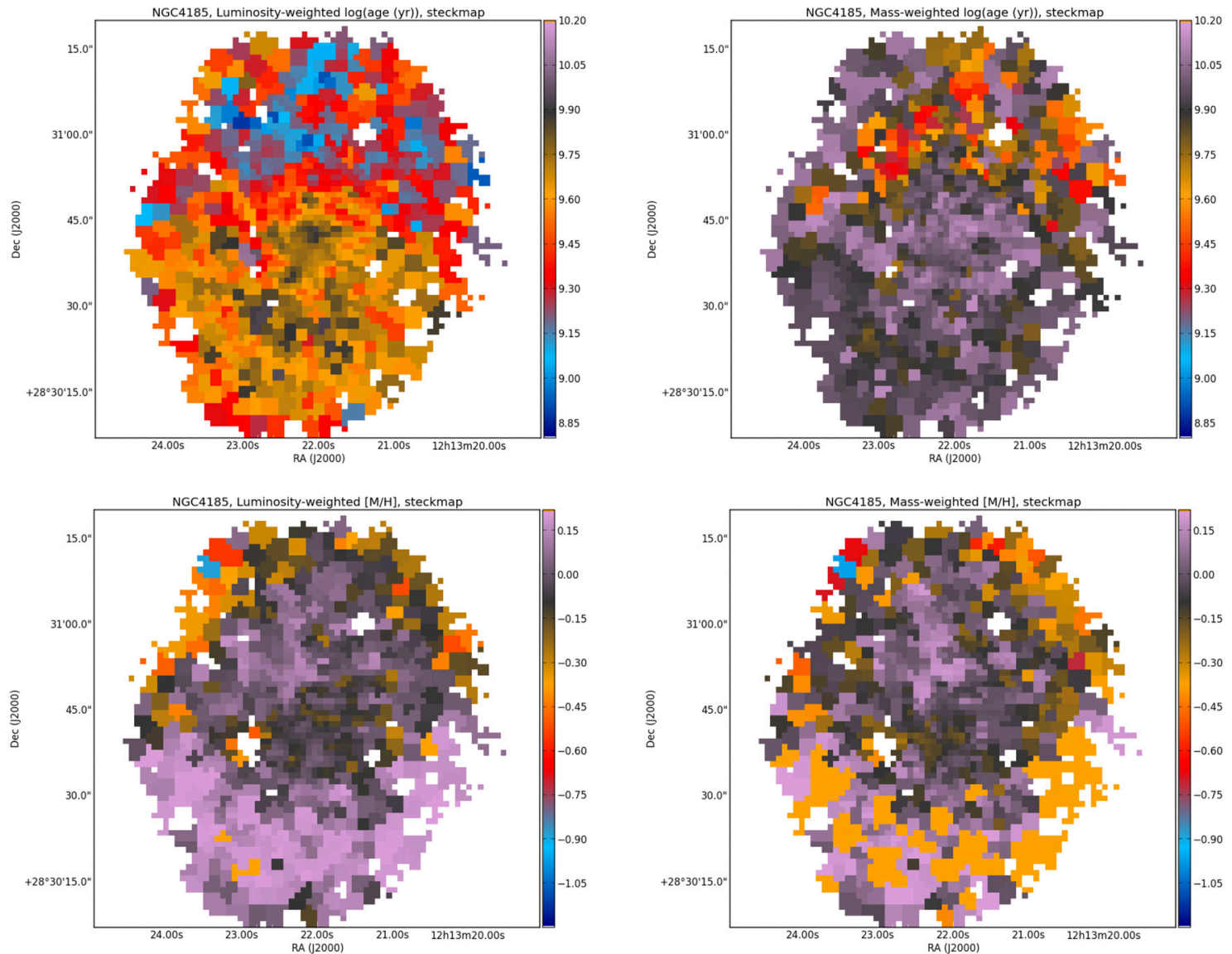

Fig. C.24. Mean log(age) and metallicity maps for NGC 4185 . 
A\&A 570, A6 (2014)
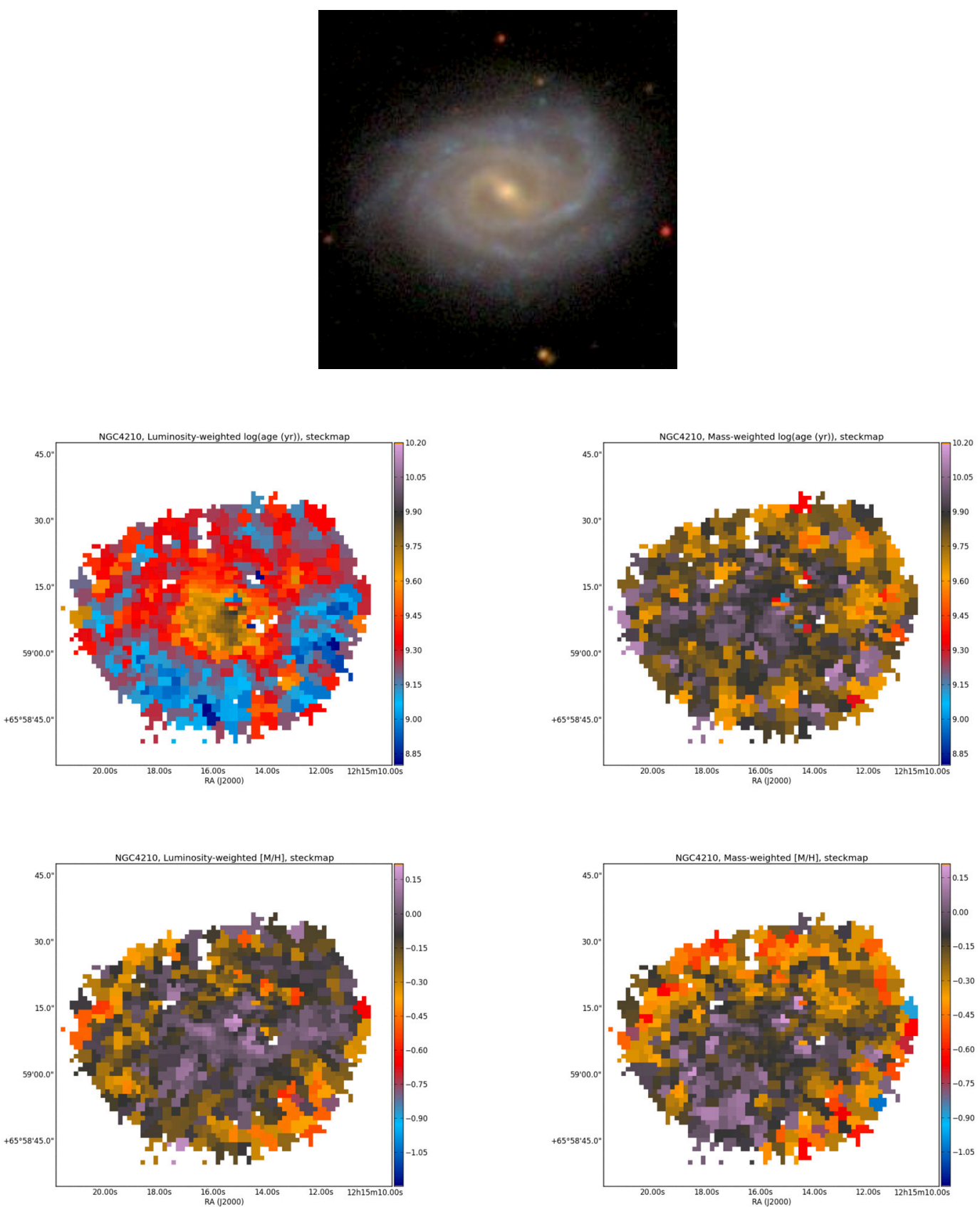

Fig. C.25. Mean log(age) and metallicity maps for NGC 4210. 
P. Sánchez-Blázquez et al.: Stellar populations in galaxy discs
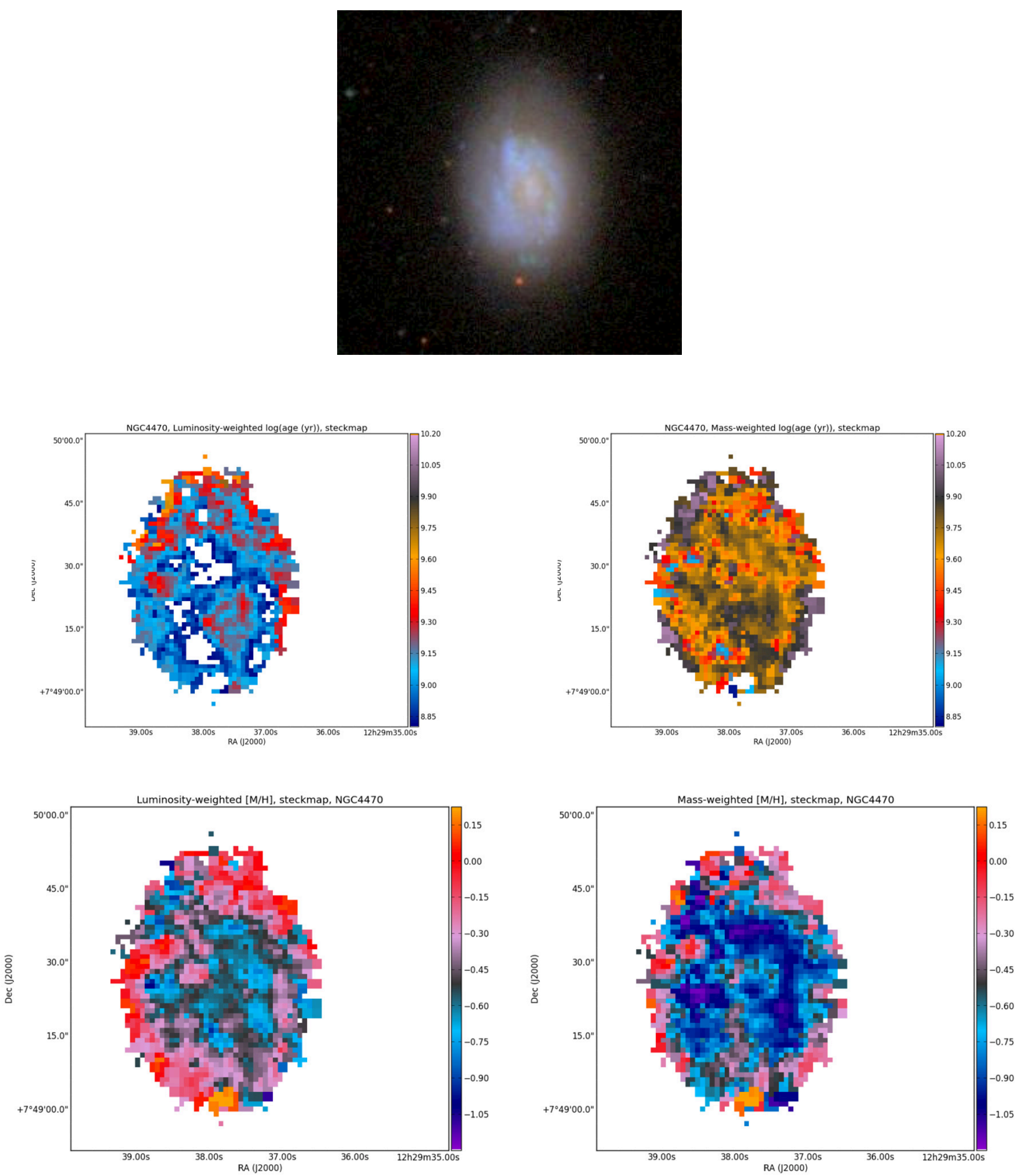

Fig. C.26. Mean log(age) and metallicity maps for NGC 4470 . 
A\&A 570, A6 (2014)
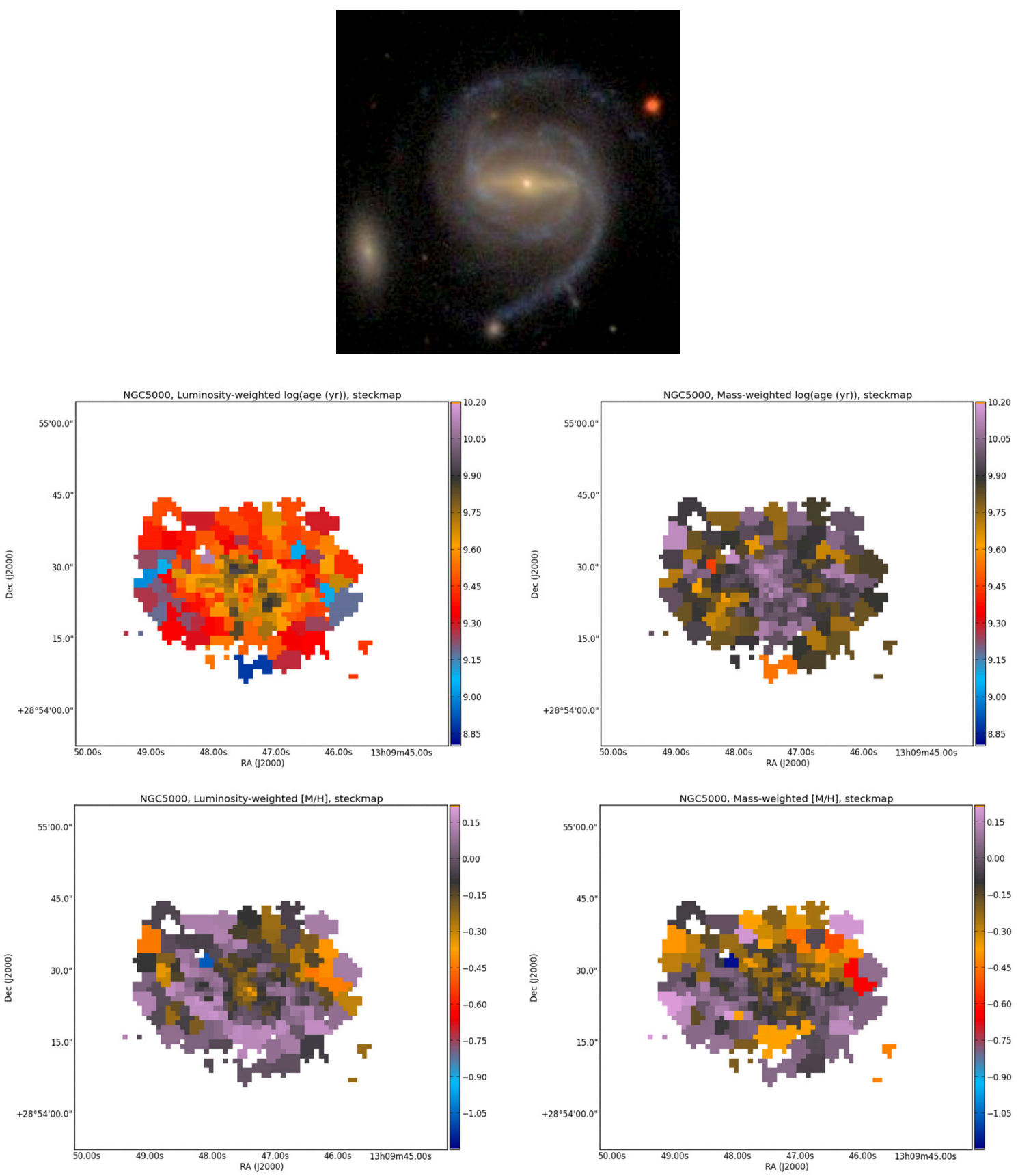

Fig. C.27. Mean log(age) and metallicity maps for NGC 5000. 
P. Sánchez-Blázquez et al.: Stellar populations in galaxy discs
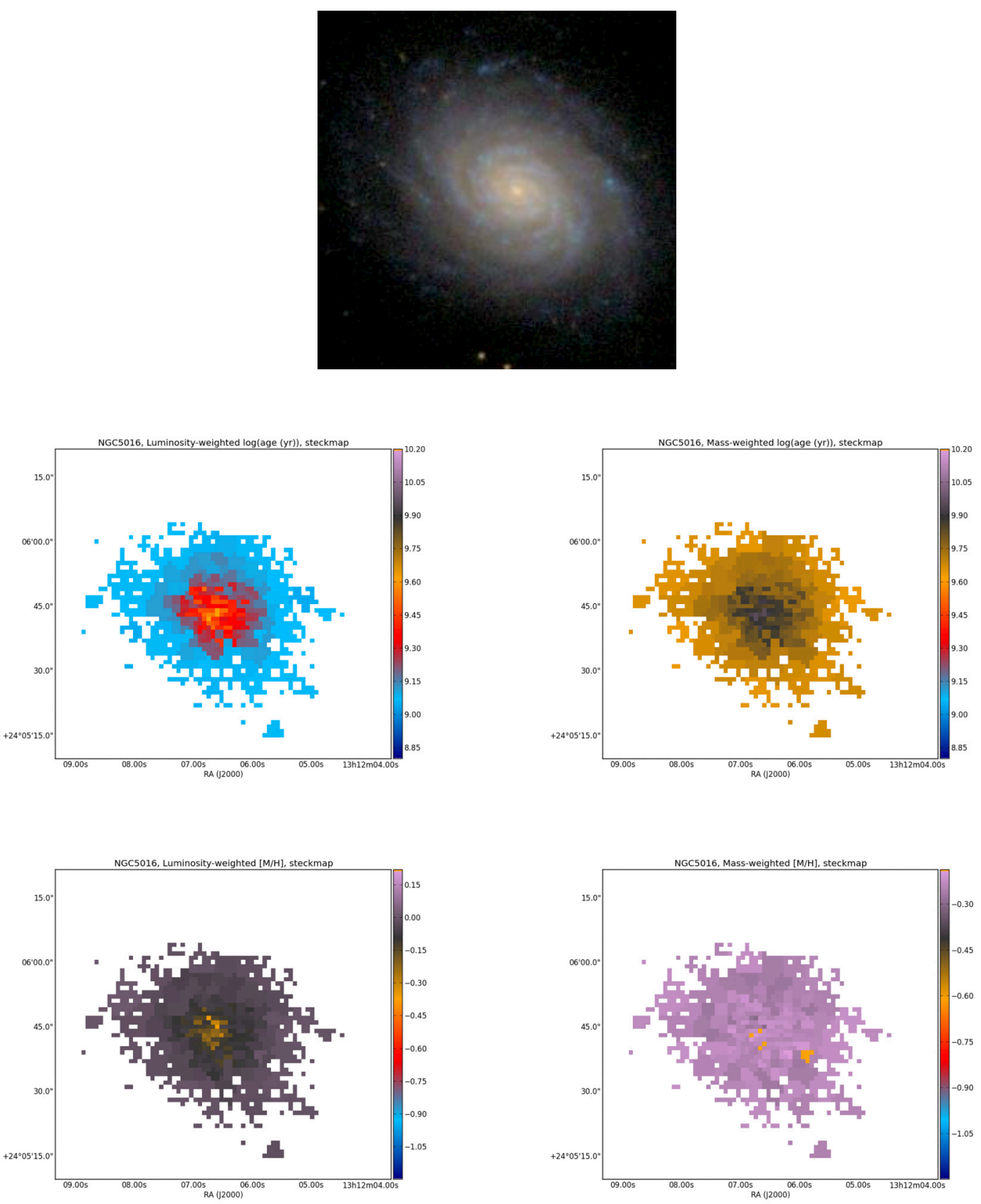

Fig. C.28. Mean log(age) and metallicity maps for NGC 5016 . 

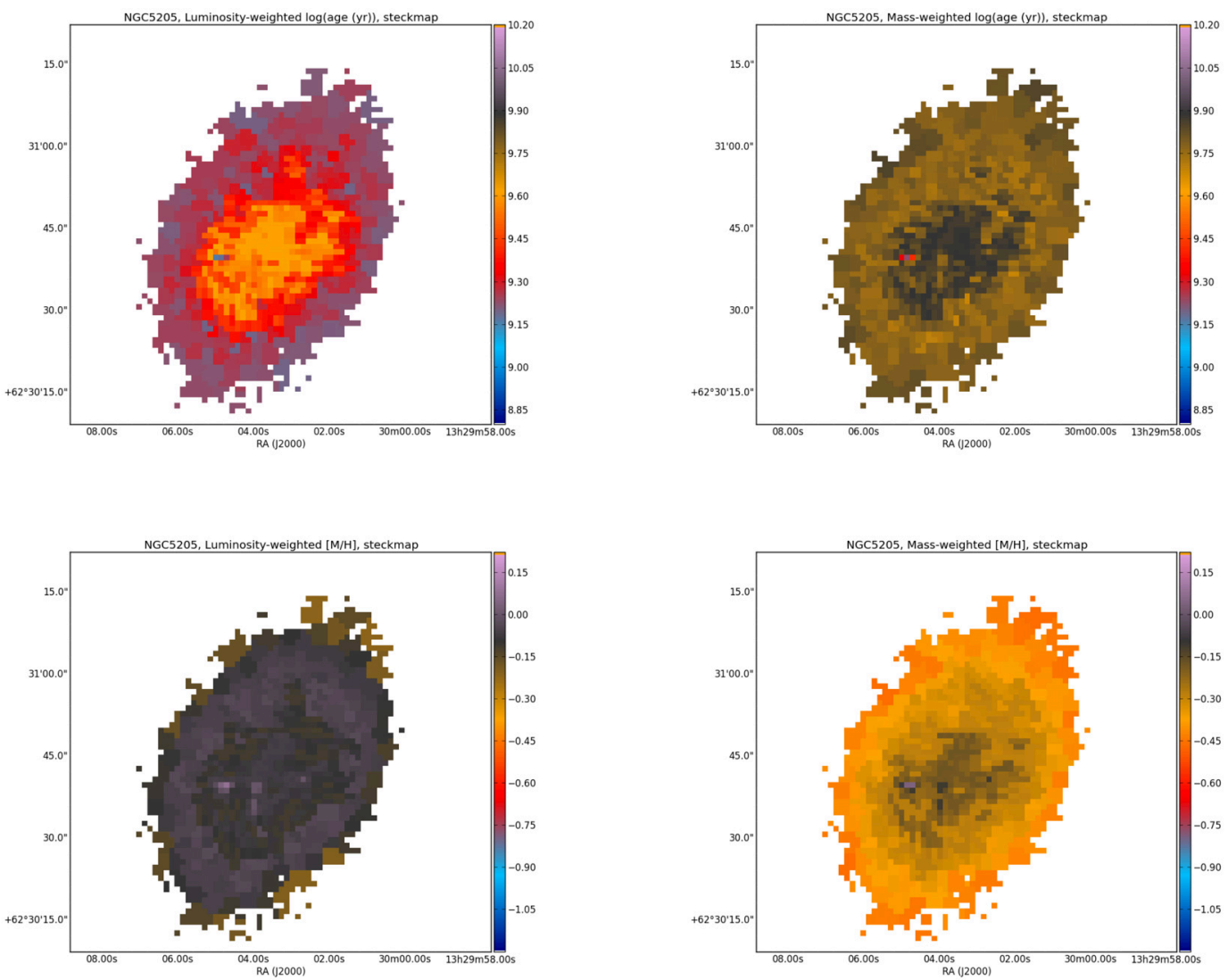

Fig. C.29. Mean log(age) and metallicity maps for NGC 5205. 
P. Sánchez-Blázquez et al.: Stellar populations in galaxy discs
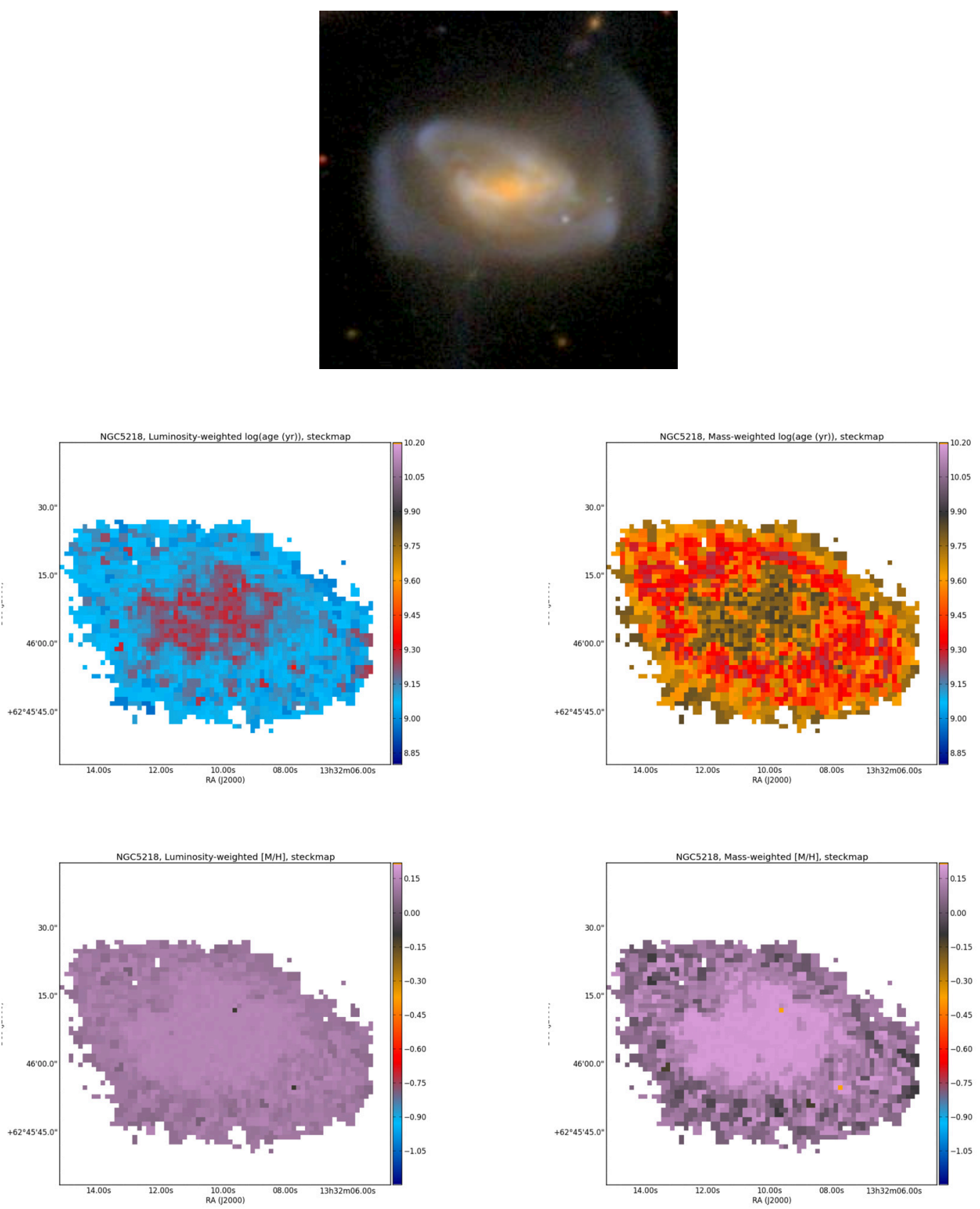

Fig. C.30. Mean log(age) and metallicity maps for NGC 5218. 

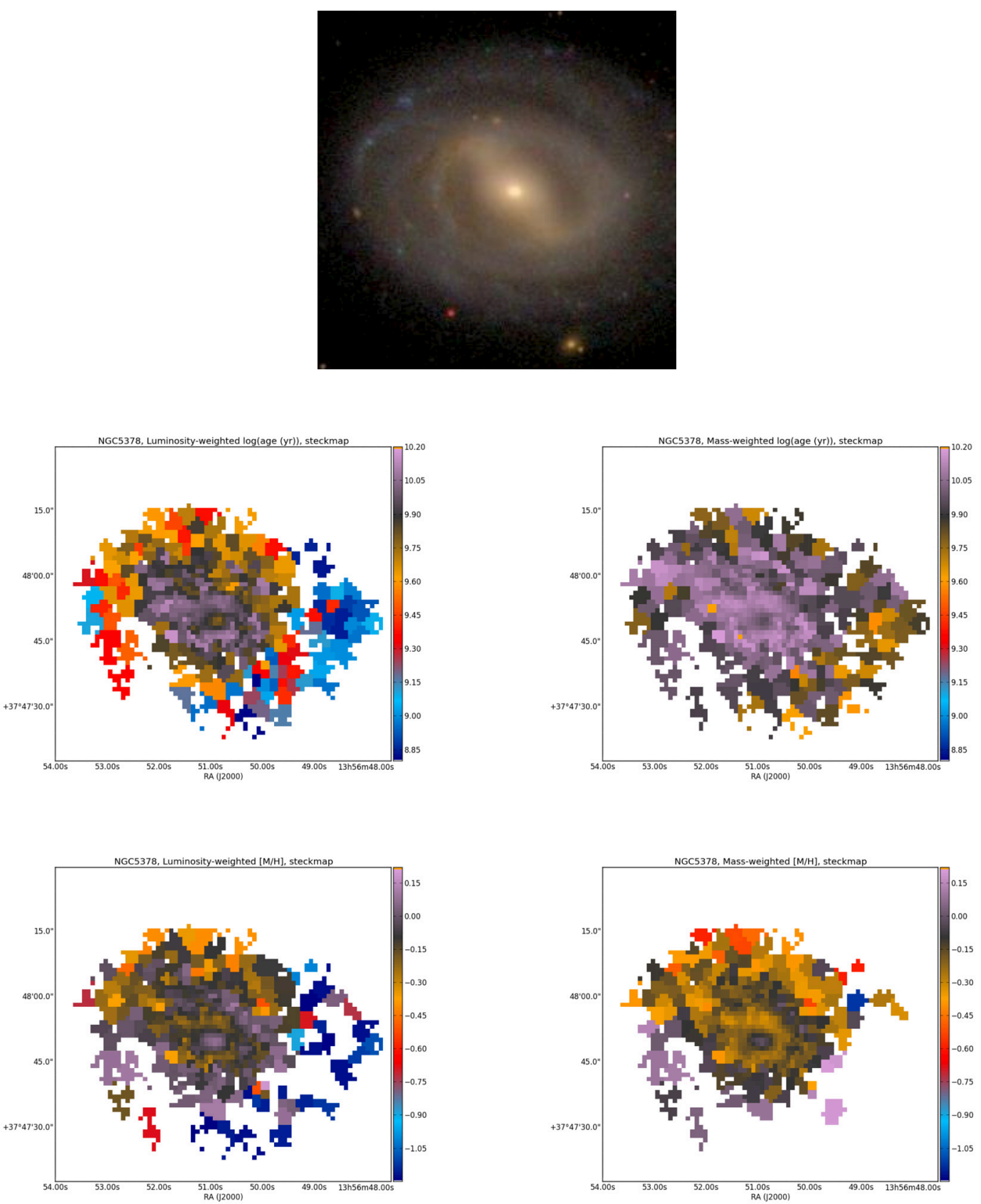

Fig. C.31. Mean log(age) and metallicity maps for NGC 5378. 
P. Sánchez-Blázquez et al.: Stellar populations in galaxy discs
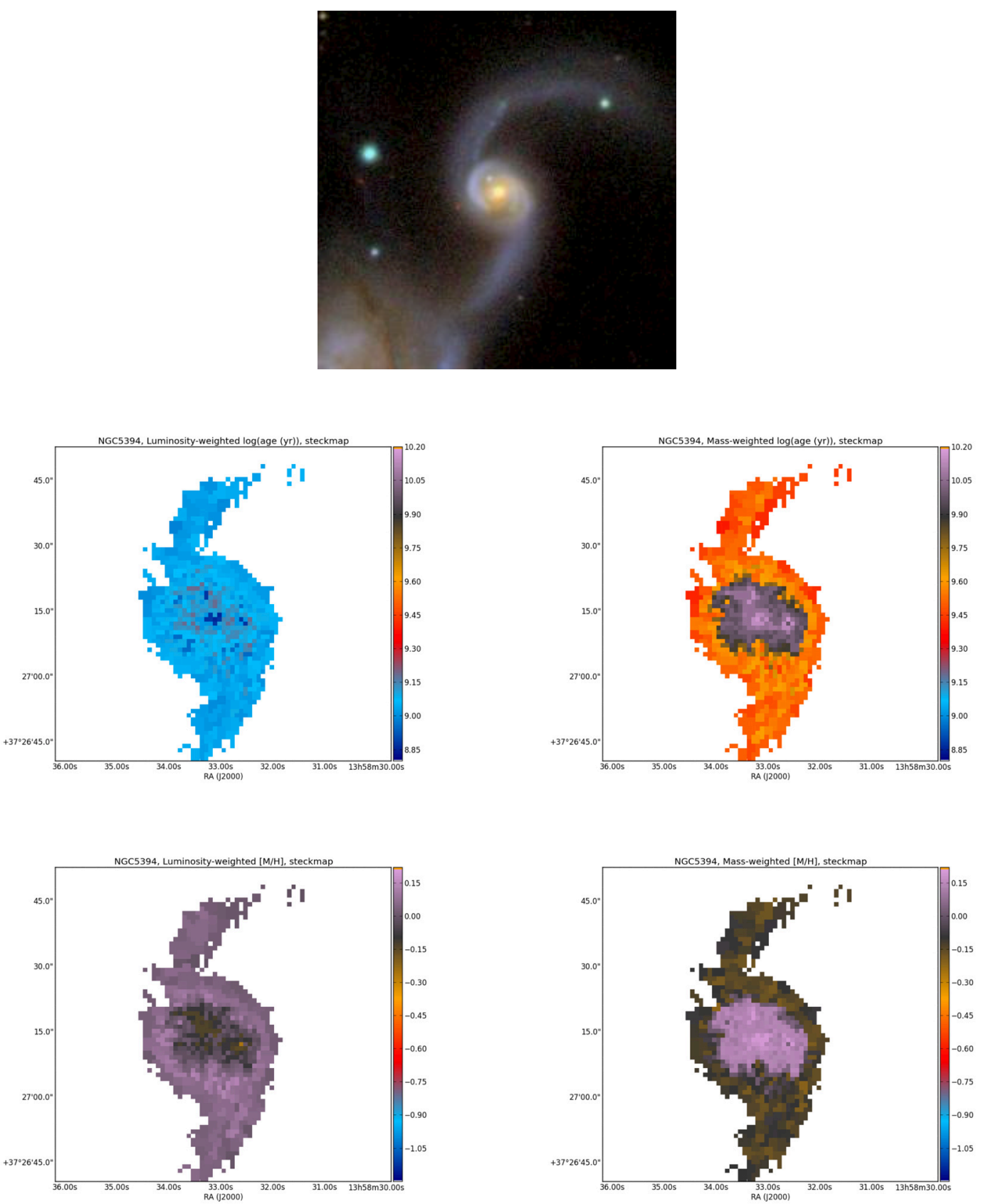

Fig. C.32. Mean log(age) and metallicity maps for NGC 5394. 

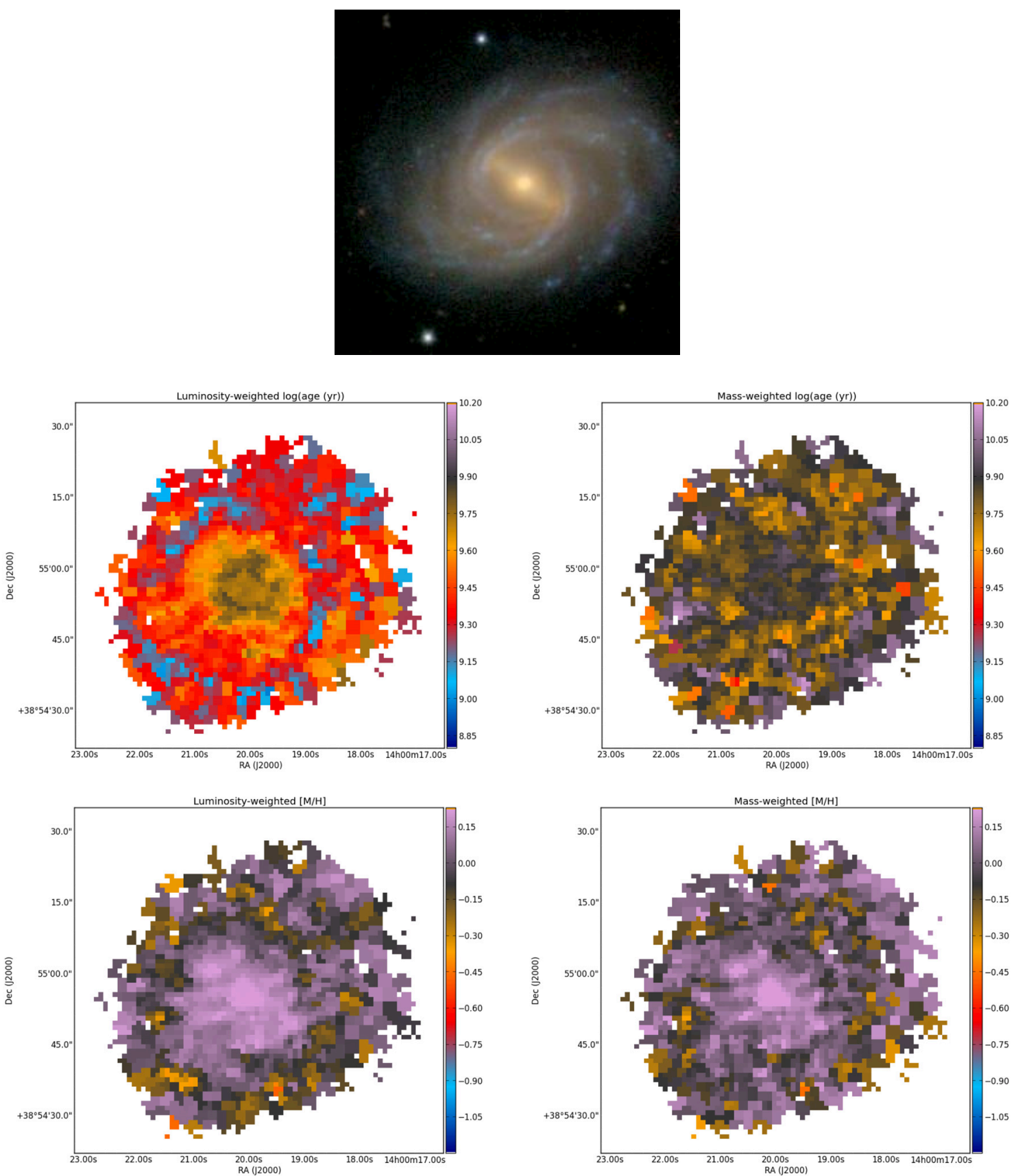

Fig. C.33. Mean log(age) and metallicity maps for NGC 5406. 
P. Sánchez-Blázquez et al.: Stellar populations in galaxy discs
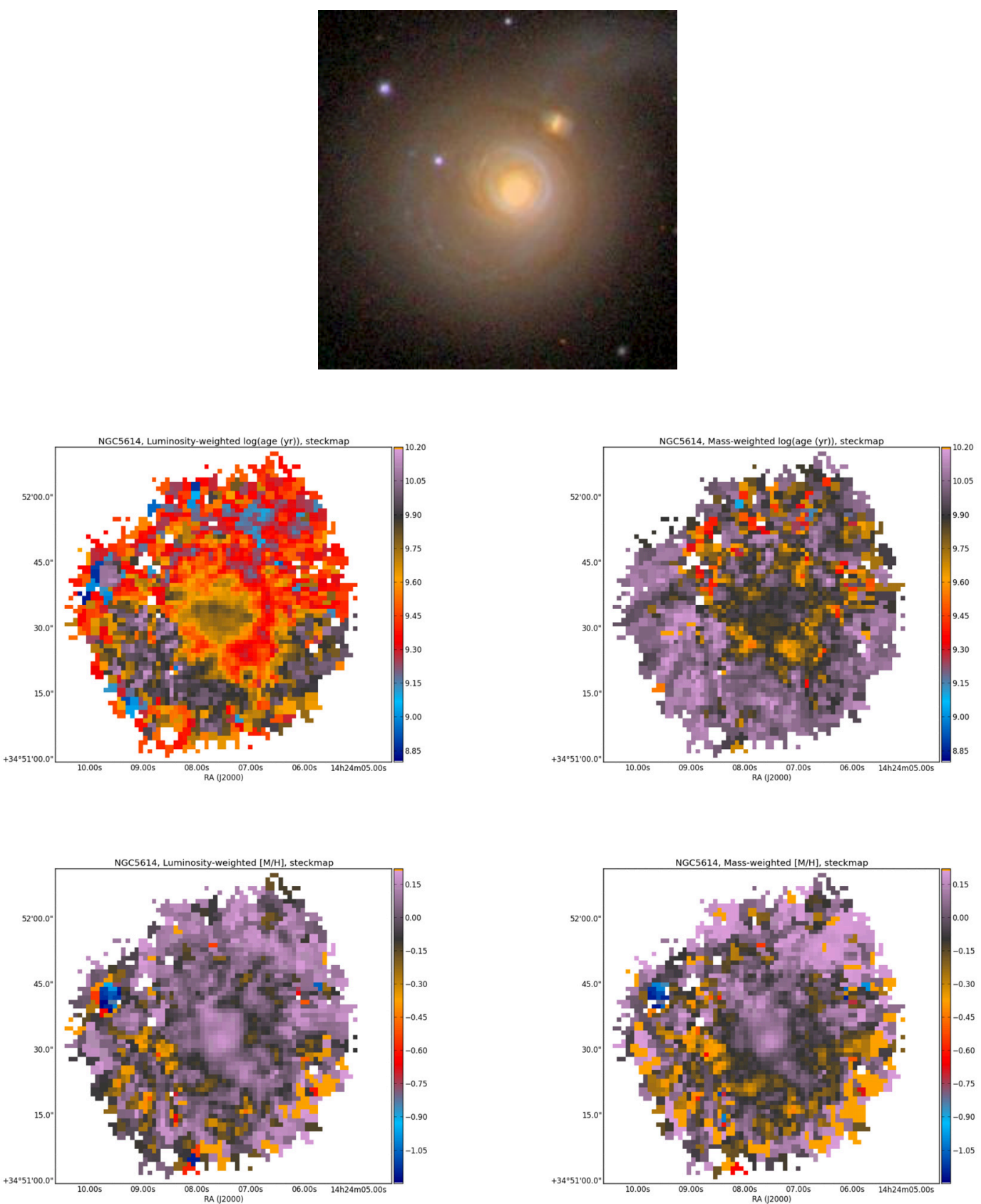

Fig. C.34. Mean log(age) and metallicity maps for NGC 5614. 
A\&A 570, A6 (2014)
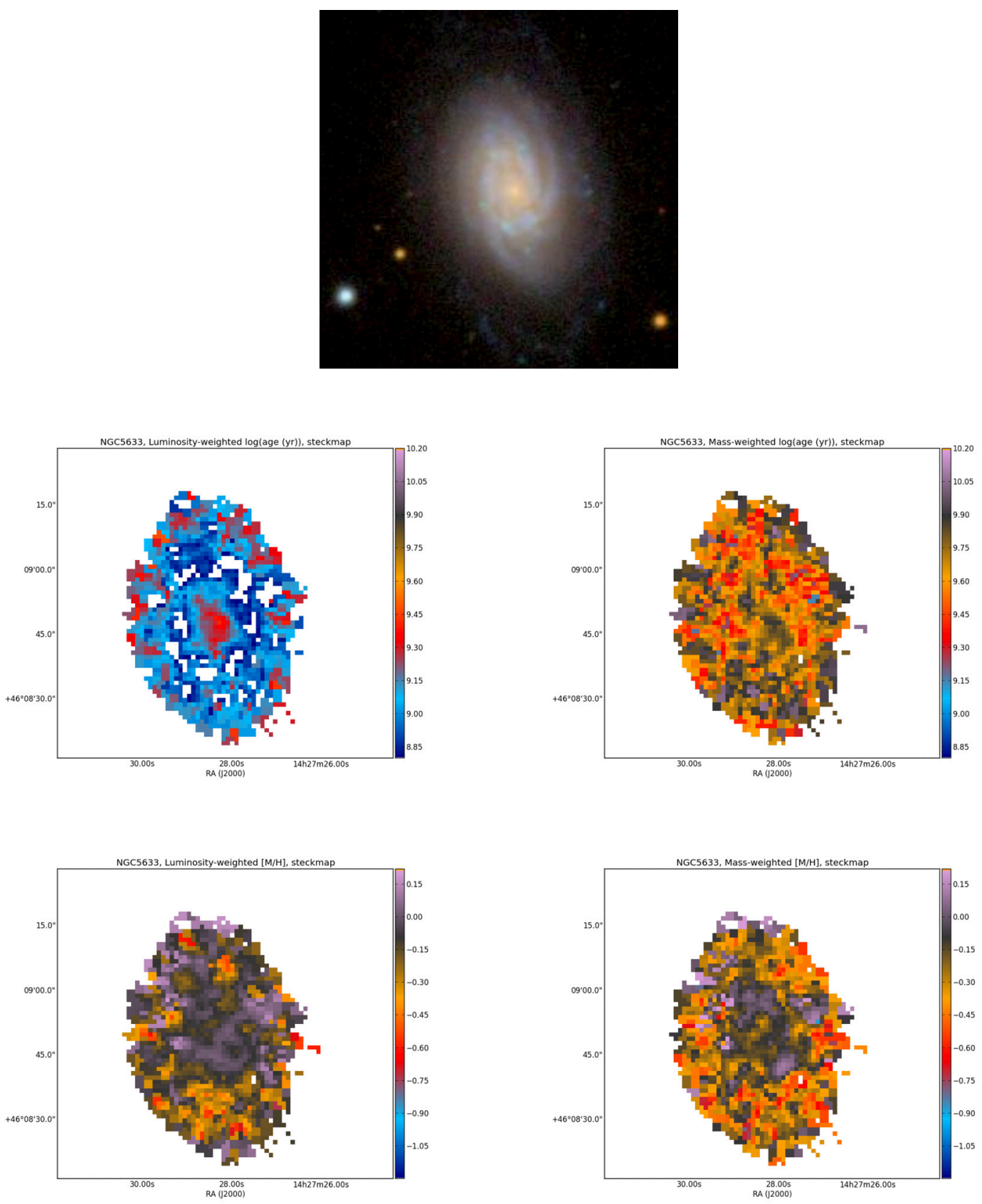

Fig. C.35. Mean log(age) and metallicity maps for NGC 5633. 
P. Sánchez-Blázquez et al.: Stellar populations in galaxy discs
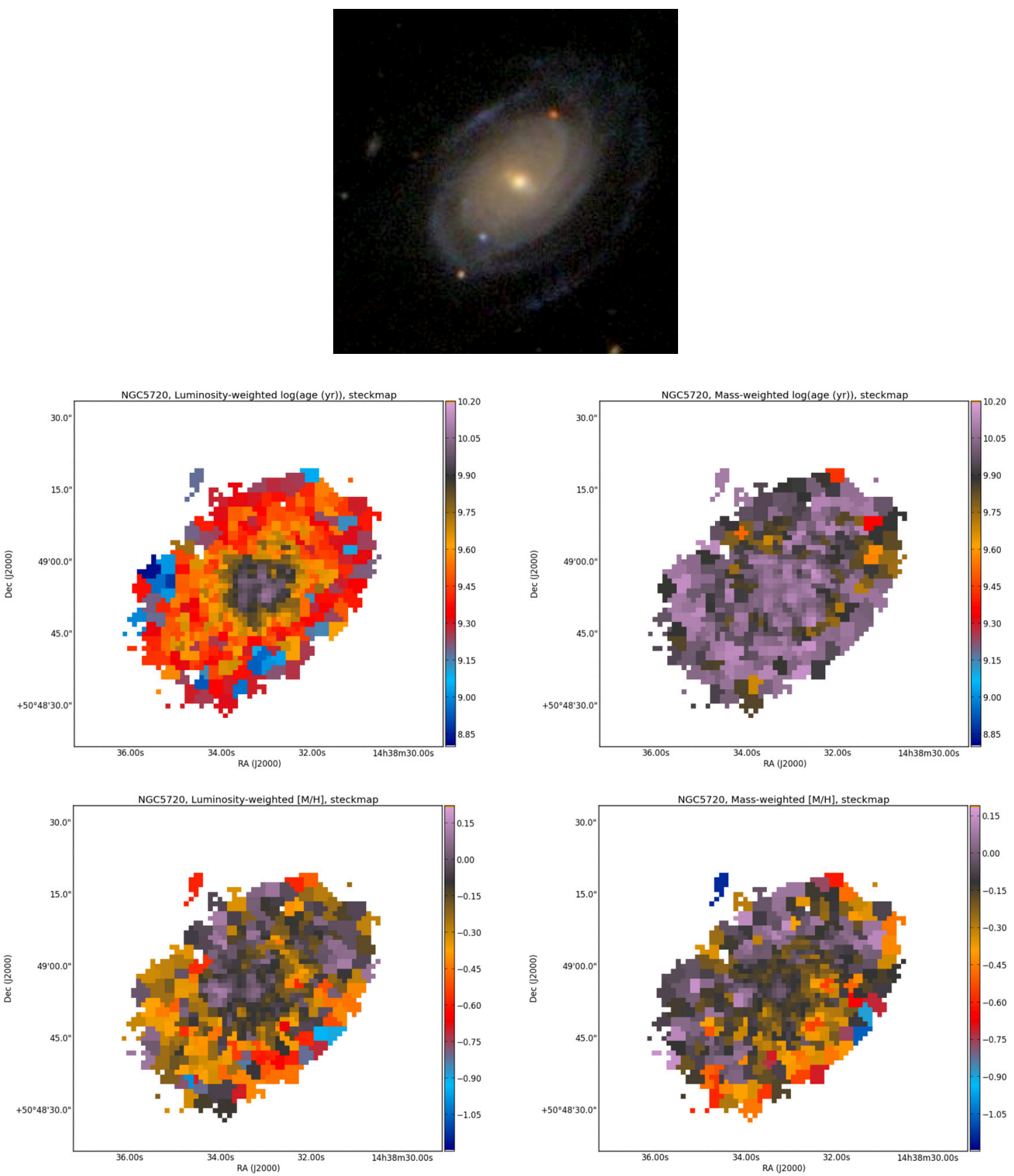

Fig. C.36. Mean log(age) and metallicity maps for NGC 5720. 
A\&A 570, A6 (2014)
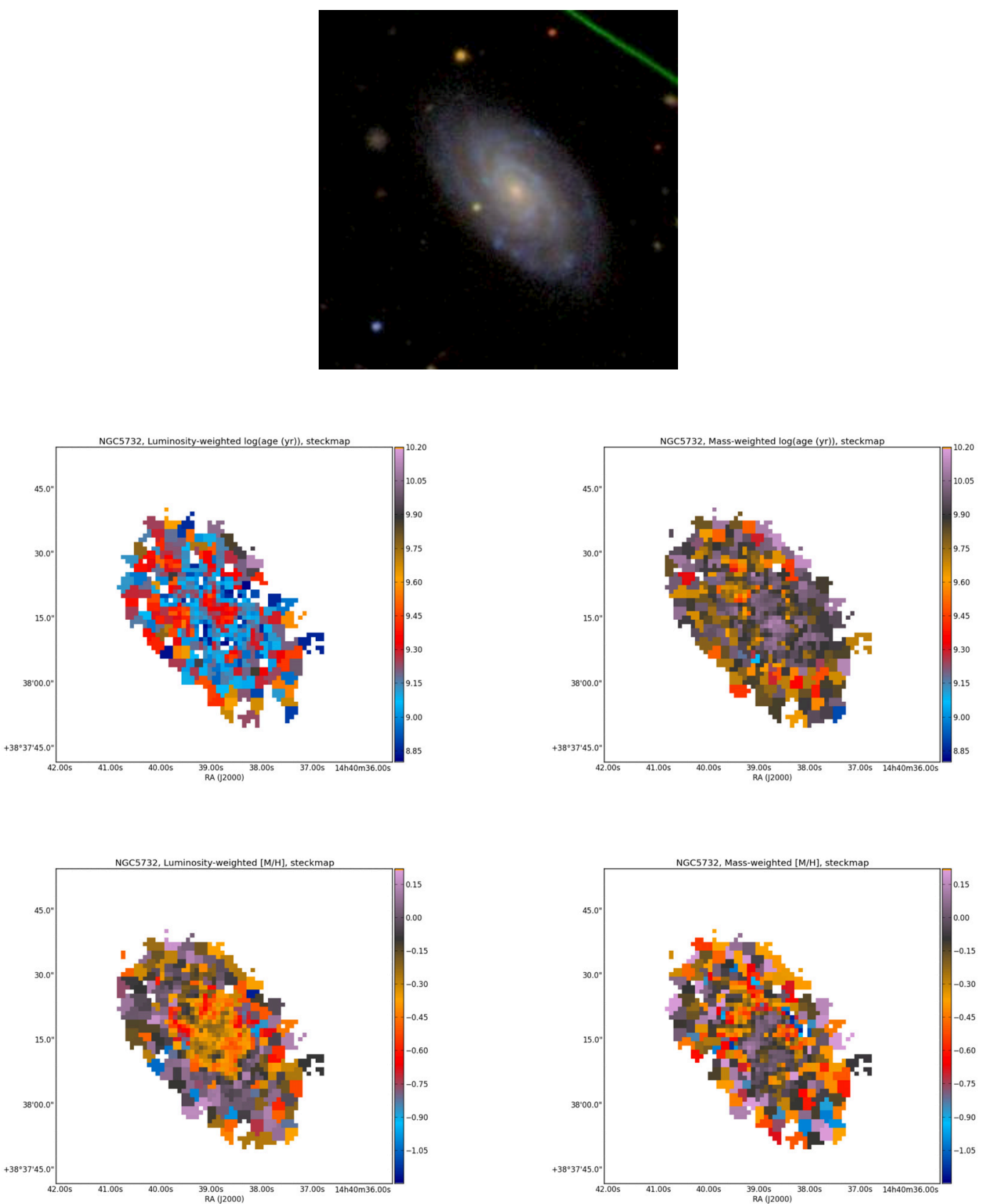

Fig. C.37. Mean log(age) and metallicity maps for NGC 5732. 
P. Sánchez-Blázquez et al.: Stellar populations in galaxy discs
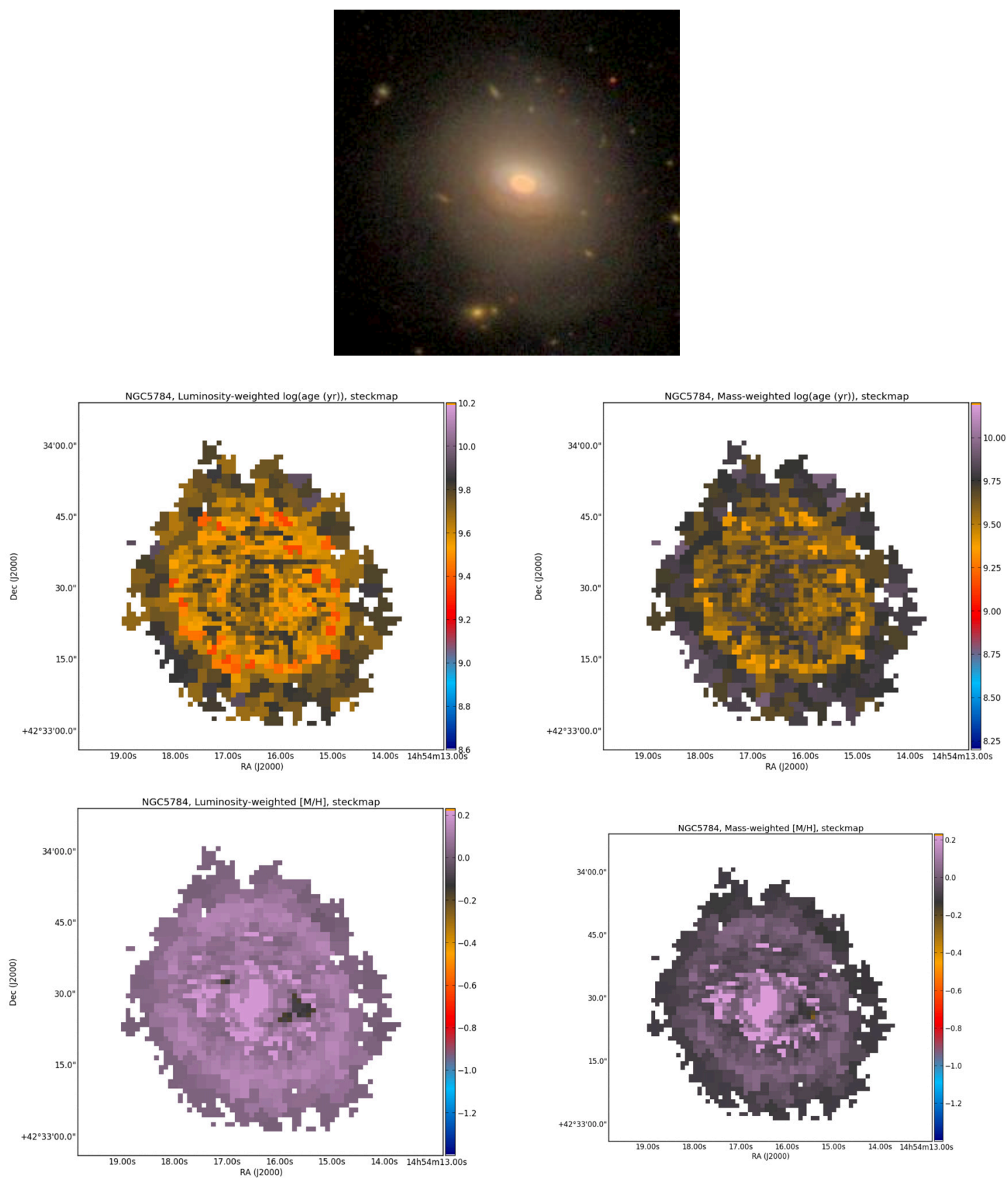

Fig. C.38. Mean log(age) and metallicity maps for NGC 5784 . 

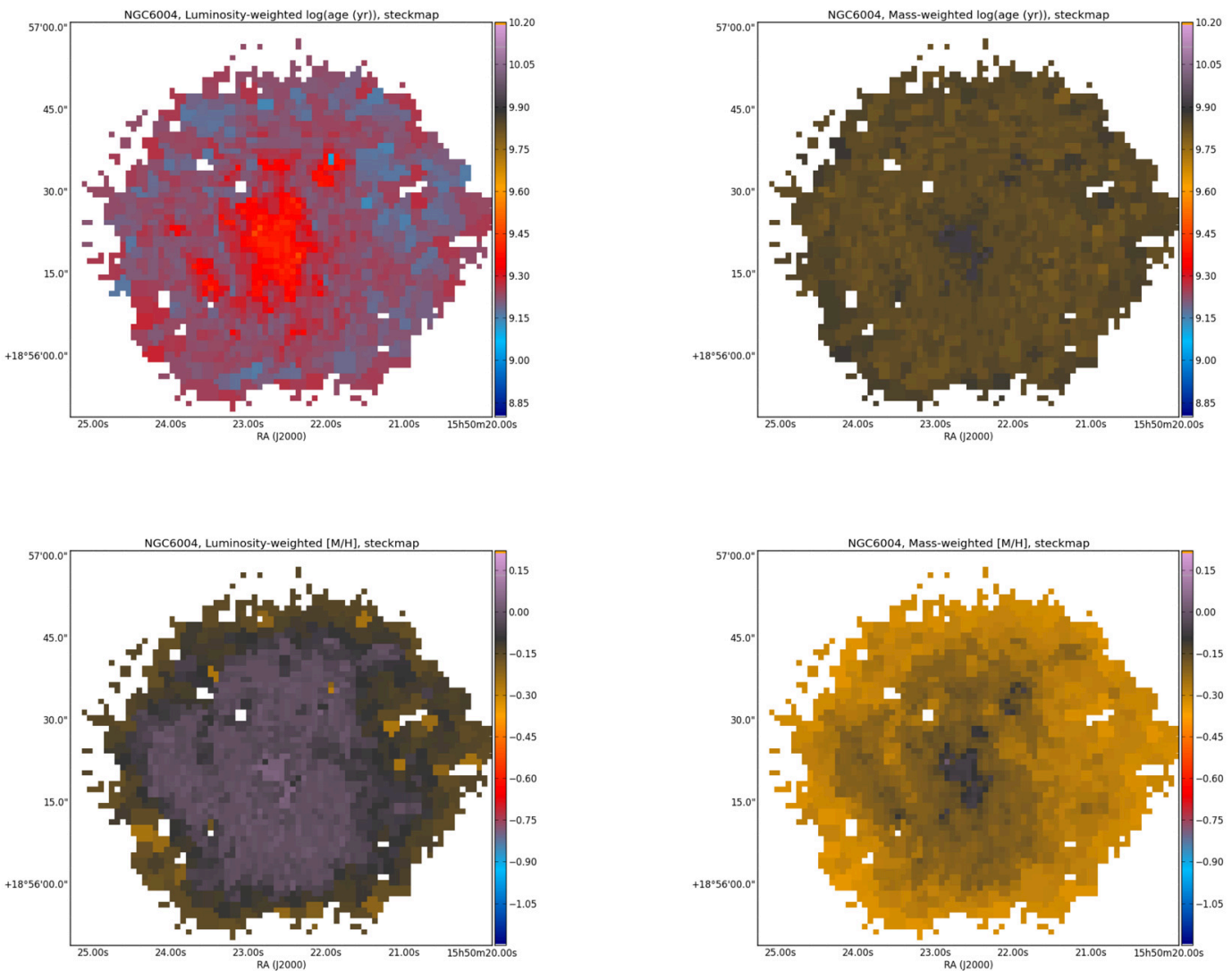

Fig. C.39. Mean log(age) and metallicity maps for NGC 6004. 
P. Sánchez-Blázquez et al.: Stellar populations in galaxy discs
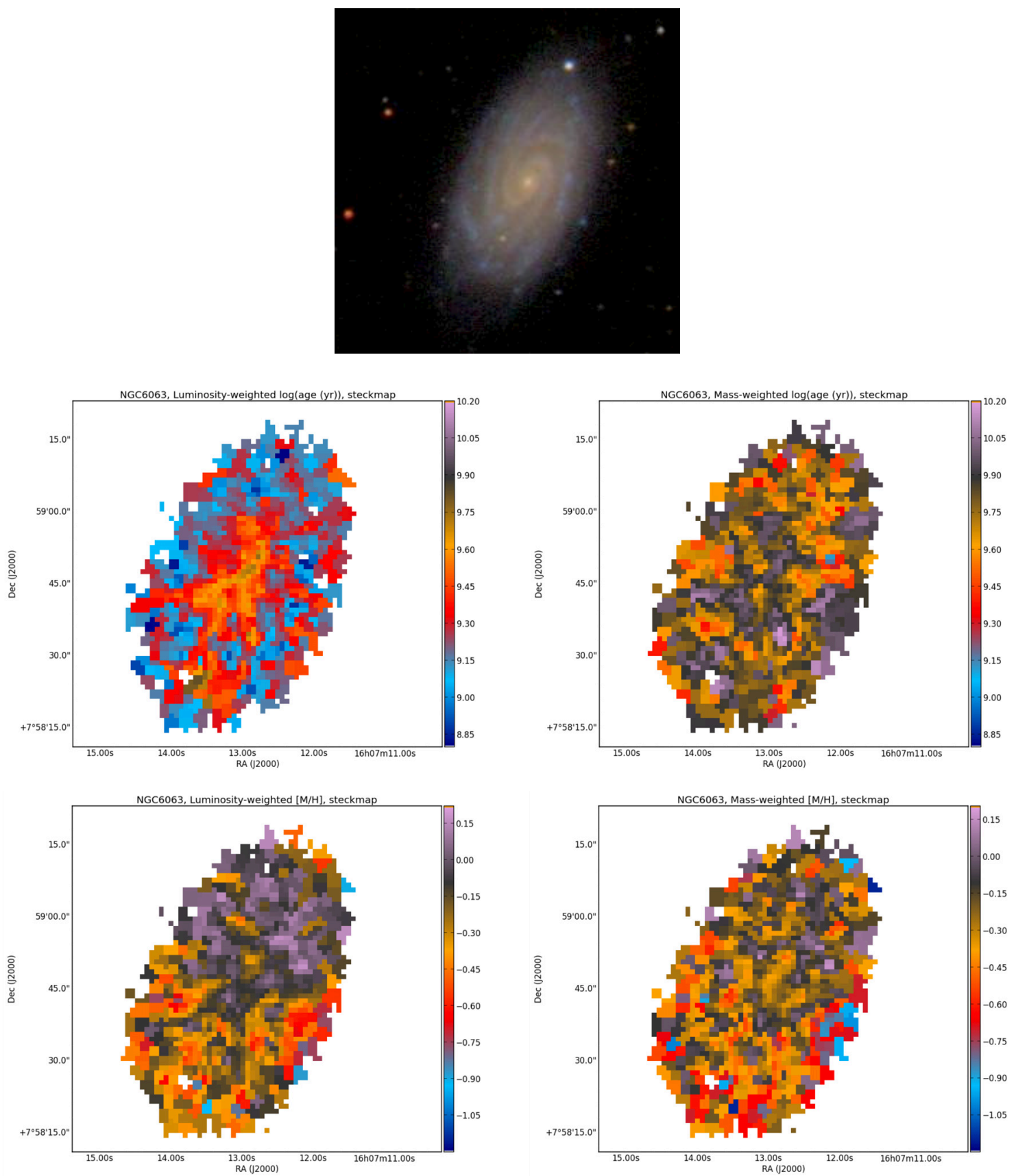

Fig. C.40. Mean log(age) and metallicity maps for NGC 6063. 

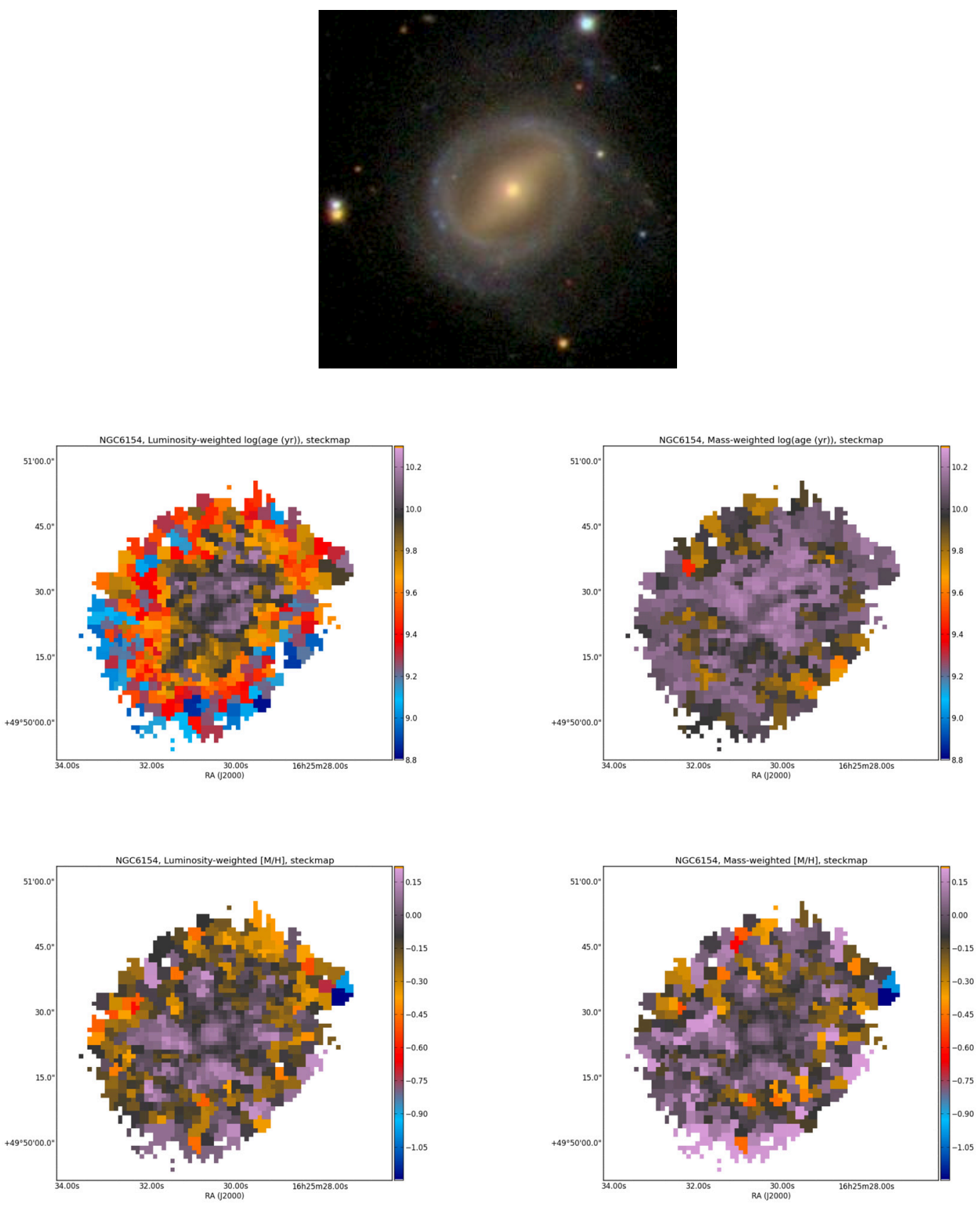

Fig. C.41. Mean log(age) and metallicity maps for NGC 6154. 
P. Sánchez-Blázquez et al.: Stellar populations in galaxy discs
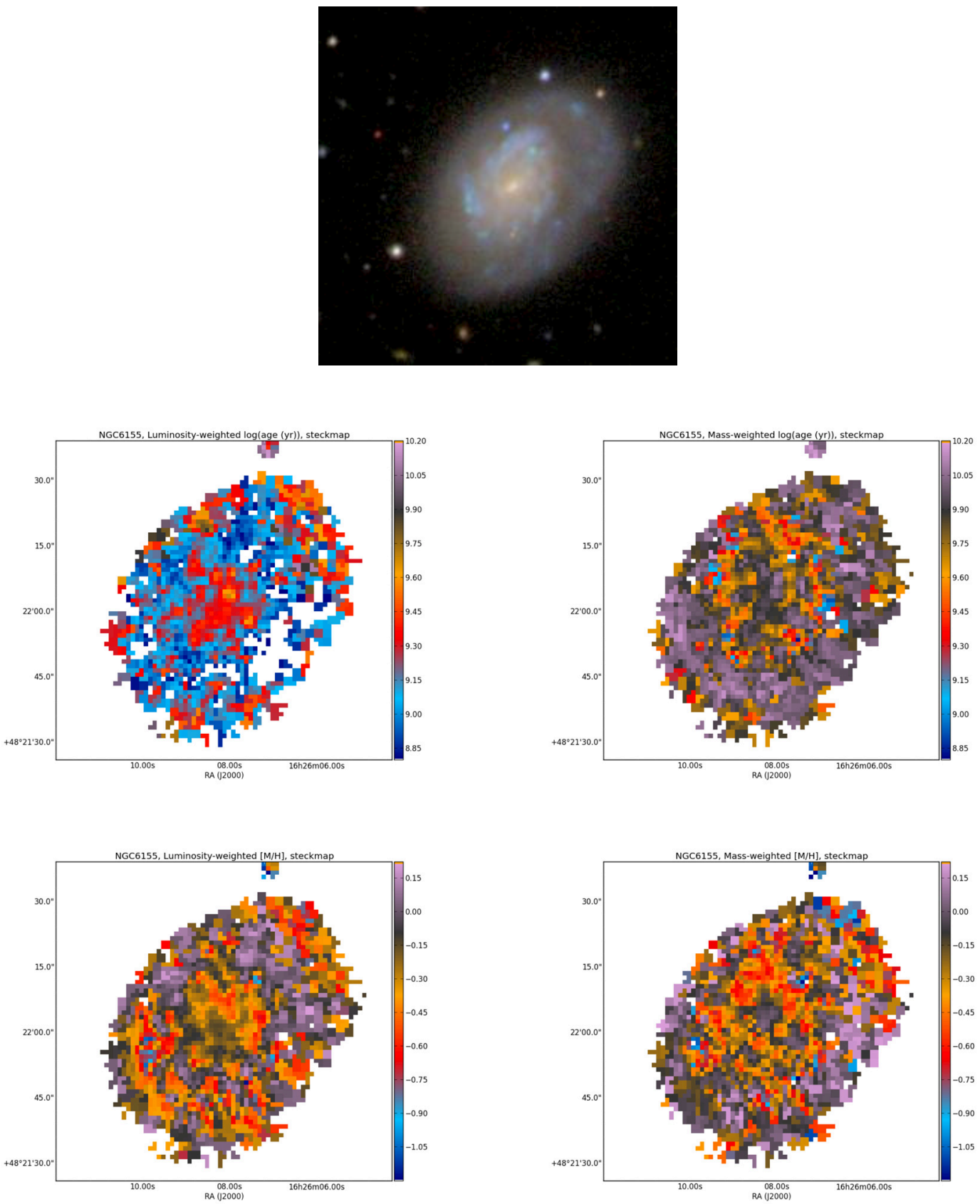

Fig. C.42. Mean log(age) and metallicity maps for NGC 6155 . 
A\&A 570, A6 (2014)
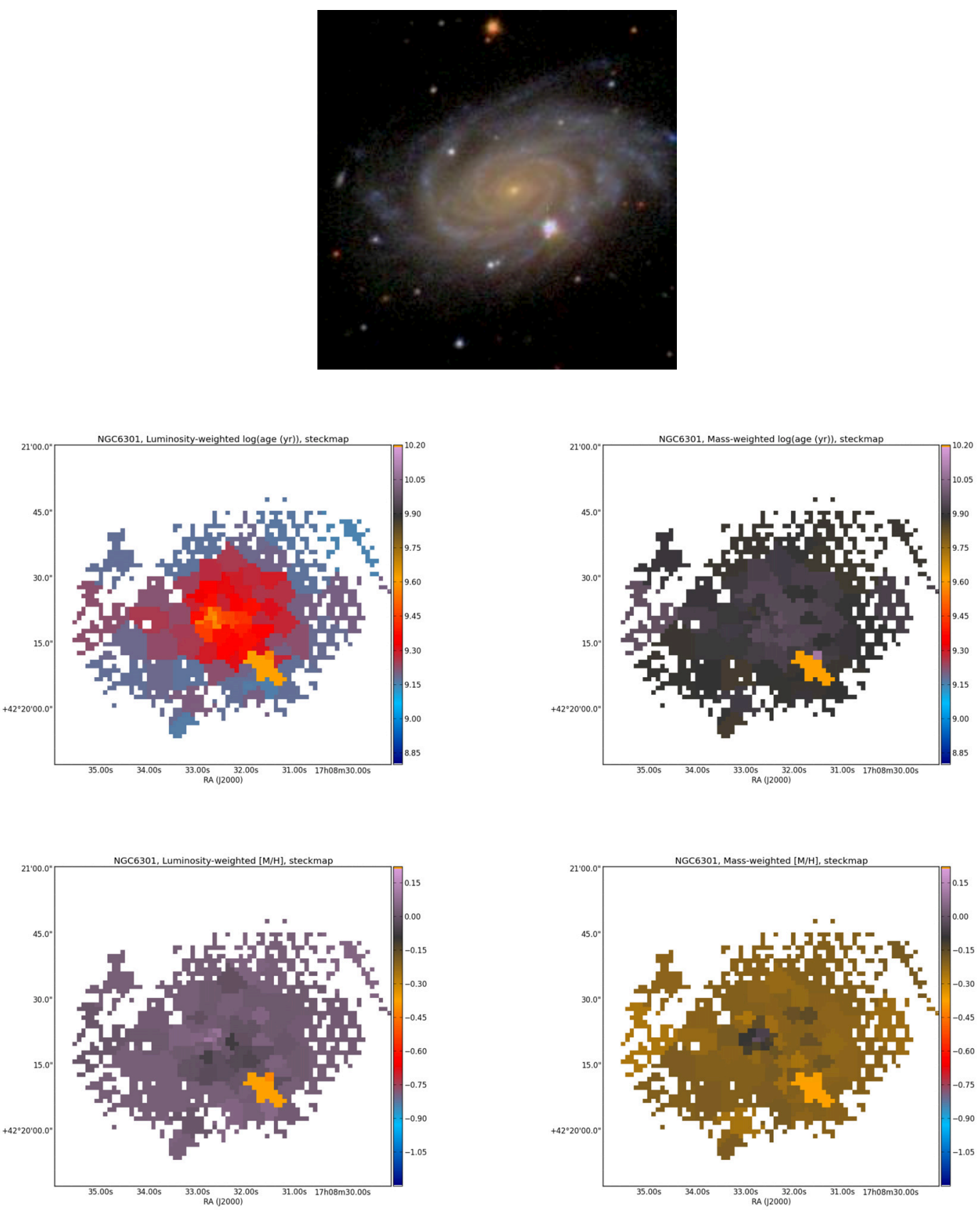

Fig. C.43. Mean log(age) and metallicity maps for NGC 6301. 
P. Sánchez-Blázquez et al.: Stellar populations in galaxy discs
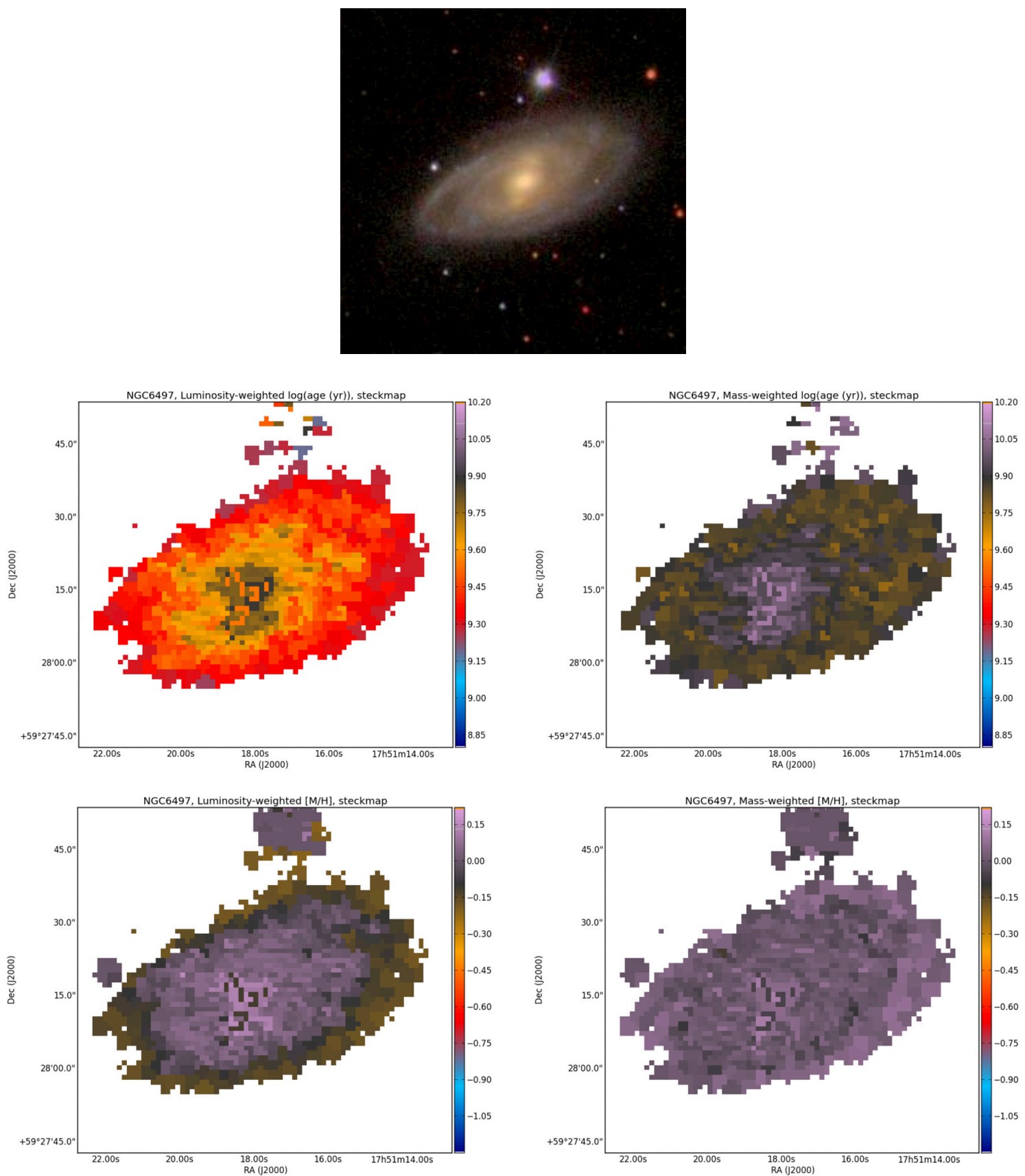

Fig. C.44. Mean log(age) and metallicity maps for NGC 6497. 
A\&A 570, A6 (2014)
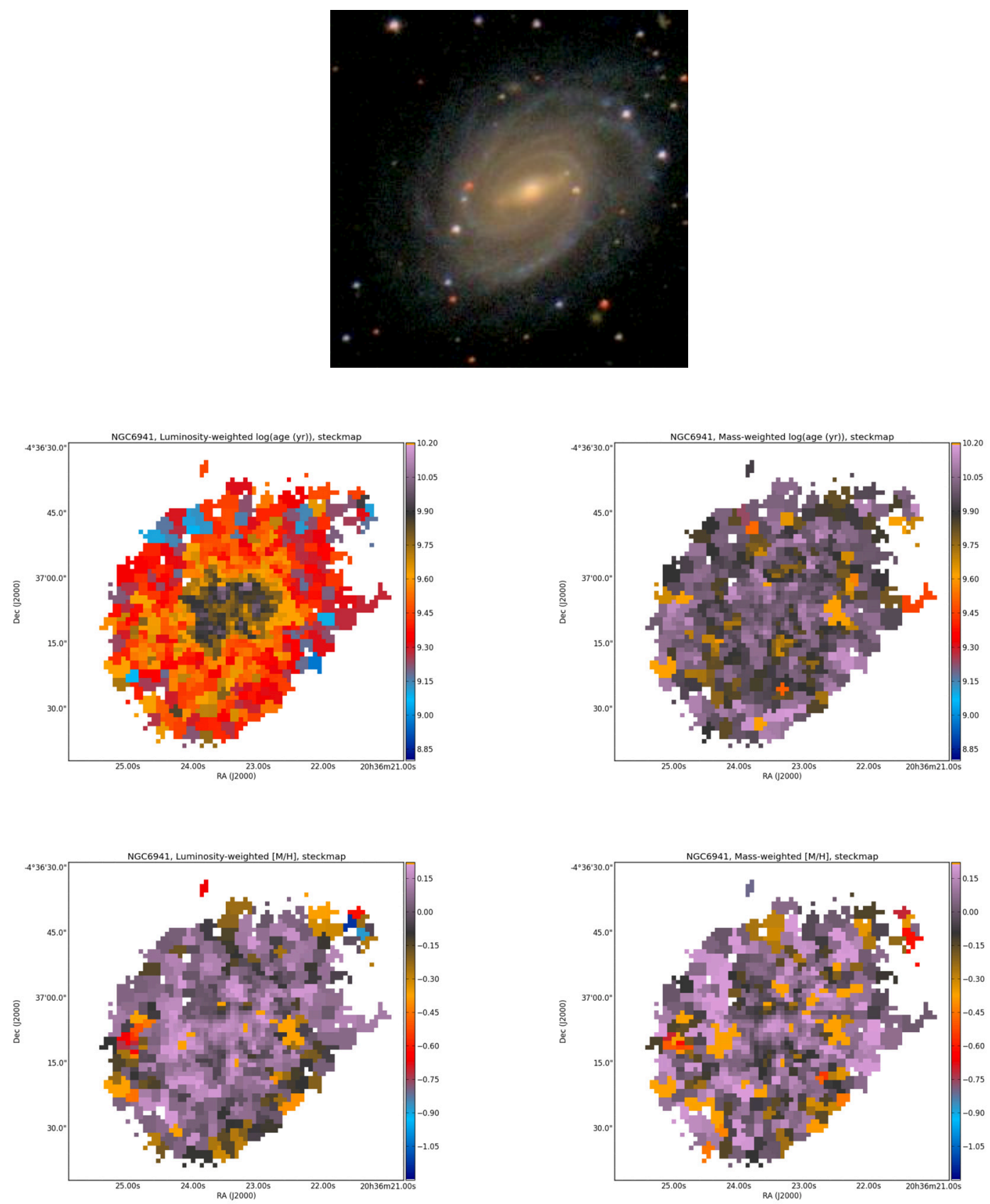

Fig. C.45. Mean log(age) and metallicity maps for NGC 6941. 
P. Sánchez-Blázquez et al.: Stellar populations in galaxy discs
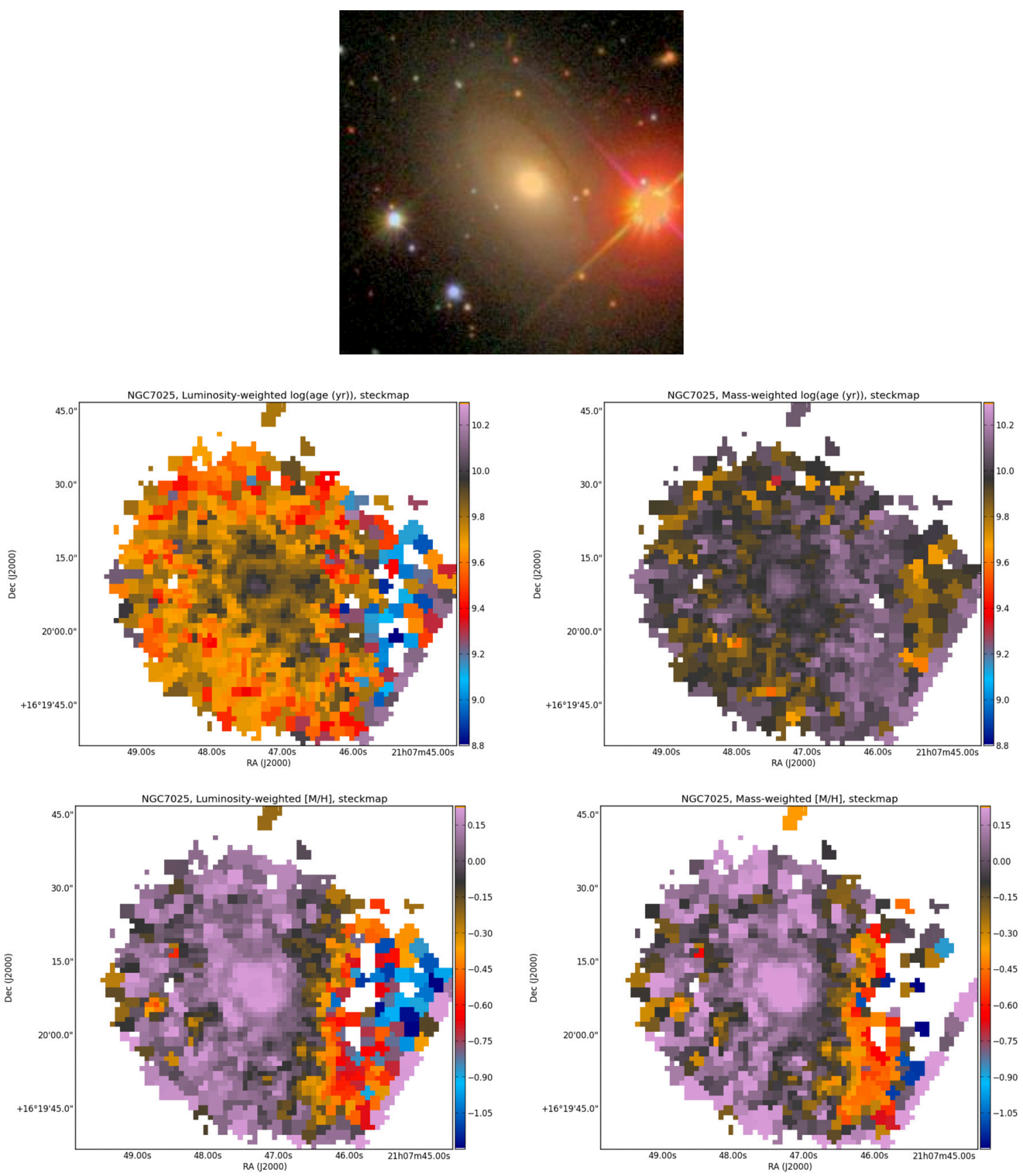

Fig. C.46. Mean log(age) and metallicity maps for NGC 7025. 
A\&A 570, A6 (2014)
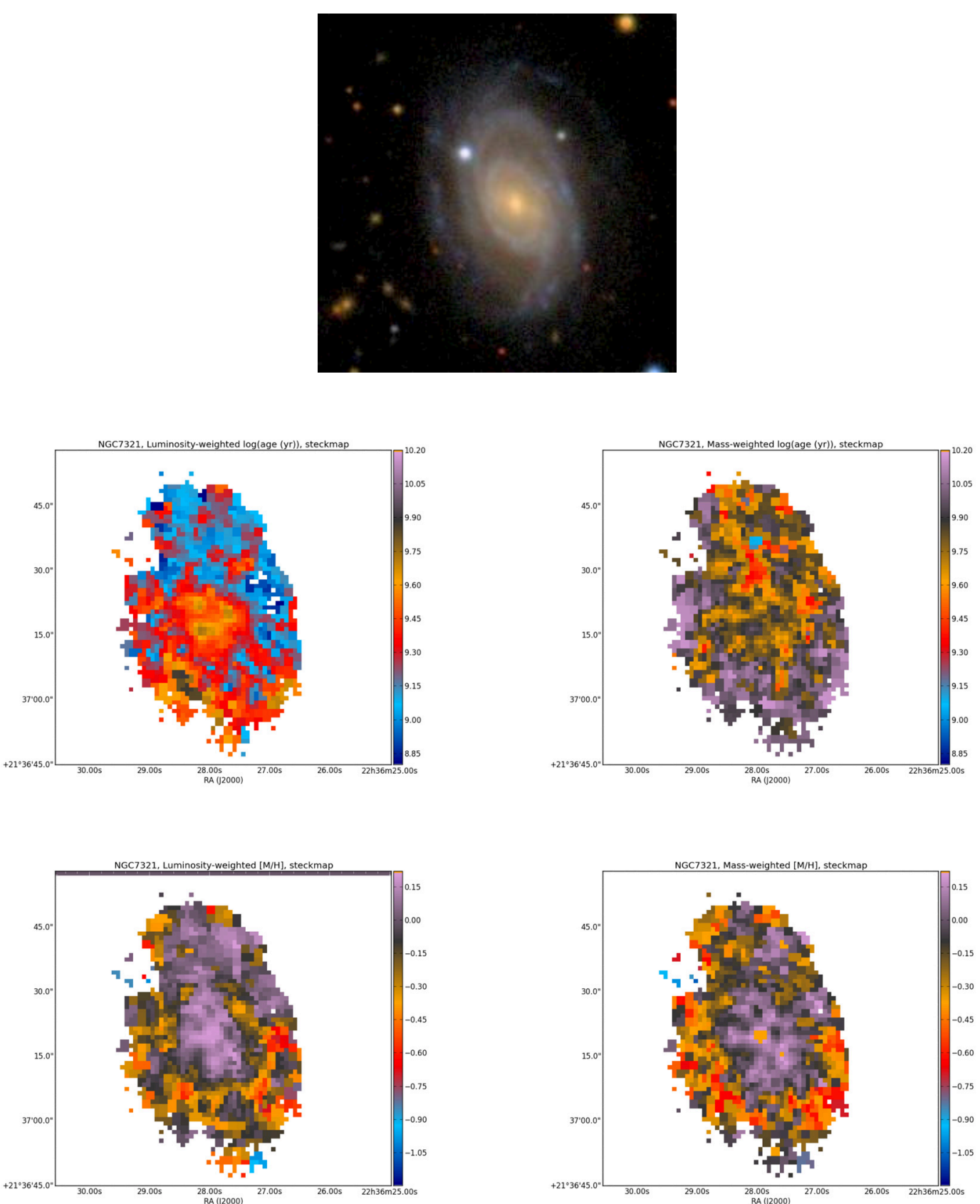

Fig. C.47. Mean log(age) and metallicity maps for NGC 7321. 
P. Sánchez-Blázquez et al.: Stellar populations in galaxy discs
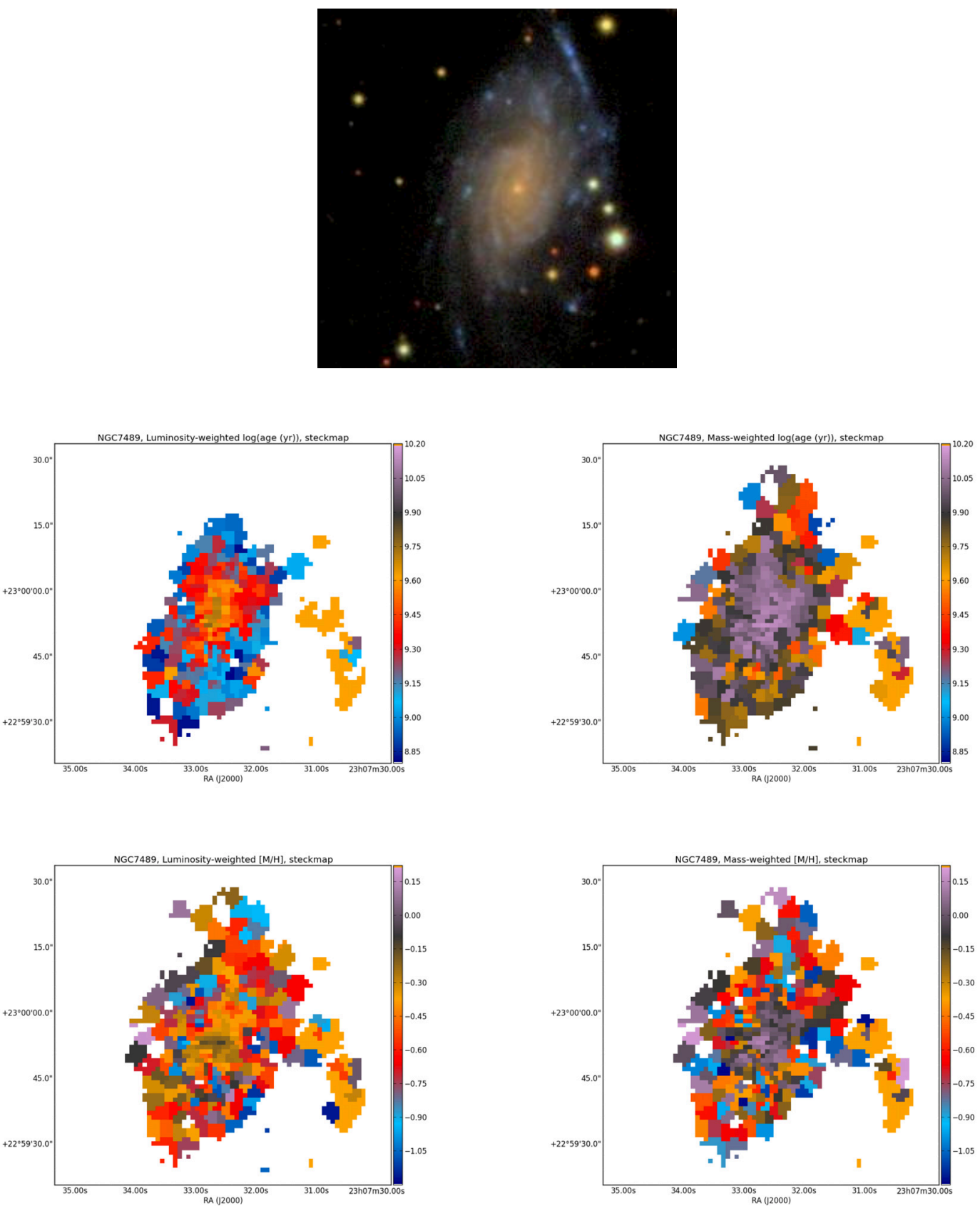

Fig. C.48. Mean log(age) and metallicity maps for NGC 7489. 
A\&A 570, A6 (2014)
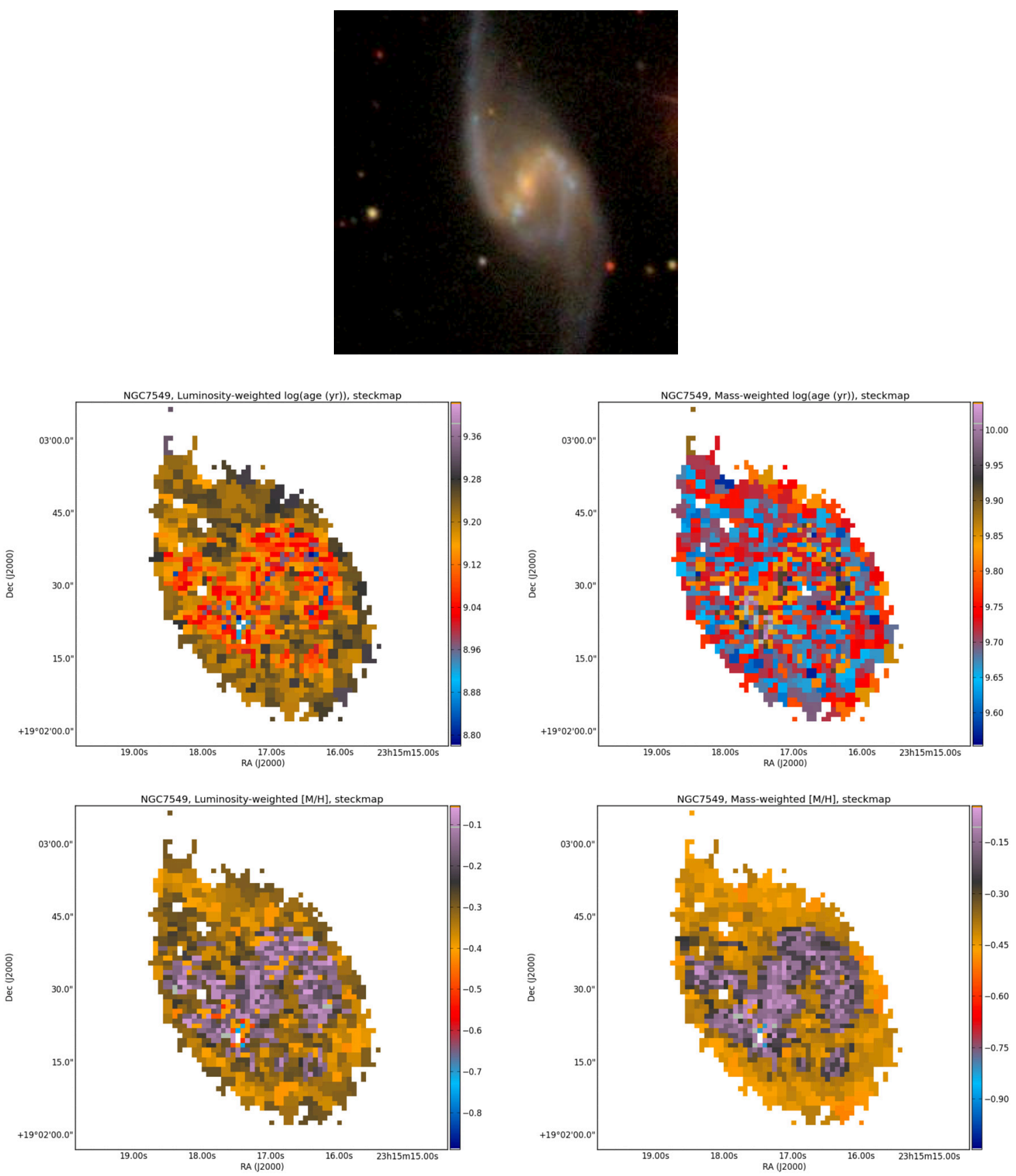

Fig. C.49. Mean log(age) and metallicity maps for NGC 7549. 
P. Sánchez-Blázquez et al.: Stellar populations in galaxy discs
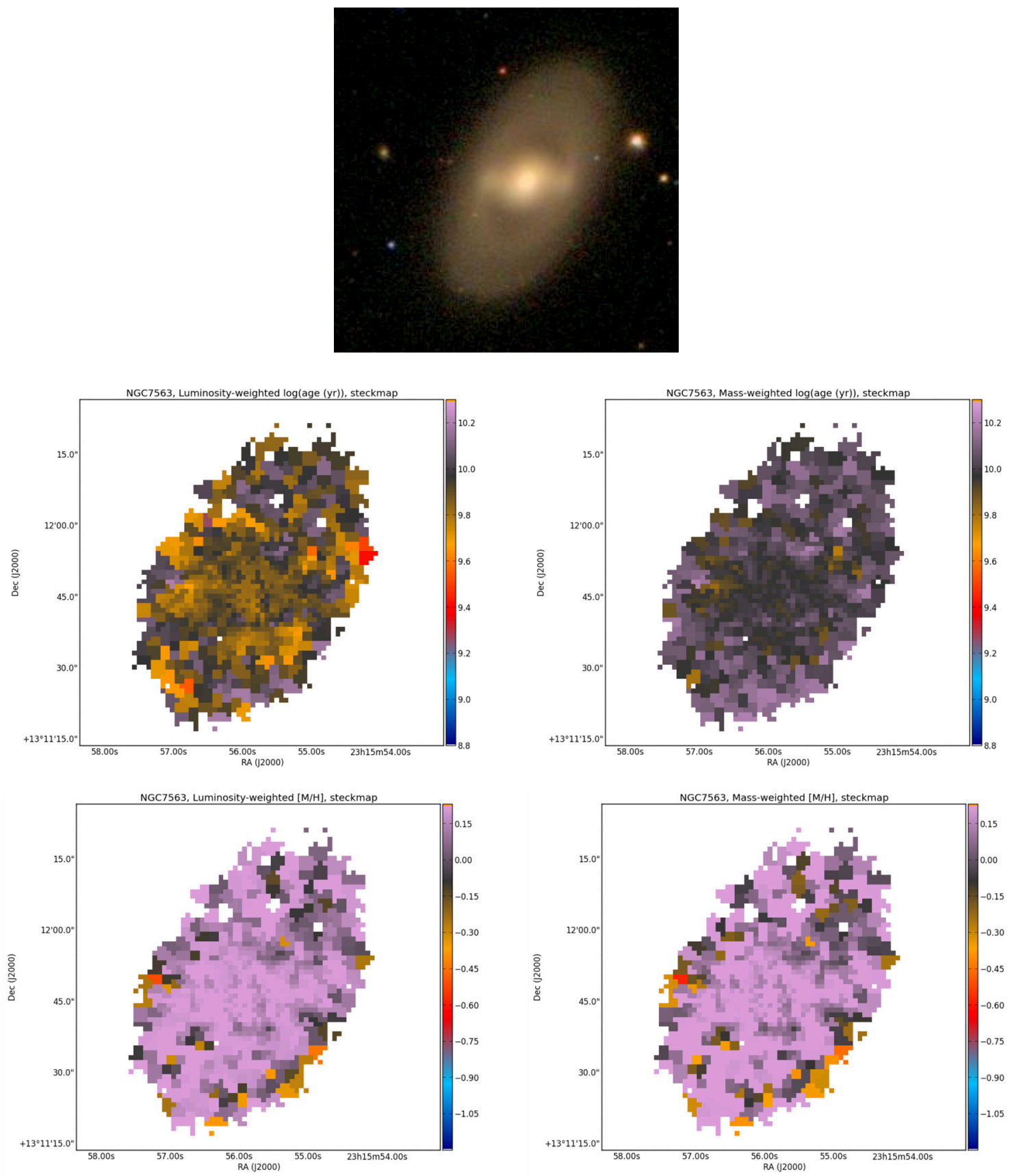

Fig. C.50. Mean log(age) and metallicity maps for NGC 7563. 
A\&A 570, A6 (2014)
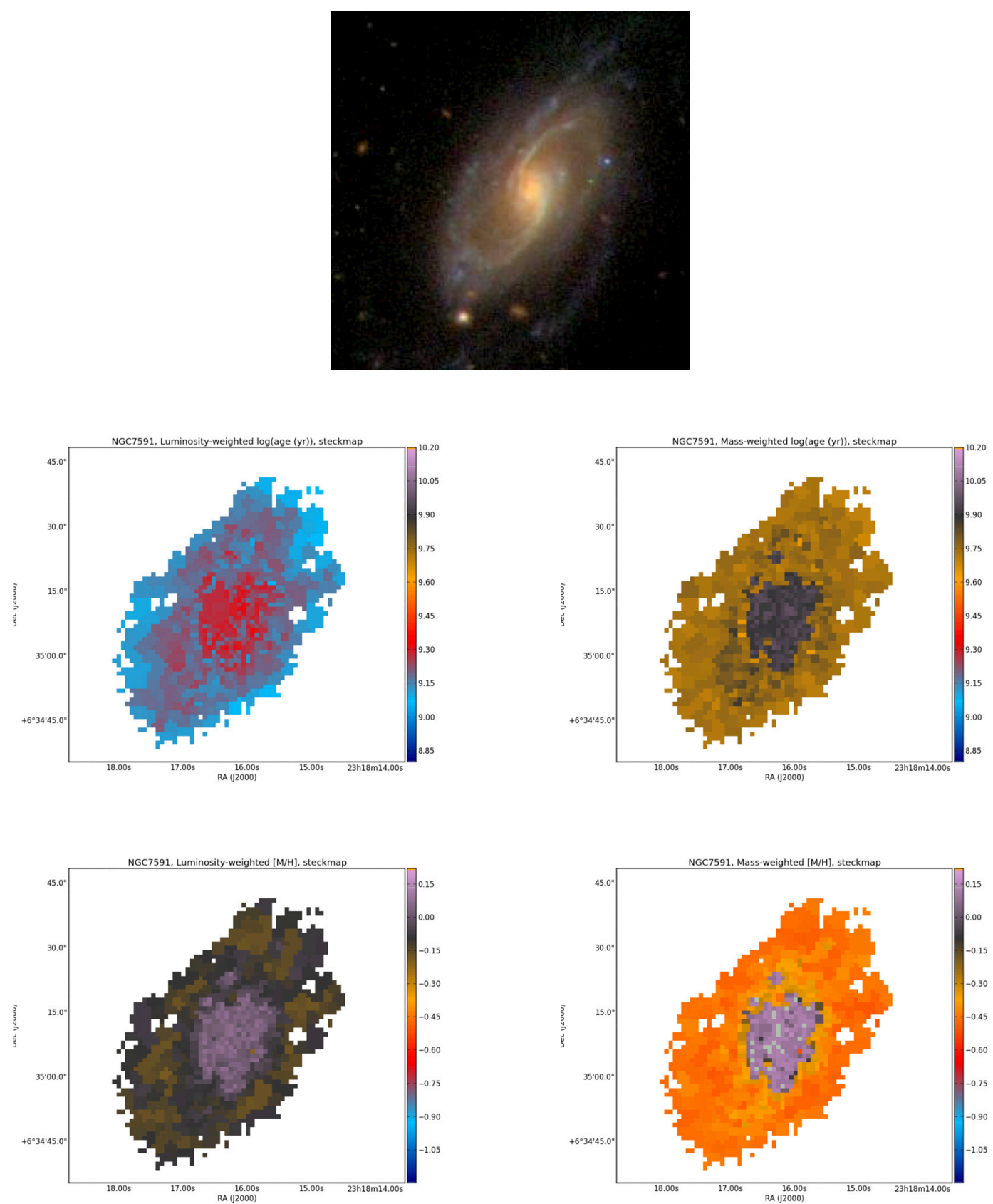

Fig. C.51. Mean log(age) and metallicity maps for NGC 7591. 
P. Sánchez-Blázquez et al.: Stellar populations in galaxy discs
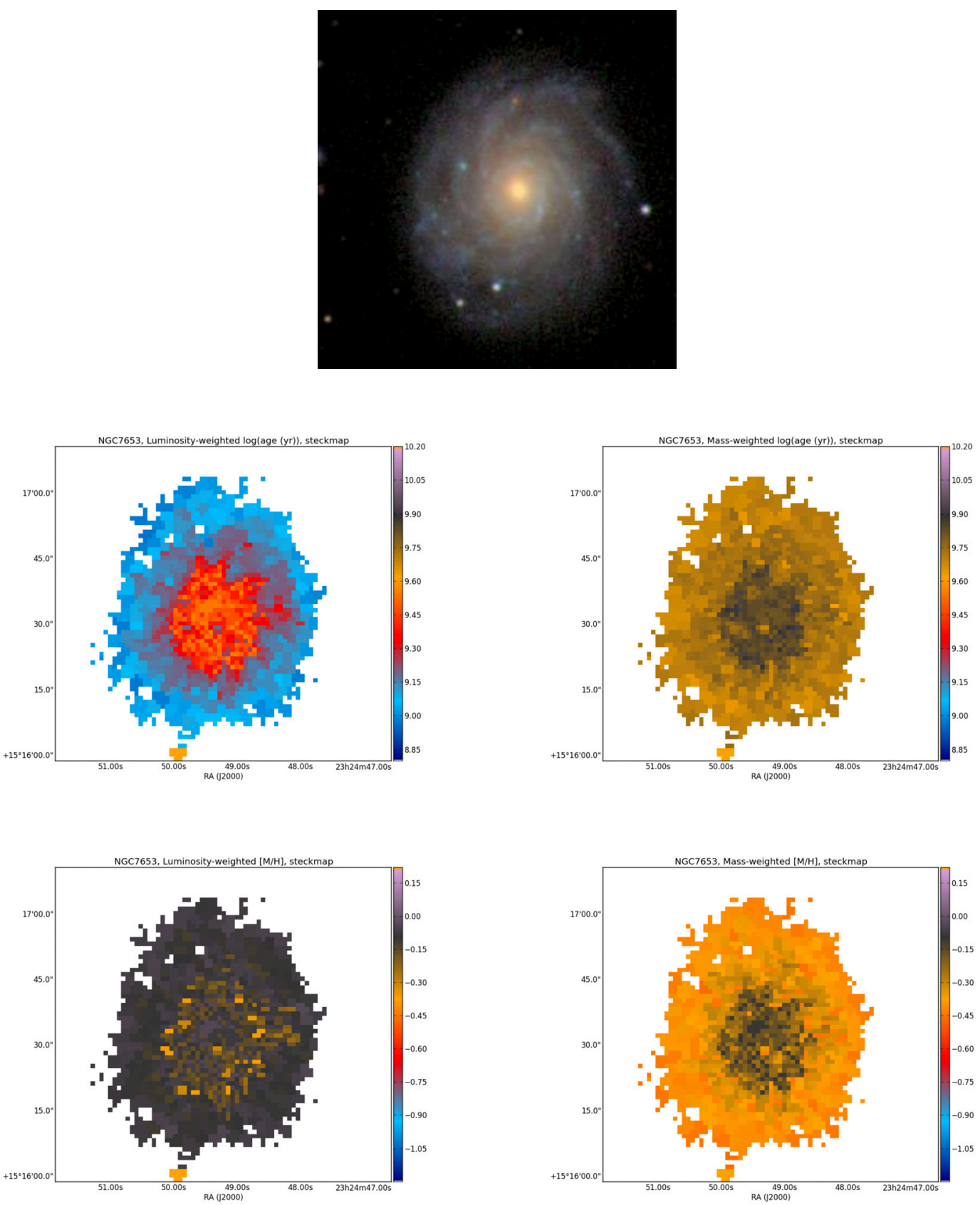

Fig. C.52. Mean log(age) and metallicity maps for NGC 7653. 

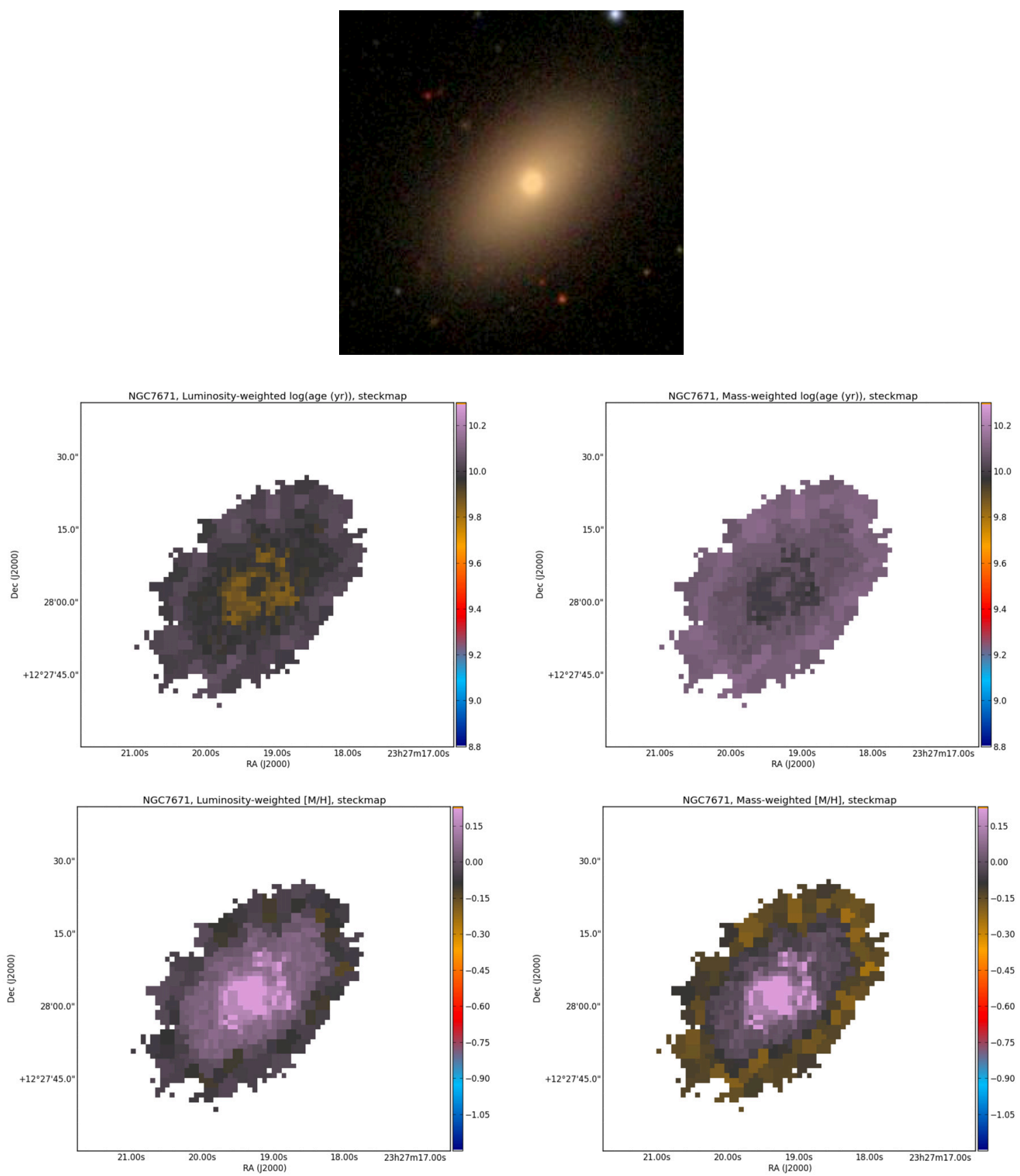

Fig. C.53. Mean $\log ($ age $)$ and metallicity maps for NGC 7671. 
P. Sánchez-Blázquez et al.: Stellar populations in galaxy discs
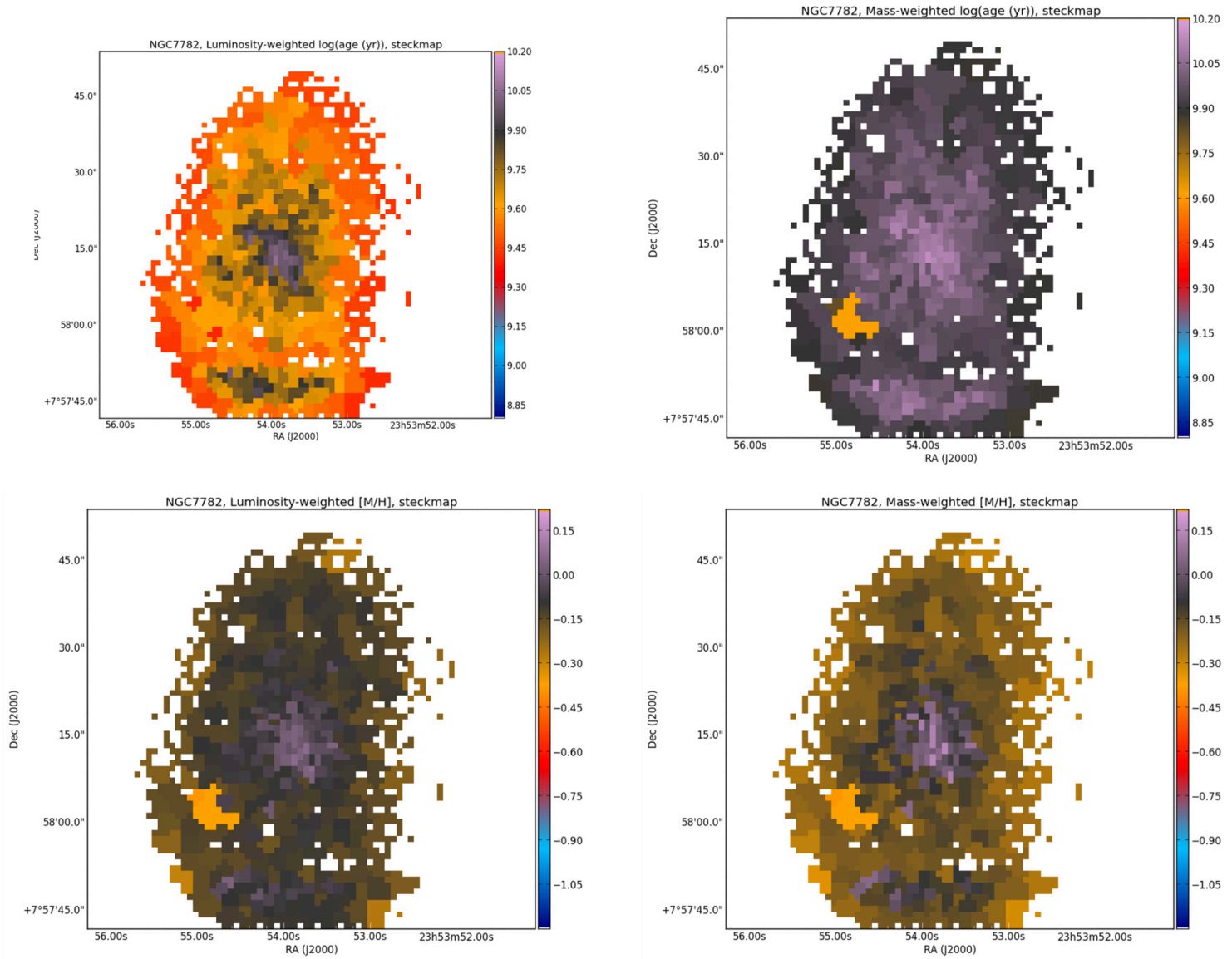

Fig. C.54. Mean log(age) and metallicity maps for NGC 7782. 

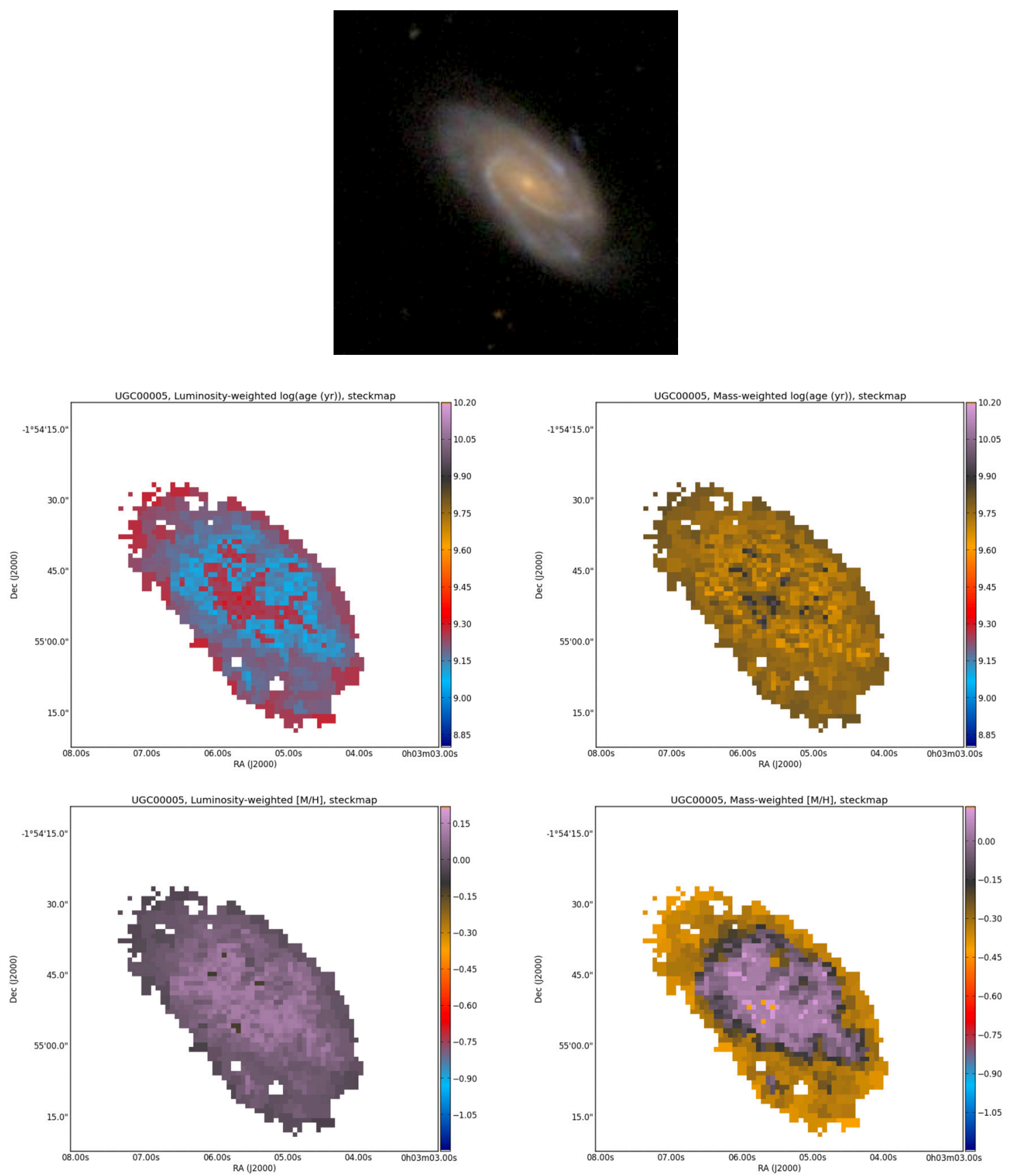

Fig. C.55. Mean log(age) and metallicity maps for UGC 00005. 
P. Sánchez-Blázquez et al.: Stellar populations in galaxy discs
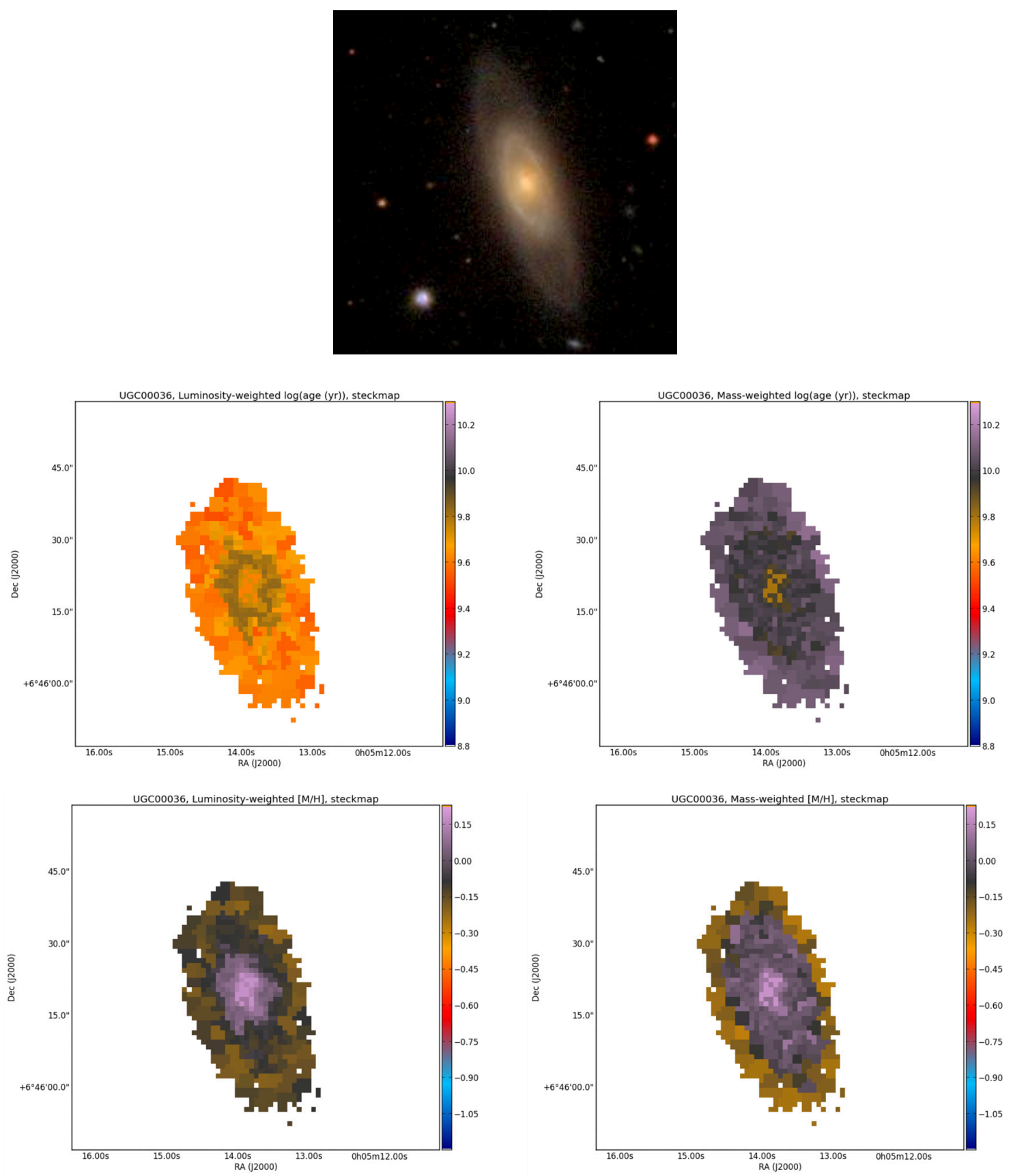

Fig. C.56. Mean $\log ($ age $)$ and metallicity maps for UGC 00036. 
A\&A 570, A6 (2014)
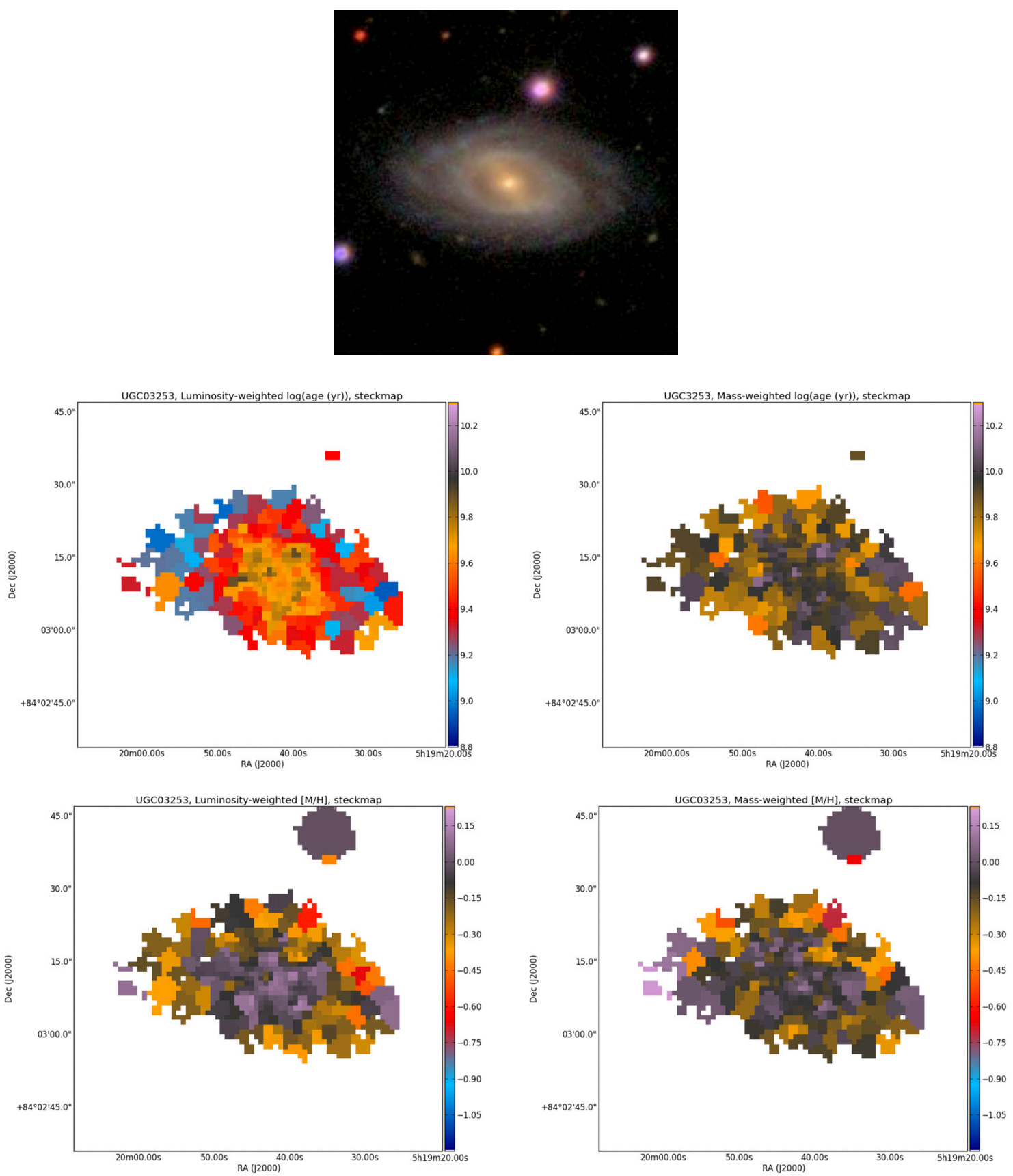

Fig. C.57. Mean log(age) and metallicity maps for UGC 03253 . 
P. Sánchez-Blázquez et al.: Stellar populations in galaxy discs
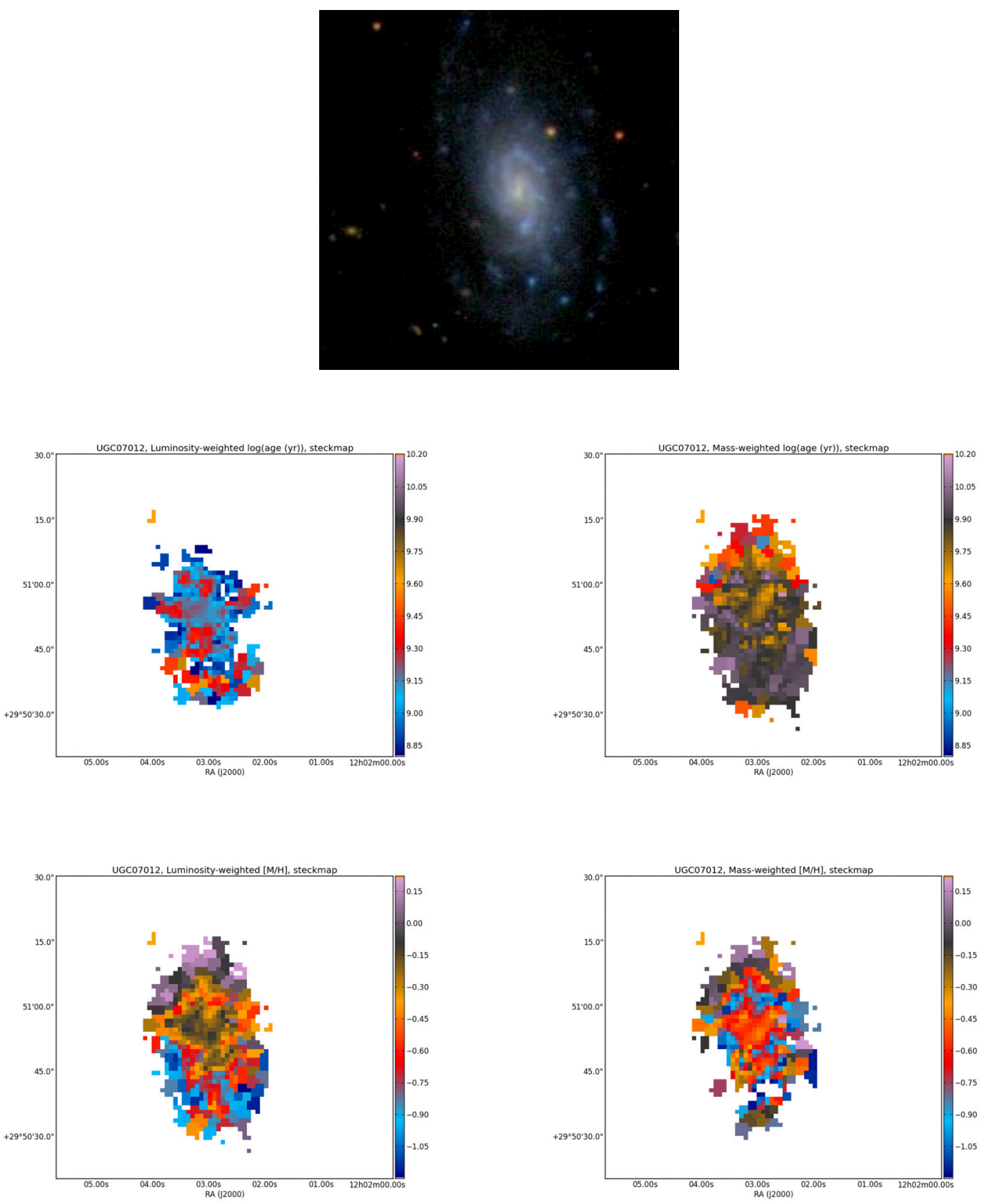

Fig. C.58. Mean log(age) and metallicity maps for UGC 07012. 
A\&A 570, A6 (2014)
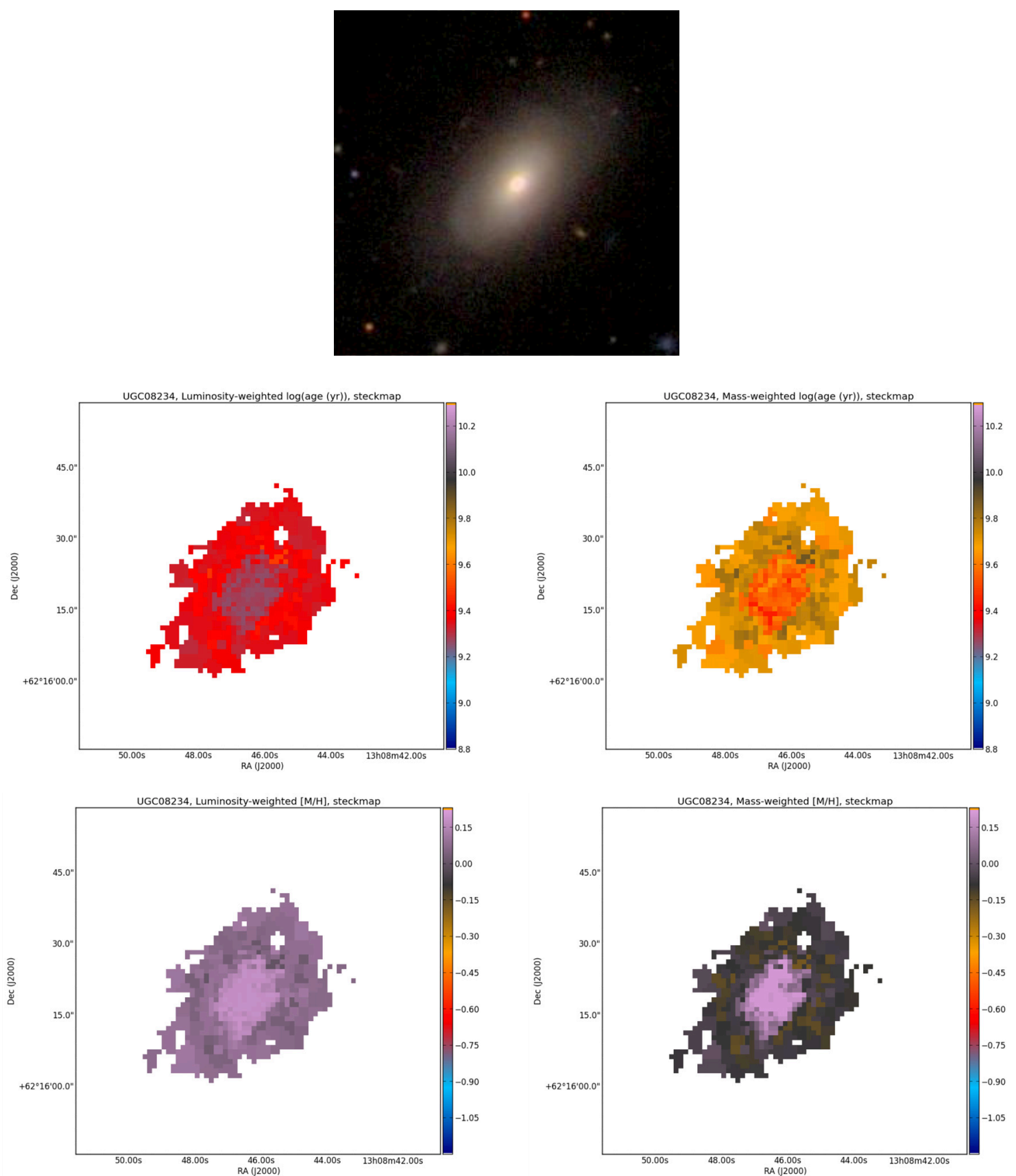

Fig. C.59. Mean log(age) and metallicity maps for UGC 08234. 
P. Sánchez-Blázquez et al.: Stellar populations in galaxy discs
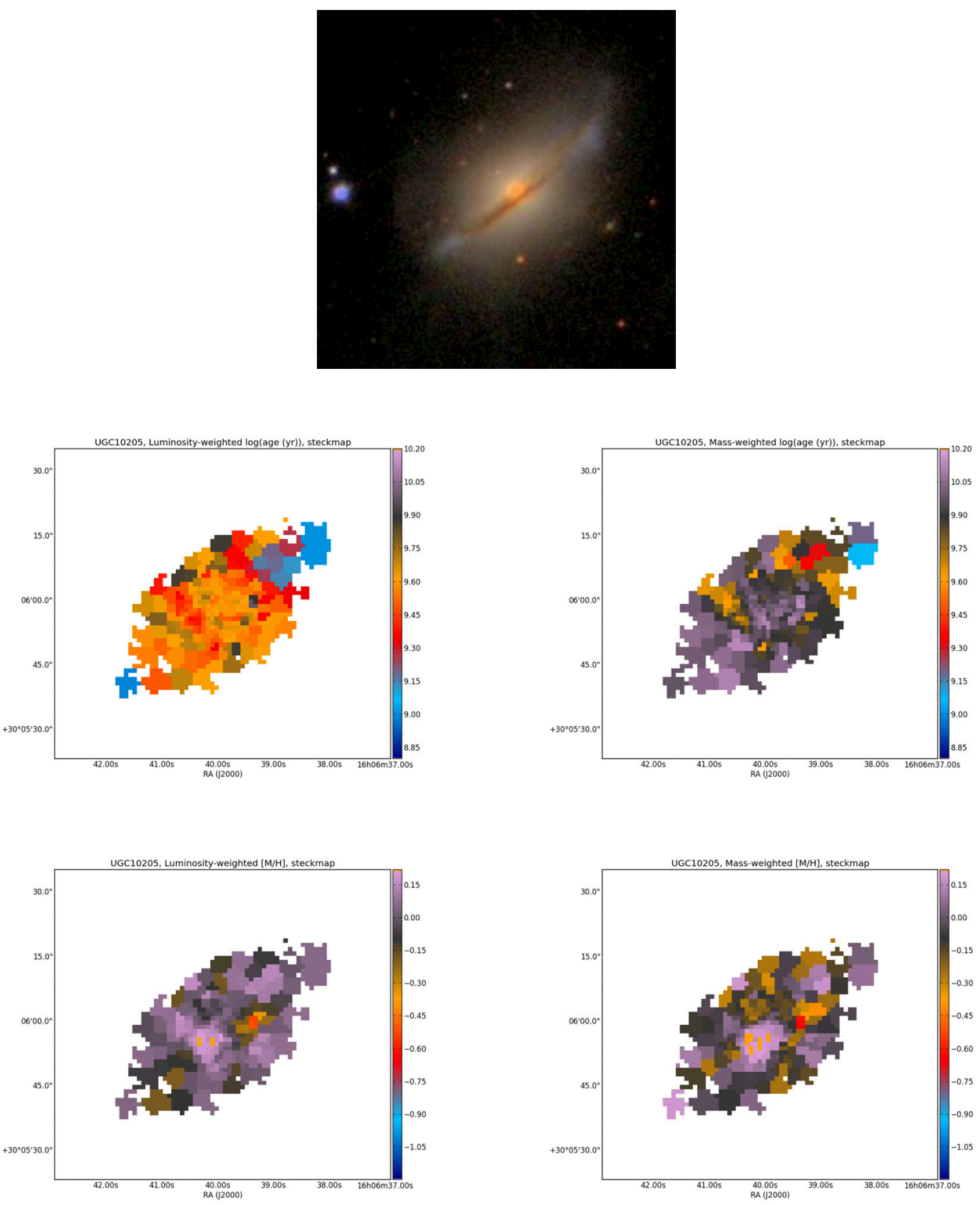

Fig. C.60. Mean log(age) and metallicity maps for UGC 10205. 
A\&A 570, A6 (2014)
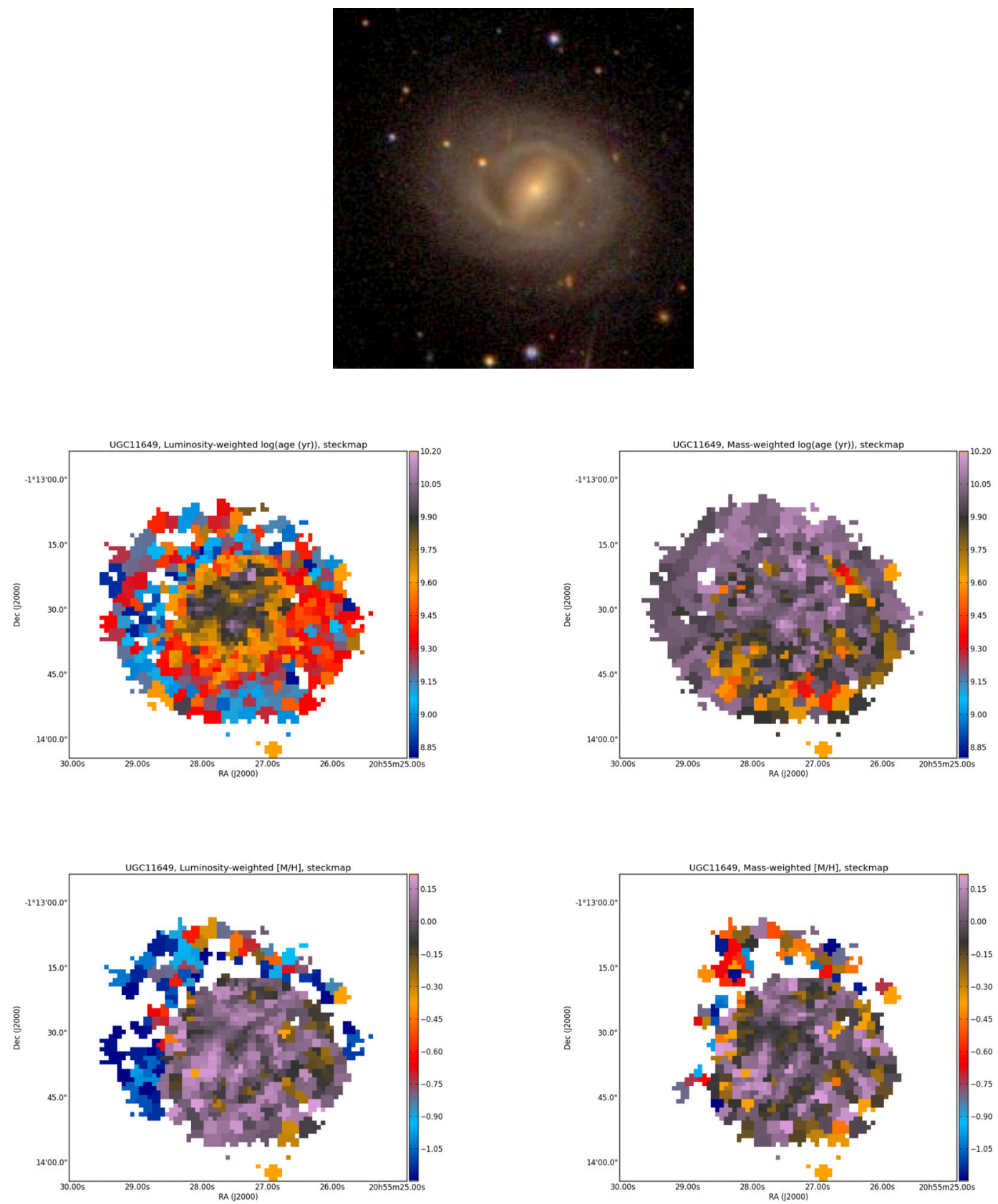

Fig. C.61. Mean log(age) and metallicity maps for UGC 11649. 
P. Sánchez-Blázquez et al.: Stellar populations in galaxy discs
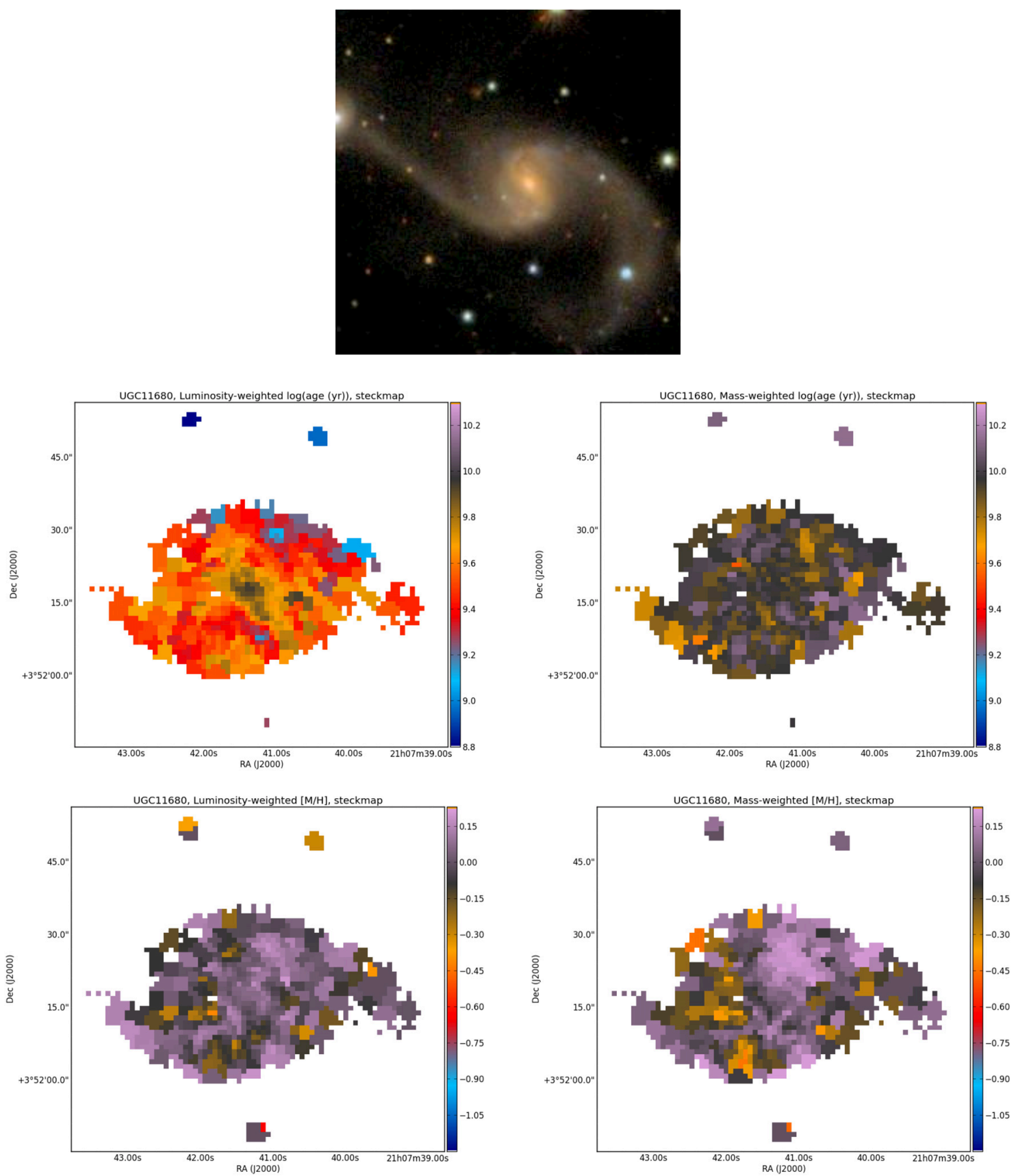

Fig. C.62. Mean log(age) and metallicity maps for UGC 11680. 

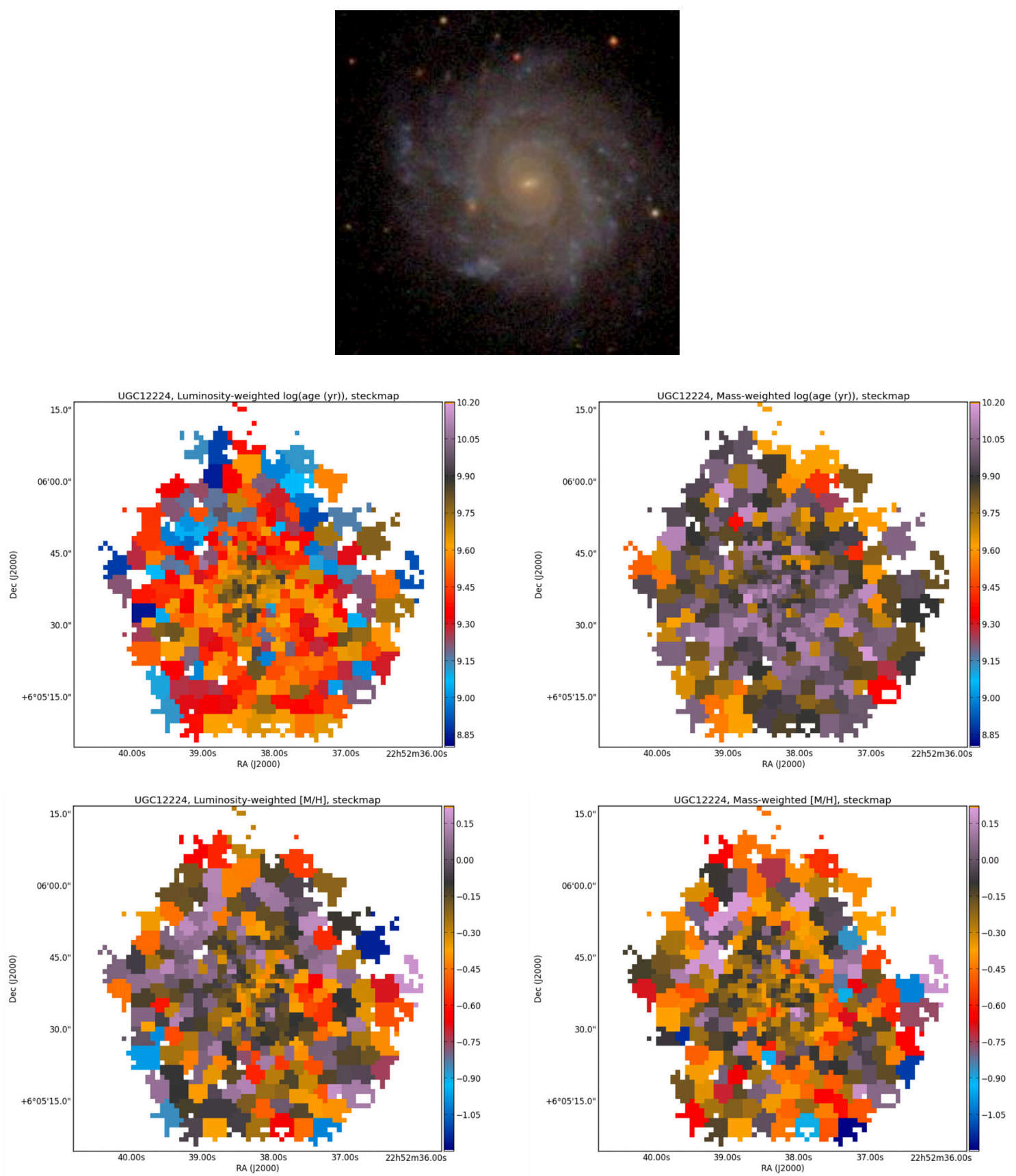

Fig. C.63. Mean log(age) and metallicity maps for UGC 12224 . 
P. Sánchez-Blázquez et al.: Stellar populations in galaxy discs
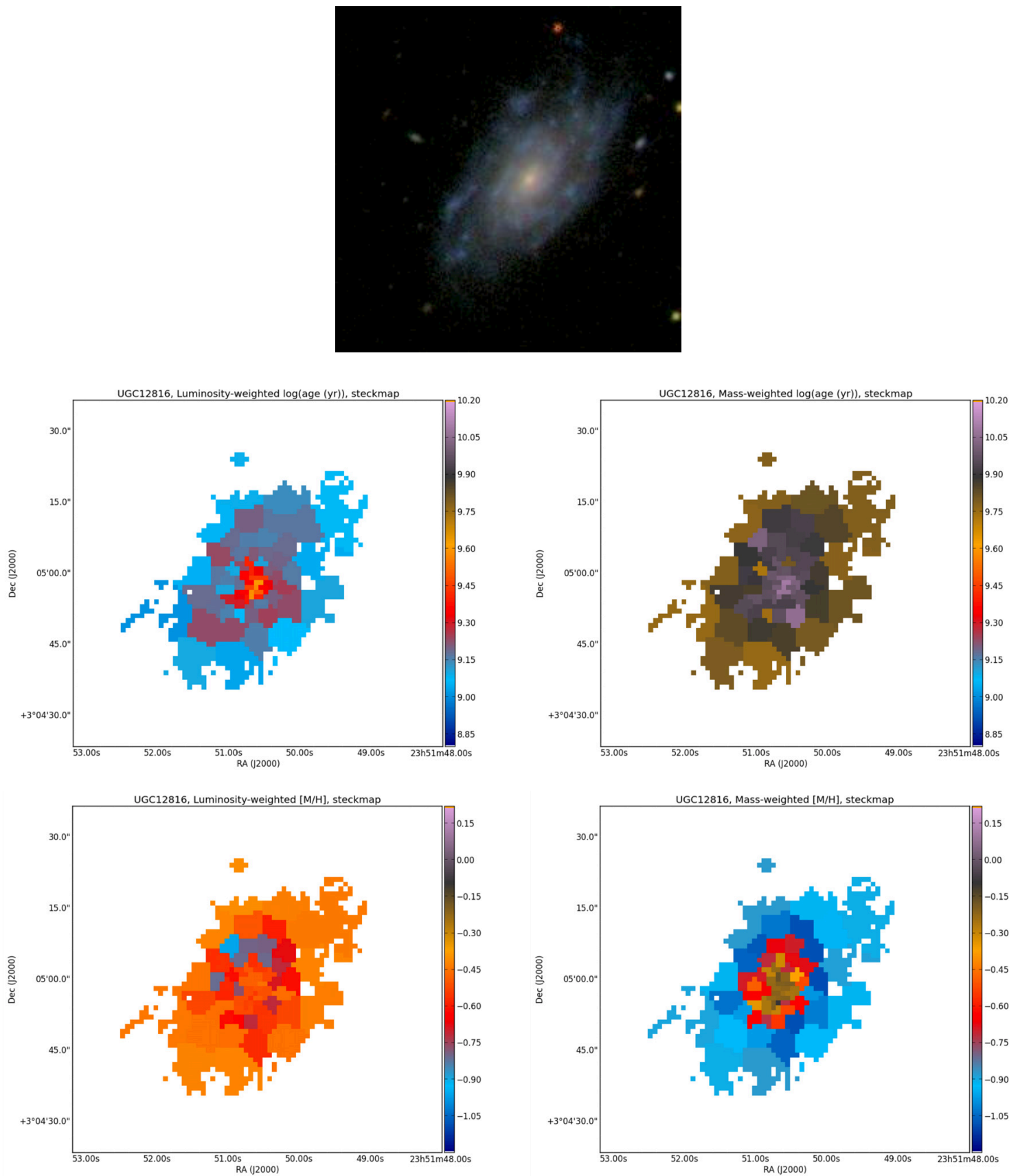

Fig. C.64. Mean log(age) and metallicity maps for UGC 12816. 